https://helda.helsinki.fi

\title{
The periodic table and the physics that drives it
}

\section{Schwerdtfeger, Peter}

2020-07

Schwerdtfeger , P , Smits , O R \& Pyykkö , P 2020 , ' The periodic table and the physics that drives it ' , Nature reviews chemistry , vol. 4 , no. 7 , pp. 359-380 . https://doi.org/10.1038/s41570-020-0195-y

http://hdl.handle.net/10138/323849

https://doi.org/10.1038/s41570-020-0195-y

unspecified

acceptedVersion

Downloaded from Helda, University of Helsinki institutional repository.

This is an electronic reprint of the original article.

This reprint may differ from the original in pagination and typographic detail.

Please cite the original version. 


\section{The Periodic Table and the Physics That Drives It}

Peter Schwerdtfeger ${ }^{1,2}$, Odile R. Smits ${ }^{1} \&$ Pekka Pyykkö $^{3}$

${ }^{1}$ Centre for Theoretical Chemistry and Physics, The New Zealand Institute for Advanced Study and the Institute for Natural and Mathematical Sciences, Massey University Auckland, 0632 Auckland, New Zealand,

${ }^{2}$ Centre for Advanced Study (CAS) at the Norwegian Academy of Science and Letters, Drammensveien 78, NO-0271 Oslo, Norway, and

${ }^{3}$ Department of Chemistry, University of Helsinki, P.O.B. 55 (A.I. Virtasen aukio 1), 00014 Helsinki, Finland,

March 5, 2020

Orcid IDs: P. Schwerdtfeger: https://orcid.org/0000-0003-4845-686X

O. Smits: http://orcid.org/0000-0003-1259-147X

P. Pyykkö: https://orcid.org/0000-0003-1395-8712 
Sentence for Table of Content. As the International Year of the Periodic table turned to an end in 2019, we reflect on the chemistry and physics that drives the Periodic table of the elements. This includes aspects of periodic trends, relativistic electronic structure theory, nuclear structure theory and the astrophysical origin of the elements. 
Mendeleev's introduction of the periodic table of elements is one of the most important milestones in the history of chemistry as it brought order into the known chemical and physical behaviour of the elements. The periodic table can be seen as parallel to the Standard Model in particle physics, in which the elementary particles known today can be ordered according to their intrinsic properties. The underlying fundamental theory to describe the interactions between particles comes from quantum theory, or more specifically from quantum field theory, and its inherent symmetries. In the periodic table, the elements are placed into a certain period and group based on electronic configurations that originate from the Pauli and Aufbau principles for the electrons surrounding a positively charged nucleus. This order enables us to approximately predict the chemical and physical properties of elements. Apparent anomalies can arise from relativistic effects, partial-screening phenomena (of type lanthanidecontraction), the compact size of the first shell of every l-value, ambiguities in electron configurations and the breakdown of assigning a dominant configuration owing to configuration mixing and dense spectra for the heaviest elements in the periodic table. For the short-lived transactinides, the nuclear stability becomes an important factor in chemical studies. Nuclear stability, decay rates, spectra and reaction cross sections are also important for predicting the astrophysical origin of the elements including the production of the heavy elements beyond iron in supernova explosions or neutron star mergers. In this Review we criti- 
cally analyse the periodic table of elements and the current status of theoretical predictions and origins for the heaviest elements, which combine both quantum chemistry and physics. 


\section{1 [H1] Introduction.}

In 1869 Dmitri Ivanovich Mendeleev ordered the known elements into what he termed the 'Periodic table of the elements' (PTE) on the basis of their increasing atomic weight and chemical similarity ${ }^{1}$. Mendeleev's PTE was proposed five years after Lothar Meyer had organised the 28 known elements into a table, of which six columns were labelled with valence number and five rows with atomic weight (Box 1 and for an historical account see Refs 2-8).

Mendeleev not only correctly identified several of the then unknown elements, such as Ge, Sc, Ga and Tc - that were subsequently discovered in 1876, 1879, 1886 and 1937, respectively — but also corrected some erroneous atomic weights such as for Be, In, Ce and U. An 1885 version of a wall-hanging PTE is shown in Fig.1a. Mendeleev had no knowledge of the internal structure of an atom or nucleus; a more detailed picture started to emerge only in 1911 with Ernest Rutherford's discovery of the atomic nucleus. The development of the PTE over the past 150 years is nicely illustrated at the Internet Database of Periodic Tables ${ }^{9}$ and a summary is provided in Fig.1. In the most recent version of the PTE (Fig.1c),

elements are ordered according to their atomic number $Z$ (the number of protons inside the nucleus), thus avoiding irregularities in mass numbers due to different numbers of neutrons inside the nucleus. As of today, 118 elements are experimentally known, with the most recent additions to the PTE being the main group elements from Nh $(Z=113)$ to $\mathrm{Og}(Z=118)$, thus successfully completing the 7th period of the PTE. 
The PTE is the most fundamental pillar of chemistry ${ }^{4}$ : molecules, large or small, are all made of interacting atoms from the PTE forming various types of chemical bonds. To cite Shaik and colleagues, "The periodic table gave rise to a central paradigm, which did for chemistry what Newton had done for physics and Darwin for biology"7. Questions naturally arise from this ordering system: what are the underlying (quantum) principles of the PTE? Where does the PTE end from an electronic or nuclear point of view? How far can we go in the synthesis of new elements and isotopes both in the laboratory and in the interstellar environment? Can we keep using the same approach to unambiguously place the elements with nuclear charge $Z>118$ into the PTE (as for example suggested in 2011 by one of the authors and shown in Fig.1c) $)^{10}$ ?

In this review, we address fundamental questions concerning the PTE and discuss the current status in this field from a quantum theoretical point of view ${ }^{11,12}$. We describe the underlying physical principles which guide the shape of the PTE including the elements up to a certain critical nuclear charge $(Z \approx 172)$. We focus on anomalies in chemical and physical properties rather than on similarities between the elements within a certain group. Furthermore, we discuss the astrophysical origin and nuclear stability of the elements including most recent developments in the field of nuclear structure theory. 


\section{2 [H1] From fundamental physics to the periodic table.}

The PTE is as fundamental to chemists as is the table of elementary particles to physicists (Fig.2). We all know that atoms interact to form chemical bonds (note that the term 'chemical bond' is a fuzzy concept, it does not strictly correspond to a quantum mechanical observable. It is a however useful concept derived from quantum theoretical principles ${ }^{13-15}$ and can be attributed to the lowering of the electronic kinetic energy, concomitant with the constructive interference between the constituents in the molecular wavefunction ${ }^{16,17}$ ). In much the same way, fermions (spin 1/2 particles, like the electron) interact through (gauge) fields described by the exchange of bosons (integer spin particles). Bosons are the carriers of the fundamental forces known in nature and are accurately described (as far as we know) by the standard model: the electromagnetic force is mediated by photons, the weak force (responsible for the $\beta$ decay in nuclei and the existence of the heavier elements in the PTE) is mediated by $W^{ \pm}$and $Z$ bosons, and the strong force (responsible for the existence of protons, neutrons and nuclei, in general) is mediated by gluons. The fourth fundamental interaction in nature, the gravitational force, has yet to be unified with the standard model, which represents one of the major challenges in physics. If the gravitational force can be quantized, the carriers of this force would also be bosons, so-called gravitons. All four fundamental forces are important for the astrophysical production and existence of the elements in the PTE and, ultimately, for the existence of life in our universe. Finally, the Higgs spin zero boson provides the mass for the particles in the standard model (except, perhaps, for the neutrinos). 
Why do we mention the fundamental principles of particle physics here? The answer is that the concept behind the PTE is strongly connected to fundamental physics involving not only atomic and molecular, but also particle and nuclear physics, two fundamental aspects that are usually not part of mainstream chemistry teaching and might not be familiar to most chemists. Starting with the electronic shell structure (the nuclear structure is discussed further below), the population of the PTE is governed by both the Pauli and the Aufbau principles. At a more fundamental level, the spin statistics theorem in physics (formulated by Fierz and Pauli ${ }^{18}$ ) demands that for fermions - such as the electron — the manyparticle wavefunction $\psi\left(\mathbf{r}_{i}, t\right)$ has to be antisymmetric with respect to the permutation of two particles, $i$ and $j$, from which the Pauli principle in a single-particle picture (mean-field theories such as Hartree-Fock or Kohn-Sham) follows. For chemists, this simply means admitting only one electron per single-particle state. This mean-field picture then leads to the famous Aufbau principle introduced by Bohr and Pauli that, together with Hund's rule, is considered as the second building block of the PTE, after the atomic number ordering.

Chemical behaviour is the third most important criterion for the elements in the PTE and an essential tool for all chemists. Similarities in the valence electron configurations for two atoms usually imply similar chemical properties, although subtle shell structure effects can lead to anomalies in the chemical and physical behaviour discussed below. The electron configuration of a multi-electron atom, or more precisely the configuration list including occupation numbers for individual one-electron states, is (together with the atomic number) an important parameter for placing an element into the PTE. The Schrödinger equation gives us 
the eigenfunctions in the form of complex many-electron wavefunctions and the corresponding eigenstates (that is, the spectrum) of an atom, molecule or a condensed phase. From the solutions of the Schrödinger equation we obtain physical properties (such as the dominant electron configuration), which give us important insights into the chemical behaviour of the elements. ${ }^{19}$ Together with thermodynamics and statistical physics, this differential equation lies at the very heart of chemistry.

From the solution of the stationary Schrödinger equation for a hydrogen-like atom we know that $\left(n l m_{l}\right)$ states with the same principal quantum number $n$ are energetically degenerate. In the relativistic case, this degeneracy is partially lifted owing to spin-orbit coupling, which can become very large for heavy elements, leading to the $l>0$ levels split into levels of $j=l \pm 1 / 2$. Quantum electrodynamics (QED) further lifts the degeneracy between the $s$ and $p_{1 / 2}$ levels by a small amount. This so-called Lamb shift is tiny, $4.372 \times 10^{-6} \mathrm{eV}$ for the $2 s-2 p_{1 / 2}$ splitting in the hydrogen atom, but can approach chemical relevance for heavy elements, ${ }^{20,21}$ such as $\mathrm{Au}(0.019 \mathrm{eV}$ for the $6 \mathrm{~s}$ level $)$ or $\mathrm{Og}\left(0.016 \mathrm{eV}\right.$ for the $7 p_{3 / 2}$ level $) .{ }^{22}$

Degeneracies are further broken in the screened Coulomb potential of multi-electron atoms, for example, following the Aufbau principle, the $2 s$ levels are filled before the $2 p$ levels. J. C. Slater was the first to extend systematically the one-particle solutions of the Schrödinger equation to a multi-electron system ${ }^{23}$ following earlier work by Zener ${ }^{24}$. In the so-called mean-field model for a multi-electron atom, each electron is moving in the field generated by all other electrons and the nucleus experiencing a reduced nuclear charge, $Z^{\text {eff }}=Z-\sigma$, due 
to the shielding (or screening) by all the other electrons expressed by the screening constant $\sigma$. Slater's rules provide numerical values for $\sigma$ in multi-electron systems that enable the approximate calculation of the total electronic energy. The idea of a screening effect leads to the lifting of degeneracies and explains why the $4 s$ level is occupied before the $3 d$ levels (for example, in the case of $\mathrm{K}, Z^{\mathrm{eff}}=2.20$ for $4 s^{1}$ and $Z^{\mathrm{eff}}=1.00$ for $3 d^{1}$ valence configuration). Slater's approach can be seen as the first successful quantum theoretical attempt to place the elements correctly into the PTE using the Aufbau principle. It places the electrons obeying the Pauli principle into the levels experiencing the highest effective nuclear charge first. However, if the gap between two one-electron levels is smaller than the exchange energy correction, the lowest energy is obtained for the high-spin configuration. Despite its early success, the original Slater rules have their limitations. They did not explain why the $2 s$ level is occupied before the $2 p$ level because these share the same screening factor $\sigma$, or more subtle differences in electron configurations as found, for example, in the group 10 elements: $\mathrm{Ni}\left(3 d^{8} 4 s^{2}\right), \mathrm{Pd}\left(4 d^{10}\right)$, Pt $\left(5 d^{9} 6 s^{1}\right)$, and Ds $\left(6 d^{8} 7 s^{2}\right)$. It is, however, not important which screened-Coulomb potential is chosen for the Aufbau discussion ${ }^{8}$. For example, in the past, the Thomas-Fermi model was used to determine the atomic number at which $l$-electrons for a given $l_{\max }$ first appear. ${ }^{25-28}$

A far more accurate determination of electron configurations is achieved using meanfield methods such as relativistic Hartree-Fock or Kohn-Sham density functional theory (KS-DFT). With these methods one can easily obtain low-lying electronic states associated with dominant electron configurations and effective nuclear charges for a specific electronic 
shell. For example, Hartree defines the screening constant as $\sigma_{n l}=Z-\langle r\rangle_{n l}^{\mathrm{H}} /\langle r\rangle_{n l}$ for a specific nucleus of charge $Z$ and shell $(n l)$, where $\langle r\rangle_{n l}^{\mathrm{H}}$ is the unscreened hydrogenic value, which can be obtained analytically (for the relativistic case, we simply extend it to the shell with quantum numbers $(n l j)) .{ }^{29}$ From relativistic Hartree-Fock calculations of Li we obtain $Z_{2 s}^{\mathrm{eff}}=1.55$ and $Z_{2 p}^{\mathrm{eff}}=1.04$, and for $\mathrm{K}$ we obtain $Z_{4 s}^{\mathrm{eff}}=4.59$ and $Z_{3 d}^{\mathrm{eff}}=1.10$, which are qualitatively correct. Even if we use post Hartree-Fock theory and include electron correlation, for example by a configuration interaction (CI) treatment that accounts for the mixing of configuration states, we can still determine the dominant configurations and try to assign an approximate electron configuration to an atom. This simplified picture can however break down if the energy levels are not well separated and the spectrum becomes dense owing to quasi-degeneracies - ultimately, the correct ground state can only be determined by solving the Schrödinger equation or more precisely, the Dirac equation that includes important QED effects (Box 2). Let us look again at the electron configurations in the group 10 elements. The three dominant configurations - $\left[(n-1) d^{8} n s^{2}\right],\left[(n-1) d^{9} n s^{1}\right]$ and $\left[(n-1) d^{10}\right]-$ lead to a total of 13 states separated by small energy differences as shown in Fig.3a. However, this does not prevent us from placing these elements correctly into group 10 of the PTE.

Moving to group 11, of the three $n s^{1}$ coinage-metal atoms $(\mathrm{Cu}, \mathrm{Ag}, \mathrm{Au})$, only one behaves chemically as expected' and that is silver ${ }^{30} . \mathrm{Cu}$ is anomalous having a radially nodeless, and therefore compact, $3 d$ shell. Au is anomalous for both its lanthanide contraction owing to the filling of the $4 f^{14}$ shell and (even more so) strong relativistic effects. Ag in between has a $d$-shell node and only moderate relativistic effects. Rg even changes its 
ground state to $d^{9} s^{2}$ due to a strong relativistic $7 s$ stabilization.

Despite the theoretical limitations of using electron configurations because of configuration mixing, we can safely place all known 118 elements into the PTE using the Aufbau principle (Edward G. Mazurs presented a periodic table in 1955 classified later as Subtype IIIC3-6b in which he correctly placed the elements into the PTE up to $Z=120^{31,32}$ ). As a matter of interest, the Aufbau principle follows the empirical rule proposed by Madelung in $1926^{33}$ and Janet in $1930^{34,35}$ as shown in Fig.3b, and used early on by Sommerfeld ${ }^{36}$ to fill the electronic configurations up to Rn. The Madelung-Janet (or diagonal) rule states that when considering consecutive neutral atoms, electrons are assigned to one-particle levels in order of increasing $(n+l)$ value, and for states of equal $(n+l)$, the electrons are assigned first to the lower $n$ value. For a detailed account on the Madelung-Janet rule see Ref. 37 . This rule is remarkably well fulfilled throughout the PTE, with only few exceptions in the superheavy element region because of strong relativistic effects. Nevertheless, the putative placement of the superheavy elements beyond $Z=120$ by one of the authors ${ }^{10}$ requires further investigations to correctly predict the electronic ground state and associated dominant configurations together with their chemical similarities to their lighter congeners - a major challenge for modern quantum chemistry. For example, Nefedov and co-workers performed multiconfiguration Dirac-Fock calculations for the superheavy elements with $Z=119-164$ (Ref.38). They showed that a major single configuration can still be assigned for these elements despite the fact that the $5 g, 6 f, 7 d$ and $8 p$ levels become close in energy. They further predict the $5 g$ occupation to start at $Z=125$. 
Despite the huge success of the Madelung-Janet rule, the most appropriate definition of the start and end points of the lanthanide and actinide series remains a matter of dispute $^{39,40}$. Inserting the lanthanides $\mathrm{La}-\mathrm{Yb}$ and actinides Ac-No between group 2 and 3, and $\operatorname{Lu}\left[4 f^{14} 5 d^{1} 6 s^{2}\right]$ and $\operatorname{Lr}\left[5 f^{14} 7 p^{1} 7 s^{2}\right]$ (note the difference in the occupation of $p$ and $d$ levels between the two elements) into group 3 fulfils the Madelung-Janet rule and results in a more natural placement of these elements into the PTE. However, placing La $\left[5 d^{1} 6 s^{2}\right]$ and Ac $\left[6 d^{1} 7 s^{2}\right]$ into group 3 and the series Ce-Lu and Th-Lr afterwards has the advantage to keep La and Ac as the first elements of the lanthanide and actinide series to which they give their names. In a set of molecules, $\mathrm{Xu}$ and Pyykkö ${ }^{41}$ find that $\mathrm{Lu}$ and $\mathrm{Lr}$ behave in a very similar way. Note, moreover, that the placement of the $4 f$-to- $6 f$ and the $5 g$ elements in Fig. 1c keeps the group number, $G$, equal to the number of valence electrons. We are not delving further into discussions of chemical similarities between the two different definitions of the group 3 elements as there are many different opinions on this. ${ }^{40,42}$ IUPAC conveniently avoids this controversy by leaving the two positions in periods 6 and 7 of group 3 empty and listing 15 instead of 14 elements for the lanthanides and actinides, thus counting from $f^{0}$ to $f^{14}$. Fuzzy concepts like chemical similarities or electron configurations do have their limits, but in our opinion do not reduce the importance of the PTE.

\section{$3 \quad[\mathrm{H} 1]$ Periodic trends and relativistic effects}

It has become (almost) a doctrine in chemistry that elements placed in the same group of the PTE show similar chemical and physical behaviour. Similarities and differences are 
based on the comparison of measurable quantities, either through experimental observables or through their calculations using the corresponding self-adjoint operators, if necessary to the highest accuracy available within a certain theory. A detailed discussion on the numerous similarities observed is beyond the scope of this Review, for recent accounts see Ref.43 and Ref. 44, and for main group elements see Ref. 19. Instead, we focus on some irregularities and anomalies within the PTE arising from shell structures and relativistic effects.

[H2] $1 s$ elements. We start our discussion by mentioning the two most abundant elements in our universe, $\mathrm{H}$ and $\mathrm{He}$, synthesized directly in the primordial nucleosynthesis roughly 10 seconds to 20 minutes after the Big Bang ${ }^{45}$. These are placed into group 1 and 18, respectively, although their chemical and physical behaviour is quite distinct compared to their heavier homologues in the PTE. Hydrogen is quite unique: from hydrogen we get biomolecules and water important for life on our planet. Watson-Crick pairing is a prime example in which hydrogen-bonding is required for the stability of DNA. Or think of the structure of benzene and its associated rich aromatic chemistry and compare it to $\mathrm{C}_{6} \mathrm{Li}_{6}$, which consists of $\mathrm{C}_{2}^{2-}$ fragments strongly aggregated through Li bridges ${ }^{46}$. A recent addition is the discovery of superconductors in hydrogen-rich phases ${ }^{47-49}$ and the search for metallic, high-pressure phases of pure hydrogen important for understanding the physics of large sized planets such as Jupiter. ${ }^{50}$

Even so He is the most abundant element in our universe, its chemistry is very limited. Needless to say that the electron configuration of He is $\left[1 s^{2}\right]$ - with the highest ionization 
potential of any element $(24.587387 \mathrm{eV})^{51}$ — whilst for the other noble gases it is $\left[n p^{6}\right]$. Both isotopes ${ }^{3,4} \mathrm{He}$ at low temperatures exhibit a distinct quantum nature and special phases in the bulk. Because of its chemical inertness, helium fits rather into group 18 than into group 2 of the PTE, although we note the existence of gas-phase cations, such as $\mathrm{HeH}^{+}$or metal helides, such as $\mathrm{VHe}^{3+}, \mathrm{YHe}^{3+}$ or $\mathrm{AlHe}^{3+}, 2^{52-54}$ and the observed high-pressure electride compound $\mathrm{Na}_{2} \mathrm{He}^{55}$. This is a prime example in which chemical similarity wins over electron configuration.

Although $\mathrm{H}$ and He clearly separate from the rest of the PTE, almost every chemist agrees that we can leave these elements in their current place in the PTE keeping their distinctive quantum nature in mind.

[H2] Primogenic shell effect. Continuing with trends within a group of the PTE, we note the compact size of the shells with every first-appearing $l$-value (termed primogenic ${ }^{56}$ or kainosymmetric effect, the special nature of which was pointed out by Shchukarev in 1971. ${ }^{57,58}$ The primogenic effect has far-reaching consequences, for example, it explains the existence of $\mathrm{P}_{4}$ and not of $\mathrm{N}_{4}{ }^{59}$ or, more importantly, the quite distinct chemistry of $\mathrm{C}$ versus $\mathrm{Si}^{60}$ and why life based on $\mathrm{Si}$ instead of $\mathrm{C}$ may not exist. This effect can be clearly understood by comparing the valence shell radii (in $\AA$ ) and screening constants $\sigma$ of $\mathrm{C}$ and Si (obtained from Hartree-Fock calculations): $\langle r\rangle_{2 s}^{\mathrm{C}}=0.839 \AA, \sigma_{2 s}^{\mathrm{C}}=2.22,\langle r\rangle_{2 p}^{\mathrm{C}}=0.921 \AA$, $\sigma_{2 p}^{\mathrm{C}}=3.13 ;\langle r\rangle_{3 s}^{\mathrm{Si}}=1.164 \AA, \sigma_{3 s}^{\mathrm{Si}}=7.86,\langle r\rangle_{3 p}^{\mathrm{Si}}=1.473 \AA, \sigma_{3 p}^{\mathrm{Si}}=9.51$. Similarly, the first-row transition elements have a compact $3 d$ shell and often their chemical behaviour differ to 
that of the heavier congeners. For example, $\mathrm{RuO}_{4}, \mathrm{OsO}_{4}$ and even $\mathrm{HsO}_{4}$ are experimentally known ${ }^{61}$, but $\mathrm{FeO}_{4}$ is not (in its reduced state $\left[\mathrm{FeO}_{4}\right]^{2-}$ and $\left[\mathrm{FeO}_{4}\right]^{-}$are $\mathrm{known}^{62}$ ). Trends down a group are further influenced by relativistic effects, which can change completely the chemical behaviour of the heavier elements.

[H2] Filling $l$-shells along a row of the PTE. Turning to periodic trends along a specific row, $66.7 \%$ of the $d$-block elements (not including the transactinides) and $78 \%$ of the $f$ block elements have $n d^{G-2}(n+1) s^{2}$ and $(n-1) f^{G-2}(n+1) s^{2}$ electronic configurations, respectively, where $G$ denotes the group number in the PTE. Some $d$-block elements prefer a greater $d$-population and some $f$-block elements prefer a single electron in the $d$-shell ${ }^{63}$. Nevertheless, different electron configurations lie close by in energy and we can successively fill the $d$-shell or $f$-shell using Hund's rule.

Allen and co-workers analyzed the $(n-1) d$ and $n s$ orbital energies for the transition elements $^{64}$. As the energies depend on the different $n s$ populations (Fig.3a), the concept of configuration energies $\epsilon_{\mathrm{CE}}$ previously introduced by Allen ${ }^{65}$ was used. Configuration energies for the transition elements can be defined as $\epsilon_{\mathrm{CE}}=-\left(p \epsilon_{s}+q \epsilon_{d}\right) /(p+q)$, where $p$ and $q$ are fractional occupation numbers for the $s$ and $d$ shells with orbital energies $\epsilon_{s}$ and $\epsilon_{d}$. This approach considers configuration mixing in this orbital space and avoids anomalies in trends arising from changes in configurations along the transition-metal row. Fig.3c,d show the results of these calculations and reveal some interesting trends.

We observe that the $(n+1) s$ shell comes at higher energy compared to the underlying $n d$ 
shell and the valence $s$ electrons are therefore more easily accessible. Further, the $s-d$ energy gap increases across a row, with a more prevalent increase for the first-row $(3 d)$ transition elements. The easy access of the $s$ valence shell is seen as the main reason why all transition elements, except $\mathrm{Sc}$, form divalent chlorides, $\mathrm{MCl}_{2}$. However, the chemistry of the transition metals is far more complicated because the $d$ subshell can contribute substantially to chemical bonding, especially for metals in higher oxidation states, even for the late transition elements as in the case of $\mathrm{CuF}_{4}^{-} \cdot{ }^{66}$ For the third row transition elements $(5 d)$ - and also for 4 th row (6d) transactinides, the relativistic $s$-shell contraction becomes large leading to an additional nuclear screening for the underlying $d$ shell. This effect becomes particularly large for the group 11 and 12 elements as clearly seen in Fig.3c,d, leading to well known chemical and physical anomalies (see discussion below). Finally, for the $3 d$ transition metals we see a steep increase in configuration energies up to the element with half-filled $d$ shell, Mn, defining a threshold between the early and late transition metals (for a more detailed discussion on this subject see Refs.58,67).

[H2]Relativity and the PTE. The Schrödinger equation, which has served the chemistry community extremely well for the past 50 years, emerges as the non-relativistic limit (velocity of light $c \rightarrow \infty$ ) from its relativistic extension, the Dirac equation (Box 2). The electronic Dirac equation did not come so easy to the discrete-basis quantum chemistry community because of the appearance of a negative-energy continuum, the Dirac sea. This additional feature causes trouble in the treatment of elements with very high nuclear charge, $Z \alpha_{\mathrm{EM}}>1$, with $\alpha_{\mathrm{EM}}$ being the fine-structure (or electromagnetic coupling) constant defined as $1 / \alpha_{\mathrm{EM}}=$ 
$4 \pi \epsilon_{0} \hbar c / e^{2}=137.035999084(21){ }^{68}$ Nevertheless, the enormous progress in this field over the past four decades enabled us to deal efficiently with the electronic Dirac equation and to obtain very accurate solutions ${ }^{69}$ (Box 3$)$.

Over the past few decades we learned that relativistic effects are more important than originally thought. Valence electrons move slowly compared to the velocity of light when they are far from the nucleus but fast when they are near the nucleus, especially for the heavy elements with high nuclear charge ${ }^{70-73}$. A closer analysis shows that direct relativistic effects arise from the innermost region of the radial wavefunction (mainly from the K-shell (1s) range and, to a smaller extent, the L-shell range for all $s$-orbitals) as well as from the spin-orbit splitting of all $n p$-orbitals ${ }^{74,75}$. Indirect relativistic contributions arise from the relativistic changes of the other orbitals. Both direct and indirect relativistic effects are illustrated in Figure 4a,b.

Because of the direct relativistic effects, the nucleus becomes more screened especially by the $s$ and $p_{1 / 2}$ electrons that have a substantial electron density near the nucleus, thus lowering $Z^{\text {eff }}$. As a consequence, the more diffuse orbitals with higher angular momentum feel a weaker nuclear attraction that results in their expansion and destabilization. An early example for the destabilization of the $5 d$ shell of the $\mathrm{Hg}$ atom was reported by Mayers ${ }^{76}$ in 1957. In addition, spin-orbit coupling can become very large for the heavy elements $(10 \mathrm{eV}$ splitting between the $p_{1 / 2}$ and $p_{3 / 2}$ orbitals in Og with $\left.Z=118\right)^{77}$ (see Figs.4a,b). These large relativistic effects have been overlooked for a long time, but are perhaps not unexpected as 
these usually scale $\sim Z^{2}$ for the valence shells of analogous elements.

A detailed account on relativistic shell-structure effects in the $d$ and $f$ blocks of the PTE has been given by Schwarz and co-workers ${ }^{63}$. Here, we focus on the two late transition metals, $\mathrm{Au}$ and $\mathrm{Hg}$. It is now well established that these elements exhibit unusually large relativistic $6 s$-orbital stabilizations (contractions) and indirect relativistic $5 d$-orbital destabilizations (expansions) compared to their lighter congeners and to their neighbors to the left and right in the PTE, as first shown by Desclaux ${ }^{78}$ and discussed in Ref. 71. A maximum of the stabilization of the $n s$ shell can be observed for the $(n-1) d^{G-1} n s^{1}$ configuration in group 11 and for the $(n-1) d^{G-2} n s^{2}$ configuration in group 12 , where $G$ is the group number (Fig.4c). The maximum in group 11 is well known ${ }^{71}$, but perhaps not well understood ${ }^{79}$. It originates from the successive filling of the underlying $(n-1) d$ shell $^{63}$.

For the following discussion we define relativistic effects for a specific atomic property $P$ as $\Delta_{R} P=\left(P_{R}-P_{N R}\right)=\gamma_{P}\left(Z \alpha_{\mathrm{EM}}\right)^{2} P_{R}$, where $\gamma_{P}$ is the relativistic enhancement factor ${ }^{63}$. Although down a group relativistic effects mostly follow the expected $\sim Z^{2}$ behaviour, for group 11 the relativistic enhancement factor is very large and increases for the heavier elements. For example, we calculate $\gamma_{\epsilon}$ values ${ }^{80}$ for the orbital energy $\epsilon_{n s}$ in group 11 to be $0.573(\mathrm{Cu}), 0.616(\mathrm{Ag}), 0.731(\mathrm{Au})$, and $0.795(\mathrm{Rg})$ versus the values for group 12 that are $0.428(\mathrm{Zn}), 0.480(\mathrm{Cd}), 0.616(\mathrm{Hg})$, and $0.706(\mathrm{Cn})$. The large relativistic enhancement factors for $\mathrm{Au}$ and $\mathrm{Hg}$ give rise to well-known anomalies in their chemical and physical behaviour, some of which for $\mathrm{Au}$ are detailed in Box 4. These anomalies are predicted to 
be even more pronounced in superheavy element Rg (roentgenium), the chemistry of which remains largely unexplored despite it being discovered in $1994^{11,81}$. The ground state of $\mathrm{Rg}$ has a $6 d^{9} 7 s^{2}$ as opposed to a $6 d^{10} 7 s^{1}$ configuration owning to the very large relativistic $7 s$ contraction that makes Rg as small as copper in size ${ }^{82-84}$. It is clear from the data in Box 4 that it is almost impossible to predict the physical and chemical behaviour of Rg from the properties of its lighter group members. In this case, we cannot count on the concept of chemical similarity within a group. We note, however, that relativistic effects heavily depend on the $n s$ population of the elements in a molecule or the solid ${ }^{85,86}$.

Turning to the $\mathrm{Hg}$ in group 12, we also observe large relativistic effects that lead to many abnormal physical and chemical properties ${ }^{87}$. Perhaps the most striking property is that $\mathrm{Hg}$ is the only elemental metallic liquid in the PTE (followed by gallium that has a melting temperature of $29.76^{\circ} \mathrm{C}$ ) with a very high density of $13.5 \mathrm{~g} \mathrm{~cm}^{-3}$. Theoretical studies have shown that relativistic effects lower the melting point of $\mathrm{Hg}$ by more than $100 \mathrm{~K}$ (105 $\mathrm{K}$ using the atom-in-molecules method ${ }^{88}$ and $160 \mathrm{~K}$ using $\mathrm{DFT}^{89}$ ). Thus non-relativistic $\mathrm{Hg}$ would be a solid at room temperature, like Cd. The heaviest known group-12 element, $\mathrm{Cn}$, has recently been predicted to be a liquid at room temperature too owing to relativistic effects, with a rather narrow liquid range below the boiling point ${ }^{90}$, thus supporting Pitzer's original hypothesis ${ }^{91}$.

Because of the strong relativistic $7 s$ contraction, $\mathrm{Cn}$ is predicted to be a semi-conductor or even an insulator in contrast to $\mathrm{Hg}^{90,92}$. The unusually high superconducting transition 
temperature of $\mathrm{Hg}$ as compared to that of $\mathrm{Zn}$ and $\mathrm{Cd}$ is also attributed to relativistic effects ${ }^{93}$ — without relativity, Heike Kamerlingh Onnes would not have discovered superconductivity. Note that the specific resistivity of $\mathrm{Hg}$ is unusually high, 95.78 (in $10^{-8} \Omega \mathrm{m}$ ) compared to Zn (5.8) or Cd (7.6).

An interesting property of $\mathrm{Hg}$ and its superheavy group member $\mathrm{Cn}$ is that they can both adopt the oxidation state $+\mathrm{IV}{ }^{83,94}-\mathrm{HgF}_{4}$ has been identified not too long ago by Wang and co-workers ${ }^{95}$. The chemistry of $\mathrm{Cn}$ has recently been explored by using atom-ata-time adsorption on gold surfaces, suggesting that this element is very volatile ${ }^{96,97}$.

[H2] Spin-orbit effects in the $p$-block elements. Fig.4d demonstrates the size of spinorbit splitting in individual $l>0$ shells for Og. It diminishes for shells with higher angular quantum number $l$ (as predicted by the Dirac equation), and is especially large for the lowest and highest principal quantum number $n$, see Fig.4a. Spin-orbit splittings for the group 13, 14, 16 and 17 elements follow a $\sim Z^{2}$ behaviour (Fig.4d) and for period 6 its effects become already large enough to influence chemical bonding. However, during the formation of covalent bonds, spin-orbit effects can be substantially suppressed by the mixing of $p_{1 / 2}$ and $p_{3 / 2}$ orbitals to form $\sigma$ or $\pi$ bonds. For example, the combination of two $p_{1 / 2}$ orbitals at different atomic centres gives $1 / 3$ of $\sigma$ bonding and $2 / 3$ of $\pi^{*}$ antibonding or, switching sign, $2 / 3 \pi$ bonding and $1 / 3$ of $\sigma^{*}$ antibonding. Additionally, combining two $p_{3 / 2}$ orbitals at different centres results in one $\pi$ bond or a combination of $2 / 3 \pi$ bonding and $1 / 3$ of $\sigma^{*}$ antibonding for $m_{j}=3 / 2$ and $1 / 2$, respectively ${ }^{72,91,98}$ This mixing also occurs in external 
fields, for example, spin-orbit effects can be suppressed in strong ligand fields during the formation of ionic bonds. Furthermore, electron density can move from the central atom to the electronegative ligand thus diminishing spin-orbit effects, especially in the case of compounds in high oxidation states.

Spin-orbit effects are also the reason for a very small dissociation energy in $\mathrm{Tl}_{2}(41$ $\left.\mathrm{kJ} \mathrm{mol}^{-1}\right)^{99}$ and consequently a rather small cohesive energy for the bulk $\left(182 \mathrm{~kJ} \mathrm{~mol}^{-1}\right)$ and low melting point $\left(304^{\circ} \mathrm{C}\right)$. This large spin-orbit destabilization is even more evident for the heaviest element dimer in group $13, \mathrm{Nh}_{2}$, in which the compact $7 p_{1 / 2}$ shell and low population of the $7 p_{3 / 2}$ orbital lead to a dissociation energy of only a few $\mathrm{kJ} \mathrm{mol}^{-1}$ despite of the combination of two open-shell atoms ${ }^{100,101}$. Fl $(Z=114)$ has a closed spherical $7 p_{1 / 2}^{2}$ shell well separated from the much higher lying $7 p_{3 / 2}$ shell, Fig.4d. Solid-state calculations indeed show that bulk Fl has a very small cohesive energy of $49 \mathrm{~kJ} \mathrm{~mol}^{-1}$ (down from $291 \mathrm{~kJ}$ $\mathrm{mol}^{-1}$ at the scalar relativistic level where spin-orbit coupling is neglected) compared to $\mathrm{Pb}$ with $195 \mathrm{~kJ} \mathrm{~mol}^{-1} .{ }^{102}$ This is supported by atom-at-a-time adsorption experiments of $\mathrm{Fl}$ on a gold surface that indicate a higher inertness compared to its lighter group members resulting in a high volatility ${ }^{103,104}$. According to these studies, Fl is the least reactive element in the group, but is still metallic in nature, and may be a liquid at room temperature.${ }^{91}$.

It is well known that spin-orbit coupling is important for the correct interpretation of electronic spectra in atoms, molecules and the solid state. The photo-electron spectra of $\operatorname{Bi} X_{3}(X=\mathrm{Cl}, \mathrm{Br}, \mathrm{I})$ may serve as an interesting example ${ }^{105}$. In this case, the bismuth- 
halogen $\sigma$ bonding orbitals suffer from a relativistic rehybridization due to spin-orbit coupling, and the energetic order of peaks is dominated by the central-atom spin-orbit splitting $\epsilon\left(6 p_{1 / 2}\right)<\epsilon\left(6 p_{3 / 2}\right)$. In materials science, the Bi spin-orbit coupling helps to synthesize new high-pressure intermetallics ${ }^{106}$.

As a last example we mention the noble-gas element $\mathrm{Og}(Z=118)$ (Fig. 4a,b), the last known $p$-block element and currently the heaviest element in the PTE. Here, the ${ }^{2} P_{3 / 2} /{ }^{2} P_{1 / 2}$ splitting in $\mathrm{Og}^{+}$is about $10 \mathrm{eV}$ and larger than most bond dissociation energies. In fact, $\mathrm{Og}$ is quite different compared to the lighter rare gas atoms with an electron localization function comparable to a Fermi gas ${ }^{77}$ (Fig.5a). Og is predicted to be a solid under ambient conditions due to relativistic effects ${ }^{107}$, exhibiting some unusual chemical and physical properties ${ }^{107-110}$, such as positive electron affinity of $0.056 \mathrm{eV}^{111}$.

[H2] The inert-pair effect. The lowering of oxidation states in the $6 p$-block elements such as $\mathrm{Pb}$ (II) compared to $\mathrm{Sn}(\mathrm{IV})$ — is linked to the so-called inert-pair effect, introduced by Sidgwick ${ }^{112,113}$. In short, the inert pair effect is the tendency of the two electrons in the outermost atomic s-orbital to remain mostly unshared or localized in compounds of posttransition metals. Here, the increasing nuclear charge down a group leads to the contraction of valence $n s$-shell and, therefore, to a large separation between the $n s / n p$ levels and to reduced mixing (hybridization) between the two, thus altering chemical bonding ${ }^{114}$. Actually the relevant hybridization also includes the orbitals of the ligands. ${ }^{72}$ Thus the higher oxidation state becomes unfavourable as seen, for example, in the group 13 and 14 hydrides $^{115-117}$, 
or in the group 14 chlorides shown in Fig.4e.

A striking example of the inert-pair effect is provided by the lead-acid battery, because a great part of the voltage arises from relativistic effects ${ }^{118}$. The largest relativistic contribution comes from the high-energy compound $\mathrm{Pb}^{\mathrm{IV}} \mathrm{O}_{2}$ in the lead-acid battery reaction $\mathrm{Pb}(s)+\mathrm{PbO}_{2}(s)+2 \mathrm{H}_{2} \mathrm{SO}_{4}(\mathrm{aq}) \rightarrow 2 \mathrm{PbSO}_{4}(s)+2 \mathrm{H}_{2} \mathrm{O}(l)$. This is shown relativistic shifts of energies of formation $E_{f}$ for $\mathrm{Sn}$ and $\mathrm{Pb}$ compounds and for $\mathrm{SO}_{3}$ relative to the nonrelativistic (NR) energies (scalar relativistic and fully relativistic (including spin-orbit) effects defined as $\Delta_{\mathrm{SR}}=E_{f}(\mathrm{SR})-E_{f}(\mathrm{NR})$ and $\left.\Delta_{\mathrm{FR}}=E_{f}(\mathrm{FR})-E_{f}(\mathrm{NR})\right)$. Using Faraday's law, $\Delta G^{0}=-R T \log (K)=-n F E_{\text {cell }}^{0}$, about $1.74 \mathrm{~V}$ of the total experimental cell voltage of 2.107 $\mathrm{V}$ is attributed to relativity.

For a more detailed discussion on the inert-pair effect including an historical account see Ref.114. Further information on periodic trends in the main groups can be found in a recent paper by Frenking and co-workers ${ }^{19}$.

[H2]The lanthanides. Lanthanides $(\mathrm{Ln}=\mathrm{La}-\mathrm{Lu})$ are the elements in which the $4 f$ shell is gradually filled. Typical Ln atom configurations are $4 f^{G-3} 5 d^{1} 6 s^{2}$ or $4 f^{G-2} 6 s^{2}$ ( $G$ is the group number). Unlike the $d$-block elements, the compact $4 f$ electrons are little involved in chemical bonding and act as 'spectators'. The $4 f$ electrons can thus be treated (to a certain extent) as core-like. The formation of chemical bonds between Ln and other elements mainly involves Ln $6 s$ and $5 d .{ }^{119,120}$ This is the main reason for the chemical similarity of the lanthanides - many of us know how hard it is to separate the different lanthanides. Note 
that it is not important if the $5 d$ shell is occupied in the atomic ground state, as long as it is energetically available.

The $4 f$ electrons do however have a substantial influence on the physical properties of the lanthanide compounds because the $4 f$ shell does not completely shield the nucleus. This leads to the so-called lanthanide contraction. With the increase of the nuclear charge, the ionic radius of $\mathrm{Ln}^{3+}$ decreases from La to Lu leading to progressively smaller bond distances in lanthanide compounds. The lanthanide contraction was introduced by Goldschmidt ${ }^{121}$, who referred to both the contractions of radii in the series La-to-Lu, as well as when going from pre- $4 f$ elements to post- $4 f$ elements (for example, Ag versus $\mathrm{Au}$ ). Further, the successive filling of the $4 f$ shell has a significant influence on the $6 s$ shell, for example, the relativistic stabilization of the $6 s$ shell for La is $5.4 \%$ compared to $11.9 \%$. The core-like $4 f$ states play a role in magnetic and optical properties of the lanthanides ${ }^{122}$. Lanthanides are mostly trivalent, except in cases like $\mathrm{Eu}(\mathrm{II})$ or $\mathrm{Yb}(\mathrm{II})$ in which half-filled or filled $4 f$ shells favour divalency. Recently, low-valent compounds have been synthesized by W. J. Evans and colleagues ${ }^{123}$.

[H2] The actinides. We analogously label actinides as An=Ac-Lr. The later actinides after Am are mostly trivalent and chemically similar to their Ln counterparts, creating problems in the chemical treatment of nuclear waste. In the range $\mathrm{Th}-\mathrm{Am}$ a rich hybridization and multiple bonding can occur, notably to $\mathrm{N}$ or $\mathrm{O}$ ligands, with the actinyl group being a prime example $^{124}$. The main contributing An orbitals are $6 d$ and $5 f$, with possible participation of 
the diffuse $7 s$ and $7 p$, and the semicore $6 p$. It should be noted that the $5 f$ shell has a larger radius than the $4 f$, both because it has a radial node and because it experiences an indirect relativistic destabilisation. There has been a long standing debate on the involvement of the $5 f$ electrons in chemical bonding in certain compounds. For example, recent experimental and theoretical studies of Am-Cf consider systems with a half-filled $5 f$ shell, such as Bk(IV) or $\mathrm{Cf}(\mathrm{v})$, and divalent $\mathrm{An}(\mathrm{II})$ compounds, in both non-aqueous and aqueous systems ${ }^{125,126}$. Both the An $6 d$ and $5 f$ orbitals are found to be involved. The debate on the role of $5 f$ electrons focused more on the earlier actinide elements $U$ and $N p$, that do not exhibit a proper actinide contraction ${ }^{127}$. As said, the insertion of the $f$-elements into the periodic table and the resulting partial shielding of the nucleus have a profound influence on the chemistry of the post- $f$ elements. Furthermore, relativistic effects increase the actinide contraction substantially. Similar to the lanthanides, the relativistic stabilization of the $7 \mathrm{~s}$ shell for Ac is $18.4 \%$ compared to the $35.1 \%$ for Lr (Fig.4c).

A more detailed discussion on shell-structure and relativistic effects for the lanthanides and actinides can be found in Refs. 98,128,129. For the discovery history of the elements 93-118, see Chemey and Albrecht-Schmitt ${ }^{130}$.

[H2] Beyond the known elements. How the PTE can be expanded beyond the currently known elements $(Z>118)$ has been discussed recently by Pyykkö ${ }^{10,131}$. The two elements that follow Og are the 8 s-elements below Fr and Ra, which have yet to be discovered. For details on current attempts carried out at GSI (Germany), Dubna (Russia) and RIKEN 
(Japan) laboratories to synthesize and detect superheavy elements beyond nuclear charge 118 the interested reader is referred to Refs $132-136$. So far, we have not discussed the chemistry of the alkali and alkaline-earth metals ${ }^{19}$ because the properties of these elements are expected to become anomalous only for the heaviest two elements with $Z=119$ and $120{ }^{137-140}$ After these two elements one can nominally place the eighteen $5 g$ elements $121-$ $138^{10}$. The atomic number at which the $5 g$ shell begins to be populated is not clear, because electrons may alternatively fill $8 s, 8 p_{1 / 2}, 7 d$ and $6 f$ orbitals. This problem has already been noted by Seaborg in the late $1960 \mathrm{~s}^{141}$. According to one-valence-electron Dirac-Fock calculations, $5 g$ becomes the lowest level from about $Z=125^{10}$. This approximate beginning of some $5 g$ atomic occupation has been known since the 1960ies and used by Seaborg ${ }^{141}$ to discuss the PTE. As we count Th as an actinide even though its $7 s^{2} 6 d^{2}$ atomic ground state has no $5 f$ electrons, let us welcome the elements 121-124 among the $5 g$ elements.

Near the end of the $5 g$ elements, the $8 p_{1 / 2}$ elements $139-140$ follow, then $6 f$ elements 141-155 (note we placed 15 elements here) succeeded by the $7 d$ elements $156-164$, (Fig.1c). The density of electronic states in these elements becomes high. Therefore, in 1971 Fricke, Greiner and Waber suggested an alternative long-row model for their neutralatom Dirac-Slater calculations in which overlaps between different shell occupations are allowed $^{142}$. Fricke and co-workers then placed the $9 s$ elements at $Z=165-166$, the $9 p_{1 / 2}$ elements $167-168$ and finally the $8 p_{3 / 2}$ elements $169-172$. The neutral-atom multi-configuration Dirac-Fock (MCDF) calculations of Indelicato and colleagues support the order arising from the Dirac-Slater calculations but exchanges $8 p_{3 / 2}$ with $9 p_{1 / 2}{ }^{143}$, and also add element 173 . 
The chemistry of these elements still needs to be explored, including the correct assignment for the lowest (ground) state and low-lying excited states and corresponding share of configurations. This requires high-level multi-reference theory, including QED $^{22,143}$. This is basically unexplored territory and a major challenge for atomic structure theory ${ }^{38}$. A first DFT attempt to study the chemistry of the $5 \mathrm{~g}$ elements shows that the hypothetical octahedral hexafluorides, $\mathrm{MF}_{6}$, of the elements with nuclear charge $Z=125-129$ prefer occupied compact $5 g$ (spectator) states similar to the $4 f$ shell in the lanthanides. ${ }^{144}$ As the $5 g$ shell is even more compact than the $\operatorname{Ln} 4 f$ shells $^{10}$, the $5 g$ series would deserve the name of superlanthanides. ${ }^{8}$

[H2] The critical nuclear charge. The PTE in Fig.1c finishes with the last entry at $Z=172^{10,142}$, while MCDF+QED calculations indicate element 173 to be the heaviest one $^{143}$. It is often believed that the PTE (from an electronic point of view) cannot be expanded beyond a critical nuclear charge $Z_{\text {crit }} \approx 170$, a value above which atoms can no longer be described by the stationary Dirac-Coulomb equation ${ }^{145}$. According to the hydrogenic expression for the total energy arising from the Schrödinger equation we have $E_{n}^{\mathrm{NR}}=-Z^{2} /\left(2 n^{2}\right)$ (in atomic units), that is the energy decreases quadratically with nuclear charge (Fig.4f). The corresponding Dirac equation for a point charge nucleus (PCN) affords a different analytical expression of the energy,

$$
E_{n j} / m_{e} c^{2}=\left(1+\frac{\left(Z \alpha_{\mathrm{EM}}\right)^{2}}{\left[n-j-1 / 2+\sqrt{(j+1 / 2)^{2}-\left(Z \alpha_{\mathrm{EM}}\right)^{2}}\right]^{2}}\right)^{-1 / 2}-1
$$


with $j=l \pm 1 / 2$. Fig. 4 f shows that the non-relativistic energy starts to deviate substantially from the relativistic value beginning at $Z \approx 60$ for the $1 s$ orbital and $Z \approx 80$ for the $2 s$ orbital. This deviation originates from the relativistic operators that act in the vicinity of the nuclei and from the $s$-orbitals having higher electron density near the nucleus. However, the hydrogenic PNC-Dirac equation has no longer valid solutions for $Z>Z_{\text {crit }}^{\mathrm{PNC}}=\alpha_{\mathrm{EM}}^{-1}(j+1 / 2)$, that is for $Z>137$ for $s$ states, $Z>274$ for $p_{3 / 2}$ and $d_{3 / 2}$ states, and so on, as the total energy becomes imaginary. In mathematical terms, the Dirac operator is no longer self-adjoint; this happens even earlier for real eigenvalues at $Z=118^{146,147}$. The energy curves, therefore, end abruptly at $E_{\text {crit }}^{1 s}=-m_{e} c^{2}$ and $E_{\text {crit }}^{2 s}=-m_{e} c^{2}(1-1 / \sqrt{2})$. However, the introduction of a finite extension of the nuclear charge (FNC) distribution removes this unphysical behaviour 148,149 (Fig.4f). A comparison between the energy curves produced using the PNC and FNC models reveals that the PNC model becomes inaccurate for $Z>120$ for the $1 s$ state and $Z>130$ for the $2 s$ state. However, disaster strikes again when these states enter (dive into) the negativeenergy continuum, the Dirac sea, at $Z_{\text {crit }}^{\mathrm{FNC}}>170$ for the $1 s$ state and at much larger $Z_{\text {crit }}^{\mathrm{FNC}}$ value for the $2 s$ state (note that the value of $Z_{\mathrm{crit}}^{\mathrm{FNC}}$ depends on the nuclear charge distribution used)(Fig.4f). A remedy to this situation does not come easy and possible solutions have been proposed ${ }^{150}$. One possible explanation to this problem is that PTE does not end at some $Z_{\text {crit }}$. The description of multi-electron systems using the bare Dirac equation without the full QED framework in supercritical Coulomb fields provides an incomplete picture (note that for multi-electron systems the electron screening of the nucleus shifts $Z_{\mathrm{crit}}^{\mathrm{FNC}}$ to higher values, ${ }^{145}$ but the diving is not avoided). In fact, the 1 s state at $Z>Z_{\text {crit }}^{\mathrm{FNC}}$ is embedded in 
the Dirac continuum, the vacuum now becomes charged. ${ }^{150}$ In a (multi-electron) system, the diving of an open shell will cause electron-positron pair creation, which should be observable in collisions of highly charged nuclei resulting in a compound nucleus beyond the critical charge $^{151}$. Besides, in this region of high nuclear charge we expect the nuclear instability to be an 'earlier killer' as we shall see in section 'Stability of superheavy elements' ${ }^{\text {'11. }}$

\section{$4 \quad[\mathrm{H} 1]$ Astrophysical nucleogenesis}

Around 13.8 billion years ago, within approximately a second from the Big Bang, the most abundant elements in our universe were $\mathrm{H}\left({ }_{1}^{1} \mathrm{H}\right.$ and $\left.{ }_{1}^{2} \mathrm{H}\right)$ and $\mathrm{He}\left({ }_{2}^{4} \mathrm{He}\right.$ and $\left.{ }_{2}^{3} \mathrm{He}\right)$, with trace amounts of Li and some heavier elements also being present (Fig.1d). All were formed from free neutrons and protons, radiative capture, neutrino interactions and subsequent nuclear fusion and decay reactions from the product isotopes. Under the influence of their mutual gravitational forces, the clouds of atomic $\mathrm{H}$ and $\mathrm{He}$ gas contracted forming the first stars after 100 million years or so, leading to high temperatures and pressures in these population III stars, stars that were composed entirely of primordial gas. Through nuclear fusion in these young stars, heavier elements (up to iron), including the isotopes essential to life ${ }_{6}^{12} \mathrm{C}$ and ${ }_{8}^{16} \mathrm{O}$ ), were formed, and continue to form in existing stars. For elements lighter than iron, nuclear fusion releases energy when forming the most stable isotopes. Instead, for the heavier elements, the nucleus is so tightly bound that nuclear fusion consumes energy. The fusion process of the star is thus halted when the silicon burning phase has produced an iron core and, as a result, the star collapses under its gravitation force. Above a certain critical 
mass, when the star's core becomes as dense as an atomic nucleus, it rebounds explosively as a so-called supernova, releasing a flux of neutrons, protons and atoms into interstellar space, and finally a neutron star or black hole is formed.

Before we proceed with any further details, we point out that the efficient synthesis of $\mathrm{C}\left(3{ }_{2}^{4} \mathrm{He} \rightarrow{ }_{6}^{12} \mathrm{C}\right), \mathrm{O}\left({ }_{6}^{12} \mathrm{C}+{ }_{2}^{4} \mathrm{He} \rightarrow{ }_{8}^{16} \mathrm{O}\right)$ and other elements in stars requires a rather delicate tuning of two fundamental constants: the fine structure constant $\alpha_{\mathrm{EM}}$ and the strong coupling constant $\alpha_{\mathrm{S}}$, which enter amongst other coupling constants the standard model in physics. Oberhummer and colleagues showed that if $\alpha_{\mathrm{S}}$ varies by $0.5 \%$ and $\alpha_{\mathrm{EM}}$ by $4 \%$, the stellar production of $\mathrm{C}$ or $\mathrm{O}$ will be reduced by a factor $30-1000^{152,153}$. Furthermore, the neutronproton mass difference $\Delta_{n p} m=m_{n}-m_{p}$ is also sensitive to a change in $\alpha_{\mathrm{EM}}$ or $\alpha_{\mathrm{S}}$. Borsanyi and co-workers showed that a value of $\Delta_{n p} m<0.45 \mathrm{MeV} c^{-2}$ would cause $\mathrm{H}$ (proton) to undergo inverse $\beta$-decay (electron capture), resulting in predominant presence of neutrons in our universe ${ }^{154}$. A value of $\Delta_{n p} m \sim 0.5 \mathrm{MeV} c^{-2}$ would have resulted in the Big Bang nucleosynthesis producing much more ${ }_{2}^{4} \mathrm{He}$ and far less $\mathrm{H}$ than it did in our universe. A considerably large value of $\Delta m_{n p}=1.3 \mathrm{MeV} c^{-2}$ would have resulted in a faster $\beta$-decay for neutrons leading to far fewer neutrons at the end of the Big Bang nucleosynthesis, making the burning of $\mathrm{H}$ in stars and the synthesis of the heavy elements difficult ${ }^{154}$. Thus, the existence of the elements in our universe is dictated by the fine-tuning of these fundamental constants, which we can measure very precisely but are yet to understand ${ }^{155}$. The variation of fundamental constants in space-time might be linked to the existence of dark matter, and this is currently an active field of research both in physics and chemistry ${ }^{156,157}$. 
Returning to the astrophysical nucleosynthesis, a small percentage of the elements heavier than iron is produced by rapid proton capture (the $r p$-process in binary star systems involving a neutron star where high temperatures above $10^{9} \mathrm{~K}$ can be achieved to overcome the Coulomb barrier in nuclear reactions. This $r p$-process produces proton-rich nuclei that can be identified on the right of the proton stability (drip) line shown in the $(N, Z)$ (Segré) chart $^{158-163}$ in Fig.6a, reaching elements up to (perhaps) tellurium ${ }^{164-166}$ (note that at the proton (neutron) drip line the nuclei are at the very edge of particle stability such that they emit protons (neutrons) directly; drip-line isotopes provide very useful information for nuclear structure theory). However, most of the heavy elements in the PTE are produced by neutron-capture processes, as first suggested by Gamow ${ }^{167}$ in 1946 and by Alpher and Herman ${ }^{168}$ in 1950 . Neutron-capture occurs on a time-scale of roughly $1 \mathrm{~s}$, three orders of magnitude smaller than the neutron half-life $\left(t_{1 / 2}=883 \pm 7 \mathrm{~s}\right)$ that dictates its decay into a proton, an electron and an antineutrino ${ }^{169-171}$. In the nucleosynthesis process, neutrons are successively absorbed by a nucleus creating isotopes on the left of the neutron drip lines shown in the Segré chart in Fig.6a. At a certain mass number, the neutron-rich isotope will then $\beta$ decay to a more stable nucleus with the nuclear charge increased from $Z \rightarrow Z+1$. This process repeats up to a nuclear charge $Z$ that depends on the initial astrophysical conditions and the stability of the daughter nucleus at the end of the chain. Fig.6a shows such a possible path (red line) for the rapid neutron capture process.

There are many possible astrophysical sites of neutron-rich matter (see discussion below) and many different processes and conditions ${ }^{172,173}$. However, for the synthesis of the 
heavy elements, we can identify four important types of neutron-capture processes based on different astrophysical conditions: the rapid-neutron capture ( $r$-process) with number of neutrons $N_{n}>10^{20} \mathrm{~cm}^{-3}$ (for comparison this approximately equals the number of ideal gas molecules under normal conditions), ${ }^{174}$ the $n$-process when $N_{n} \approx 10^{18} \mathrm{~cm}^{-3},{ }^{175}$ the intermediate neutron capture $\left(i\right.$-process) when $N_{n} \approx 10^{15} \mathrm{~cm}^{-3},{ }^{176,177}$ and the slow neutron capture (the $s$-process) with $N_{n}=10^{6}-10^{10} \mathrm{~cm}^{-3} 178$. These conditions define the different reaction paths and average time necessary before a neutron-rich isotope $\beta$-decays to a more stable nucleus with increased nuclear charge. Whereas s-capture produces nuclei near the valley of $\beta$ stability, for the $r$-process the reaction path is shifted into the neutron-rich region of the nuclide chart because neutron capture-times in this case are much shorter than the average $\beta$ decay. Because of the loss of stability for elements heavier than Bi (the $s$-process terminates at ${ }_{84}^{210} \mathrm{Po}$ ), isotopes like $\mathrm{U}$ and $\mathrm{Th}$ can only be generated through a $r$-capture process. In this cases, the $s$-capture would be too slow and the intermediate nuclei decay before enough neutrons are captured to reach higher $Z$ values $^{179,180}$ (Box 7).

The two main neutron capture processes, $r$ and $s$, produce different isotope distributions, therefore it is possible to identify the abundancy distribution for different heavy elements by their production type. For example, by comparing the isotopic abundance distribution with predictions from analytical models and nuclear reaction networks coupled to stellar evolution codes, it is predicted that about $51 \%$ of the heavy elements in the solar system originated through $s$-process nucleosynthesis, with the remaining $49 \%$ produced by other nucleosynthesis mechanisms, primarily the $r$-process ${ }^{181}$. An example of a comparison 
between experimental and predicted abundance distributions of elements that are produced by the $r$-capture and $s$-capture in the metal-poor star HD108317 is shown in Fig.7a, where we see that the $s$-capture process terminates at ${ }_{83} \mathrm{Bi}$ (blue line in Box 5 ).

Abundancy distributions using theoretical stellar evolutionary models were first predicted in the late 1950-early 1960s by Burbidge, Clayton, Seeger and colleagues ${ }^{164,182-184}$. It was shown how, from the sequence of abundance distributions generated for specific numbers of neutrons per initial seed nucleus, one can estimate the superpositions of neutron exposures required to reproduce the experimentally observed abundance distributions of the isotopes produced through the $s$-process. An improved fit of simulation models to experimental data was later obtained by including more accurate evolution models and by integrating the full reaction network in the simulation ${ }^{185-187}$. Although the relative abundance distributions of the heavy elements in our universe obtained from numerical simulations nicely align with experimental results, it is very complicated to identify the astrophysical sites where the $s$-processes and $r$-processes take place. One relies on nucleosynthesis models that are dependent on nuclear physics inputs and the astrophysical conditions to get an idea on the origin of such processes. Distinguishing different astrophysical scenarios is challenging because the exact properties of the nuclear states of the isotopes involved in the capture process are difficult to obtain and the exact conditions of the ejecta are unknown. The easiest approach is to study events that happened shortly after the Big Bang because nearly all older stars contain traces of products of the $r$-process. Another obstacle is the difficulty to obtain information about exact stellar conditions. When nuclear excited states are thermally populated (which 
happens in stars, but not in the laboratory) neutron capture can proceed not only for nuclei in their ground states, but also from nuclear excited states and the reaction rates under stellar conditions are thus different from those measured in laboratory. For these reasons, sites and mechanisms of neutron capture production are still lacking substantial abundance observations to constrain proposed theoretical models.

First suggested by Ulrich ${ }^{188}$, multiple neutron captures could take place in the Herich layer of asymptotic giant branch (AGB) stars. During the late phase of the AGB, the thermal instability of the He burning-shell leads to burning flashes of high amplitudes that establish convection zones that modify the distribution of He throughout the affected layers. As a result, $\mathrm{H}$ is reintroduced into hot layers providing the flux of neutrons for the neutron capture process ${ }^{183,189,190}$. Current models suggest the existence of two components to the $s$-process. The main s-component involves stars during their AGB phase (when they are in the mass range between 1 and 3 solar masses) that are responsible for the production of the isotopes in the mass region between $\mathrm{Zr}$ and $\mathrm{Bi}$ (mass number $A>90$ ) ${ }^{185,191}$. The weak s-component occurs in massive AGB stars larger than 8 solar masses that are thought to be responsible for the production of elements between Fe and $\operatorname{Zr}(60<A<90)^{192,193}$. The existence of a third component was proposed after discovering that the main and weak $s$ component do not fully reproduce the $s$ abundances between $\mathrm{Sr}$ and $\mathrm{Ba}{ }^{194}$.

The $i$-process is thought to occur in carbon enhanced metal poor (CEMP) stars and the $n$-process in supernova shock front traversing the He burning shell ${ }^{175-177}$. Concerning the $r$ - 
capture process, there are a number of possible astrophysical environments. It was originally thought that $r$-capture occurs in supernovae ${ }^{195}$. Recent studies suggest neutron sources from kilonovae (merging neutron stars), because the $r$-process requires extreme conditions, although, theoretical models do not exclude supernovae as sites of heavy $r$-processing ${ }^{172,196-199}$. The recently observed GW170817 neutron-star merger produced radiation across the electromagnetic spectrum consistent with the ejection of some hundredths of a solar mass of lanthanide-rich material ${ }^{200,201}$. This confirmed that heavy elements can be forged during such events, suggesting neutron star mergers as primary site for the $r$-process and possible production sites for the lighter actinides ${ }^{202}$ and strontium ${ }^{203}$. The likely different astrophysical origins of the elements ${ }^{164,204,205}$ are illustrated in the PTE in Fig.1d. More experimental and theoretical nuclear structure data will be required in the near future to fully understand the various neutron capture processes and the synthesis of the heavy elements.

Elements heavier than the $\mathrm{U}$ originating from neutron-capture processes have not been observed in larger amounts ${ }^{206,207}$ despite a few searches and claims ${ }^{208,209}$. The main reason is that the fission barrier of the heavy nuclei produced during the $r$-capture process is lowered and, as a result, the heavy nuclei decay before neutron capture takes place. However, it is suggested that the production of heavy nuclei is enhanced if the $r$-process path proceeds closer to the neutron drip line. Petermann and co-workers performed fully dynamical network $r$-process calculations assuming an environment with neutron/seed ratio large enough to produce superheavy nuclei with $Z \geq 104$ and $A \approx 300$ during the first seconds of the $r$ process (Fig.7b) ${ }^{210}$. However, the yields strongly depend on predicted nuclear data and 
astrophysical scenario, and the produced superheavy elements decay on time scales of seconds or minutes ${ }^{211}$. The lifetimes of superheavy nuclei is further discussed in detail in the next section.

\section{$5 \quad[\mathrm{H} 1]$ Stability of superheavy elements.}

The heaviest naturally occurring elements of the PTE on our planet are U, and trace amounts of ${ }^{244} \mathrm{Pu}$ found in the deep sea floor ${ }^{212}$. In fact, up to 1943 only the elements up to $\mathrm{Pu}$, which was produced by a deuteron bombardment of $238-\mathrm{U}$ by Seaborg and co-workers, were known (Fig.1b). At that time, names like 'ultimium' or 'extremium were considered for $\mathrm{Pu}$ because of the erroneous belief that this element might have been the heaviest possible in the PTE $213-215$.

In order to perform chemical experiments, a nucleus containing a given number of protons and neutrons needs to be sufficiently stable with respect to possible radioactive decay channels (from the confinement radius of an electron in an atom, $\sim 10^{-10} \mathrm{~m}$, or a proton inside a nucleus, $\sim 10^{-15} \mathrm{~m}$, and the corresponding masses one can estimate the characteristic time scales for electronic and nuclear reactions using Heisenberg's formula, $\Delta E \Delta t>\hbar$, that are of the order of $\sim 1 \mathrm{fs}$ and $1 \mathrm{zs}\left(\sim 10^{-21} \mathrm{~s}\right)$, respectively $)$. Current experimental approaches require a nuclear stability in the second range, which limits the study of the heaviest elements in the PTE although this might be improved in near future. In fact, at high nuclear charge the PTE is limited not by the electronic but by the nuclear 
stability. Using a simple liquid drop model, Bohr and Wheeler established an upper limit of $Z^{2} / A=47.8(A-Z=N)$ beyond which nuclei become unstable against the elongation of nuclear shape, resulting in spontaneous fission ${ }^{216}$. Using empirical $(Z, A)$ relationships ${ }^{217}$, this sets the upper limit of observable elements to $Z \sim 120-130$, after which the Coulomb repulsion between the protons inside a nucleus becomes too strong ${ }^{218}$. However, while these phenomenological theories based on the liquid-drop model offer great qualitative insight into nuclear properties, a proper treatment of nuclear-shell-structure effects is required to discuss the stability and possible decay channels for certain isotopes, for which the binding energy of the nucleus becomes an important property.

The total mass $M$ of an atom in the PTE can be decomposed into $M\left(Z, N, N_{e}\right)=$ $Z m_{p}+N m_{n}+N_{e} m_{e}-B_{Z, N} / c^{2}-B_{e} / c^{2}$, where $B_{Z, N}$ is the nuclear binding energy for a specific isotope, and $B_{e}$ is the total electronic binding energy $\left(B_{e}=-E_{\mathrm{T}}\right.$ for the total electronic energy) for $N_{e}$ electrons $\left(N_{e}=Z\right.$ for a neutral atom). The mass of a certain isotope can nowadays be determined to $\mathrm{keV}$ accuracy using a trap mass spectrometer or time-of-flight mass spectrometry ${ }^{219-222}$. The proton, neutron and electron masses are all accurately known to eV accuracy. The total electronic energy may be decomposed into $E_{\mathrm{T}}=E_{\mathrm{NRHF}}+\Delta E_{\mathrm{RHF}}+\Delta E_{\mathrm{QED}}+\Delta E_{\mathrm{cor}}$, including the non-relativistic Hartree-Fock total energy (NRHF) ${ }^{223}$, the relativistic energy correction at the Hartree-Fock level including the finite extension of the nucleus $(\mathrm{RHF})^{78}$, QED contributions ${ }^{224,225}$, and electron correlation effects to the many-electron QED Hamiltonian ${ }^{226}$. All these terms can be evaluated to $\mathrm{eV}$ (some of these to meV) accuracy, with the most problematic part being the electron 
correlation contribution that amounts to $\sim 0.4-0.5 \mathrm{eV}$ per electron. This term can, however, be evaluated to an accuracy of $0.05 \mathrm{eV}$ per electron. Hence, with future improvements in mass measurements, the remaining nuclear binding energy can be obtained to at least to $1 \mathrm{keV}$ accuracy. This level of accuracy is important for benchmarking nuclear structure calculations. For example, using nuclear DFT currently has an accuracy of only about 600 $\mathrm{keV}$. Note that the electron binding energy is not so small for the heavier elements compared to the nuclear part, because it increases with $\sim Z^{2}$. For example, for ${ }_{118}^{294} \mathrm{Og}$ the relativistic binding energy is $B_{e}=1.487 \mathrm{MeV}$ compared to the non-relativistic value of $1.260 \mathrm{MeV}^{11,77}$.

A map of nuclear binding energies is shown in Fig.6b, in which we can observe the deviation of the nuclear binding energy from the $Z=N$ line. In other words, if $Z$ increases, more neutrons are required to stabilize the nucleus against Coulomb repulsion. This of course explains the problem in the synthesis of superheavy elements with sufficiently long lifetimes from the beams and target isotopes available. Note that $B_{Z, N}$ varies between $\left[0, B_{\max }\right]$, with the maximum binding energy per nucleon being that for ${ }^{56} \mathrm{Fe}$ with $B_{\max }=8792.23 \pm 0.03 \mathrm{keV}$. There is no such upper limit in electronic structure theory (at least for the non-relativistic case). Here, we point out another fundamental difference between electronic and nuclear structure theory. The electronic binding energy for a $N_{e}$-electron system in the mean-field model follows approximately $B_{e}=\sum_{i=1}^{N_{e}} Z_{\text {eff }}^{2}\left(n_{i}, l_{i}, j_{i}\right) / n_{i}^{2}$ (see discussion above). This description separates energetically the core from the valence shells such that the chemistry of the elements in the PTE is dominated almost exclusively by the valence electrons (except for materials under high pressure ${ }^{227,228}$ ). This is the basis of the 
so-called effective core potential approximation well known to quantum chemists $^{229}$. In nuclear structure theory there is no central field, and we have a 'quantum liquid' of protons and neutrons. This perhaps is best demonstrated by the semi-empirical mass formula for nucleons $^{230}, B_{Z, N}=a_{V} A-a_{S} A^{2 / 3}-a_{C} Z(Z-1) A^{-1 / 3}-a_{A} A^{-1}(A-2 Z)^{2}+\delta(A, Z)$, in which the dominant first (volume) term to the nucleon binding energy is linear in the number of nucleons $A$ (the second term is the surface term, the third the Coulomb term, the forth the asymmetry term and the last one the empirical correction term).

It is beyond the scope of this Review to give an accurate account on current nuclear structure theory (the reader is referred to a recent Review article by Nazarewicz ${ }^{231}$ ). Instead, we point out some of the major differences between nuclear structure theory and electronic structure theory, which is more familiar to chemists, and the challenges that lie ahead in current nuclear structure theory, especially for the treatment of superheavy elements. While the liquid-drop is the earliest (collective) nuclear model introduced originally in the $1930 \mathrm{~s}^{216,232,233}$, it was soon clear that, analogously to electronic structure theory, a nuclear shell model at the microscopic level was needed. In contrast to electronic structure theory, in which the Hamiltonian is virtually known (for example, from the Schrödinger or Dirac-Coulomb equation with the additional corrections arising from QED and hyperfine interactions, containing one-electron and two-electron operators only), the interactions between the nucleons are far more difficult to describe and are based on effective Hamiltonians or functionals. There is no a priori knowledge of the many-body forces between the nucleons and nuclear structure theory becomes an effective field theory. A most rigorous treatment 
at the quark level (lattice quantum chromodynamics) described by the standard model of physics is far too elaborate ${ }^{234,235}$ for a complex many-nucleon system. Hence, one applies different levels of approximations as outlined in Box 6 .

The effective nuclear model Hamiltonian in wave-function-based theories contain parametrized many-body forces, perhaps in some analogy to the treatment of long-range dispersive interactions between atoms or molecules for which, for example, a many-body decomposition of the total energy leads to very accurate results for properties of bulk rare gases $109,236,237$. Such model Hamiltonians can be treated at high-level of theory through configuration interaction or coupled-cluster theory ${ }^{238-241}$ to account for nucleon correlation, but still depend on the accurate description of the many-body forces. As nuclear matter is a strongly correlated quantum liquid, such calculations are expensive and currently applicable only to light and medium sized nuclei ${ }^{242,243}$. This strong correlation also implies that the Hartree-Fock approximation is inadequate for a proper description of nuclear matter. For example, for ${ }_{2}^{4} \mathrm{He}$ coupled cluster calculations for three-body Hamiltonians gave a binding energy of 28.24 $\mathrm{MeV}^{243}$ (extrapolated to the complete basis set limit) compared to $28.2957 \mathrm{MeV}$ (7.0739 $\mathrm{MeV}$ per nucleon, see Fig.6b) obtained from accurate mass measurements ${ }^{244}$ and the mass formula shown above. The current accuracy in most elaborate nuclear structure calculations is of the order of $100 \mathrm{keV}$. Equation-of-motion-coupled-cluster (EOM-CC) theory, originally developed for quantum chemistry ${ }^{245}$, can be used to calculate nuclear excited states. For example, ${ }_{8}^{17} \mathrm{O}$ is a stable isotope with a $(5 / 2+)$ ground state and a low-lying excited $(3 / 2+)$ state at $0.870 \mathrm{MeV}^{246}$ versus $1.219 \mathrm{MeV}$ obtained using EOM-CCSD (which includes single 
and double excitations ${ }^{247}$.

Very similar to the situation in quantum molecular dynamics, for nuclear dynamic calculations it remains a challenge to accurately predict nuclear reactions and radioactive decay channels and corresponding lifetimes such as nuclear fission, $\alpha$-decay and $\beta$-decay (we note that while the free neutron decays with a half-life of 10.3 minutes, ${ }_{2}^{4} \mathrm{He}$ is $\beta$ stable and no such decay is observed because of energy conservation). For example, a comparison between calculated and spontaneous-fission half-lives using the (heavily parametrized) macroscopicmicroscopic (mic-mac) model ${ }^{248}$ including shell corrections for several superheavy elements gave deviations between theory and experiment within three orders of magnitude with errors in binding energies in the $\mathrm{MeV}$ range ${ }^{249}$. Furthermore, tunneling of particles through a potential barrier has an exponential dependence on the barrier height and width, which is well known in chemical dynamics (although, because of the collective dynamic behaviour of nucleons, the notion of a barrier becomes questionable) ${ }^{250}$. Nevertheless, it is difficult to accurately estimate decay properties, including life times. Fortunately, there has been substantial development in nuclear structure theory over the past 20 years that resulted in the improvement of both efficiency and accuracy for the computing of nuclear properties (Box 6). This is especially the case for the nuclear self-consistent mean-field (SCMF) method, which has its roots in $\mathrm{DFT}^{251}$. In this approach, pairing correlation is taken into account using a pairing field obtained from Hartree-Fock-Bogoliubov calculations. For the nucleon potential there are many approximations (such as, Skyrme and Gogny) and parametrizations on nuclear data ${ }^{251}$, very similar to quantum chemistry and the various density functionals. 
From such nuclear structure calculations, one obtains proton and neutron densities $\left(\rho_{p}\right.$ and $\rho_{n}$, respectively), the corresponding nuclear deformations and shell energies. Spin-orbit interactions also need to be taken into account. Extensions beyond mean-field are again similar to those developed in quantum chemistry, like the random phase approximation or multi-reference DFT. A complete overview on this subject has been given by Bender, Heenen and Reinhard ${ }^{251}$, therefore, we focus for the remainder on the mean-field results for the heaviest elements in the PTE.

For nuclear fusion or fission processes, the nuclear mass deformation (mainly quadrupolar and described by the parameter $\left.\beta_{2}\right)^{252}$ is an important parameter that describes the elongation of nuclear shape and often serves as the main reaction coordinates. In fact, in nuclear physics one obtains reaction profiles very similar to chemical kinetics, except over different time-scales $(\sim 1 \mathrm{zs})$ and energy scales $(\mathrm{MeV})$. Fig.6c shows a total energy surface from nuclear structure calculations for the nuclear decay of superheavy element 120 - yet to be synthesized. The picture clearly shows why there was such a long controversy in the past about the 'island of stability', ${ }^{253}$ that is, the compound nucleus lies very high on the energy surface in a rather shallow minimum with high probability for nuclear fission. According to modern theories, lifetimes of superheavy nuclei are fairly short because of fission and $\alpha$-decay; hence, the notion of 'stable superheavy nuclei' is questionable ${ }^{11,12}$. From Fig.6c it is also clear that fusion cross sections are decreased substantially in the superheavy element region as many nuclear collisions will be unsuccessful and end in quasi-fission (QF) processes (QF is characterized by the formation of a composite system that does not reach the fully 
equilibrated compound nucleus) or in products from deep inelastic scattering (DIP). We note that the existence of transactinide nuclei completely relies on shell structure stabilization ${ }^{254}$. Furthermore, above Fm there are not enough neutrons inside the nucleus to reach the most stable predicted isotope, and there are currently no experiments at hand to add additional neutrons after the compound nucleus has formed (unlike in an astrophysical scenario). Hence, the superheavy elements are more on the proton-rich side of the nuclear landscape and they $\alpha$-decay. Furthermore, many of the isotopes are not spherical, but deformed (Fig.6d) ${ }^{255}$. Strong prolate deformations add to nuclear fission instabilities. For example, nuclear localization functions (NLF, similar to electron localization functions ELF) for nuclear fission (cluster decay) of ${ }_{118}^{294} \mathrm{Og}$ are shown in Fig. $5 \mathrm{~b}^{256}$. The NLFs show the overlapping proton and neutron densities in a scission region, where the projectiles just touch, very similar to the overlap of electron densities in chemical reactions. Note that about 100 Og isotopes lie in between the proton and neutron drip lines, a territory that remains to be explored ${ }^{11}$. Another interesting feature in superheavy elements is that, because of Coulomb frustration, the protons are pushed out from the centre of the nucleus (semi-bubble structures), and the high proton and neutron densities of states leave the shells barely visible in nucleon localization functions, in a very similar manner to the electronic case shown in Fig.5a ${ }^{12,77}$. We note that high nuclear stability is obtained around proton and neutron shell closures (so-called magic numbers $2,8,20,28,50$, and 82 for closed proton or neutron shells very similar to magic numbers in electronic shell closures in atoms or clusters), where the nucleus becomes spherical. At higher magic numbers one gets shell gaps at 126 and 184 for neutrons, and 
114124 and 126 for protons. ${ }^{257,258}$ It is worth noting that the notion of magic numbers in superheavy elements is being questioned because of high-level density of single-particle states and Coulomb frustration effects ${ }^{11,12}$.

Nuclear structure calculations are very important for the simulation of the astrophysical synthesis of the elements in the PTE and to predict the right beam and target conditions for the synthesis of new superheavy elements. In so-called cold fusion reactions, the compound nucleus has a relatively low excitation energy such that only few neutrons are emitted and the fission process can be minimized. Targets used are ${ }_{82}^{208} \mathrm{~Pb}$ and ${ }_{83}^{209} \mathrm{Bi}$, and ${ }^{278} \mathrm{Nh}$ (halflife $t_{1 / 2}=2.0_{-0.7}^{+2.7} \mathrm{~ms}$ ) was the heaviest element synthesized at RIKEN laboratory in Japan through the ${ }^{209} \mathrm{Bi}\left({ }^{70} \mathrm{Zn}, n\right)^{278} \mathrm{Nh}$ cold-fusion reaction ${ }^{134,259,260}$. In hot-fusion experiments the projectile nucleus is fixed to ${ }_{20}^{48} \mathrm{Ca}$ and one requires rather heavy actinide targets with nuclei that are well deformed. Such combinations can lead to enhanced cross sections and enabled the synthesis of the elements beyond $\mathrm{Nh}$ up to $\mathrm{Og}$ ( such as, ${ }^{249} \mathrm{Cf}\left({ }^{48} \mathrm{Ca}, 3 n\right){ }^{294} \mathrm{Og}, t_{1 / 2}=$ $0.69_{-0.22}^{+0.64} \mathrm{~ms}$ ) by the Dubna group in Russia ${ }^{261}$. In the hot-fusion experiments, one ends up with a hot nucleus evaporating several neutrons, which is the reason that the hot fusion reaction has been initially overlooked. Two examples of cold and hot fusion reactions are shown in Fig.6e together with a colour coded Segré map of nuclear decay lifetimes that shows enhanced stability at magic numbers $Z=114$ and $N=184$. The given lifetimes and the location of the maximum stability at $Z=114$ has to be taken with care, though, as different nuclear models can predict lifetimes that differ by orders of magnitude, and it is currently not clear if the stability minimum lies at $Z=120,122$ or $126^{11}$. 
There are many Reviews on the experimental synthesis of superheavy elements ${ }^{11,130,262,263}$. We summarize the history of discovery for the superheavy elements in Fig. $6 \mathrm{f}^{130}$, for a historical account and the many controversies surrounding the discovery of the superheavy elements see Ref. 264. The progress towards the synthesis of element with nuclear charge 119 is currently underway at RIKEN laboratory in Japan and both the new Dubna and Darmstadt (GSI) facilities will soon join the efforts in the synthesis of the elements beyond Og. We can expect new elements, perhaps up to nuclear charge 126, in the next decade or so. For this reason, one has to move to heavier beams $\left({ }^{50} \mathrm{Ti},{ }^{51} \mathrm{~V},{ }^{54} \mathrm{Cr}\right)$, because ${ }_{98} \mathrm{Cf}$ is the heaviest target realistically available. It is clear that superheavy elements inhabit the remote corner of the nuclear landscape, but it is not yet clear what happens at much higher nuclear charge. Reliable predictions of lifetimes for different decay channels beyond $Z=118$ are currently not available and it is not known if exotic topologies of nuclear density can produce long-lived isotopes. Also the limits of nuclear mass and charge are currently not known and we expect that nuclear stability will be the limiting factor well below the electronic critical nuclear charge $Z_{\mathrm{crit}}^{\mathrm{FNC}} \cdot{ }^{11,12}$ The binding energies in Fig.6b suggest that the PTE is not infinite, nevertheless, this unknown territory needs to be explored both in terms of nuclear structure theory and quantum chemistry.

\section{$6 \quad[\mathrm{H} 1]$ Conclusions}

The PTE has its solid foundation in quantum theory and is firmly based on both the Pauli and Aufbau principles giving rise to chemical similarities within a specific group. Similar- 
ities between elements in the PTE are dependent on the accessibility of valence electrons for overlap and charge-transfer, as well as on electron-correlation and relativistic effects. Subtle shell structure effects cause irregularities and anomalies in the chemical and physical behaviour of the elements within a specific group. Fuzzy concepts like chemical similarity ${ }^{265}$ often lead to unnecessary disputes concerning the PTE. The ambiguity of clearly assigning a specific element into the right place of the PTE, as for the lanthanides and actinides, shows the limitation of such concepts or approximations ${ }^{266-269}$, but in our opinion should not be over-interpreted as it is sometimes done. We should be reminded that electron configurations arising from the Aufbau principle originate from a mean-field (one-particle) approximation, which can fail for strongly correlated (highly multi-configurational) systems, especially when the density of states becomes large (remember the different electron configurations and dense spectra within the group 10 atoms), as is the case for the transactinides and into the superheavy element region of the PTE. To cite Werner Kutzelnigg: "... one would like to learn more is whether the periodic system has a chance to survive in the realm of superheavy elements"270. Thus, the PTE of elements as suggested recently by one of the authors (Fig.1c) should be seen as a first good approximation, and future theoretical investigations will offer us more insight on the electronic structure of the elements up to the critical nuclear charge $Z_{\text {crit }}$. Beyond $Z_{\text {crit }}$, we need to take into account charged vacuum, which will make a multielectron treatment, including electron correlation, a challenge for future theoretical studies. But this is not the end of the PTE.

Relativistic effects lead to interesting anomalies within the PTE even for atoms with 
the same electron configuration. These effects are enhanced for the elements which exhibit subtle shell structure effects, such as the group 11 and 12 elements $\mathrm{Au}$ and $\mathrm{Hg}$ and their heavier analogs. However, we would not place these elements anywhere else in the PTE because of their dissimilarities to their lighter congeners. Furthermore, spin-orbit coupling in the $p$-block of the PTE has significant influence on their chemical and physical properties. As a curiosity, the element Og can be seen as almost Fermi-like electron gas and is predicted to be a solid at room temperature. Relativistic effects in the region beyond the known elements remain mostly unexplored and we can expect the unexpected here $e^{81,271}$.

Developments in nuclear structure theory and experimental measurements over the past few decades have provided us with very useful data for the prediction of astrophysical synthesis processes (see for example most recent work on the fate of intermediate-mass stars by Kirsebom and colleagues ${ }^{272}$ ) and for many other applications in nuclear physics such as beam-target design in the synthesis of superheavy elements. Superheavy elements have possibly been synthesized in $r$-processes originating from neutron star mergers or supernovae, but have long since decayed and are unlikely to be found on our planet. Over the past 20 years we saw the addition of nine new elements into the PTE, and experiments to obtain elements beyond nuclear charge $Z=118$ are currently underway. The PTE is finite because of nuclear stability and it will be an exciting time to explore the remote corner of the PTE.

The PTE is intrinsically linked to fundamental physics. From a fundamental physics point of view, all four fundamental forces are important for the distribution of the elements in 
our universe and on our planet Earth. Without the electromagnetic force there would be no atoms and without the strong force no nuclei, including the proton and neutron, nor without the weak force any production of the heavy elements through $\beta$-decay. Finally, without the gravitational force no stars would form, and no important elements like carbon and oxygen formed nor accumulated on earth or other planets. Moreover the efficient astrophysical nucleosynthesis process depends on the fundamental constants with a rather narrow range of permitted values to sustain the existence of the elements in the PTE.

We finish with two quotes, the first by Primo Levi (The Periodic Table): "Conquering matter is to understand it, and understanding matter is necessary to understanding the universe and ourselves: and that therefore Mendeleev's Periodic Table, which just during those weeks we were learning to unravel, was poetry." The second by John Emsley (Nature's building blocks: an A-Z guide to the elements): "As long as chemistry is studied, there will be a periodic table. And even if someday we communicate with another part of the universe, we can be sure that one thing both cultures will have in common is an ordered system of the elements that will be instantly recognizable by both intelligent life forms."

Acknowledgements This paper is dedicated to the memory of our friend and colleague Prof. Dr. Werner Kutzelnigg, who recently passed away. We acknowledge financial support by the Alexandervon-Humboldt Foundation (Bonn) and the Marsden Fund (17-MAU-021) of the Royal Society of New Zealand (Wellington). This work is part of the"Molecules in Extreme Environments" project funded by the Centre for Advanced Study at the Norwegian Academy of Science and Letters, 
Oslo, Norway. We thank W. Nazarewicz and Brad Sherrill (Michigan State), Michael Wiescher (Notre Dame), W. H. Eugen Schwarz (Siegen), Yuri Oganessian (Dubna), Gisela Boeck (Rostock), Robert Eichler (Bern), Lukas Pašteka (Bratislava) and L. v. Szentpaly (Stuttgart) for interesting and stimulating discussions. PP acknowledges a travel scholarship from the Magnus Ehrnrooth Foundation.

Competing Interests The authors declare that they have no competing financial interests.

Correspondence Correspondence and requests for materials should be addressed to P. Schwerdtfeger (email: p.a.schwerdtfeger@massey.ac.nz), Pekka Pyykkö (email: pekka.pyykko@helsinki.fi), or Odile Smits (email: smits.odile.rosette@gmail.com).

1. Mendelejew, D. Über die Beziehungen der Eigenschaften zu den Atomgewichten der Elemente. Zeit. Chem. 12, 173 (1869).

2. Van Spronsen, J. W. The periodic system of chemical elements: A history of the first hundred years (Elsevier, Amsterdam, 1969).

3. Kaji, M. DI Mendeleev's concept of chemical elements and the principles of chemistry. Bulletin for the History of Chemistry 27, 4-16 (2002).

4. Scerri, E. R. The periodic table: its story and its significance (OUP USA, 2007).

5. Gordin, M. D. A well-ordered thing: Dmitrii Mendeleev and the shadow of the periodic table (Princeton University Press, Princeton, 2018). 
6. Pushcharovsky, D. Dmitry I. Mendeleev and his time. Substantia 3, 119-129 (2019). URL https://riviste.fupress.net/index.php/subs/article/view/173.

7. Shaik, S., Cremades, E. \& Alvarez, S. The periodic table - a universal icon: Its birth 150 years ago, and its popularization through literature art and music. Angew. Chem. Int. Ed. 58, 13194-13206 (2019). URL https://onlinelibrary.wiley.com/doi/abs/10.1002/anie.201904584. https://onlinelibrary.wiley.com/doi/pdf/10.1002/anie.201904584.

8. Pyykkö, P. An essay on periodic tables. Pure Appl. Chem 91, 1959-1967 (2019). Doi: 10.1515/pac-2019-0801.

9. Leach, M. R. Internet database of periodic tables. URL https://www.meta-synthesis.com/webbook/35_pt/pt_database.php.

10. Pyykkö, P. A suggested periodic table up to $\mathrm{Z} \leq 172$, based on Dirac-Fock calculations on atoms and ions. Phys. Chem. Chem. Phys. 13, 161-168 (2011). URL 10.1039/COCP01575J.

11. Giuliani, S. A. et al. Colloquium: Superheavy elements: Oganesson and beyond. Rev. Mod. Phys. 91, 011001 (2019). URL https://link.aps.org/doi/10.1103/RevModPhys.91.011001.

12. Nazarewicz, W. The limits of nuclear mass and charge. Nature Phys. 14, 537-541 (2018). URL https://doi.org/10.1038/s41567-018-0163-3. 
13. Pauling, L. The Nature of the Chemical Bond (Cornell University Press Ithaca, NY, 1960).

14. Ruedenberg, K. The physical nature of the chemical bond. Rev. Mod. Phys. 34, 326 (1962).

15. Frenking, G. \& Shaik, S. The chemical bond (Wiley Online Library, 2014).

16. Bacskay, G. B., Nordholm, S. \& Ruedenberg, K. The virial theorem and covalent bonding. J. Phys. Chem. A 122, 7880-7893 (2018).

17. Zhao, L.-L., Schwarz, W. H. E. \& Frenking, G. The Lewis electron-pair bonding model: the physical background one century later. Nature Revs. Chem. 3, 35-47 (2019).

18. Pauli, W. The connection between spin and statistics. Phys. Rev. 58, 716-722 (1940). URL https://link.aps.org/doi/10.1103/PhysRev.58.716.

19. Zhao, L., Pan, S., Holzmann, N., Schwerdtfeger, P. \& Frenking, G. Chemical bonding and bonding models of main-group compounds. Chem. Rev. 119, 8781-8845 (2019).

20. Goidenko, I. A. QED corrections for the valence electron in the heavy and superheavy metal atoms from the 11 and 12 groups. Eur. Phys. J. D 55, 35-42 (2009).

21. Goidenko, I., Labzowsky, L., Eliav, E., Kaldor, U. \& Pyykkö, P. QED corrections to the binding energy of the eka-radon $(Z=118)$ negative ion. Phys. Rev. A 67, 020102 (2003). URL https://link.aps.org/doi/10.1103/PhysRevA.67.020102. 
22. Thierfelder, C. \& Schwerdtfeger, P. Quantum electrodynamic corrections for the valence shell in heavy many-electron atoms. Phys. Rev. A 82, 062503 (2010). URL http://link.aps.org/doi/10.1103/PhysRevA.82.062503.

23. Slater, J. C. Atomic shielding constants. Phys. Rev. 36, 57-64 (1930). URL https://link.aps.org/doi/10.1103/PhysRev.36.57.

24. Zener, C. Analytic atomic wave functions. Phys. Rev. 36, 51-56 (1930). URL https://link.aps.org/doi/10.1103/PhysRev.36.51.

25. Fermi, E. Anomalous groups in the periodic system of elements. Nature 121, 502 (1928).

26. Ivanenko, D. D. \& Larin, S. Theory of the Periodic System of the Elements, vol. 2 (US Atomic Energy Commission, Technical Information Service, 1953).

27. Landau, L. D. \& Lifshitz, E. M. Quantum mechanics: non-relativistic theory, vol. 3 (2nd edition, Pergamon Press, Oxford, 1965).

28. Essén, H. Periodic table of the elements and the ThomasFermi atom. Int. J. Quant. Chem. 21, 717-726 (1982). URL https://onlinelibrary.wiley.com/doi/abs/10.1002/qua. 560210406. https://onlinelibrary.wiley.com/doi/pdf/10.1002/qua. 560210406.

29. Hartree, D. R. Variation of atomic wave functions with atomic number. Rev. Mod. Phys. 30, 63-68 (1958). URL https://link.aps.org/doi/10.1103/RevModPhys.30.63. 
30. Pyykkö, P. The physics behind chemistry, and the periodic table. Chem. Rev. 112, 371-384 (2012).

31. Mazurs, E. Graphic Representations of the Periodic Table During One Hundred Years (University of Alabama, University, Alabama, 1974).

32. Bensaude-Vincent, B. Graphic representations of the periodic system of chemical elements. In Tools and modes of representation in the laboratory sciences, 133-161 (Springer, Berlin, 2001).

33. Goudsmit, S. \& Richards, P. I. The order of electron shells in ionized atoms. Proceedings of the National Academy of Sciences of the United States of America 51, 664 (1964).

34. Madelung, E. Die Matematischen Hilfsmittel des Physikers (3rd. ed., Springer, Berlin, Berlin, 1936).

35. Janet, C. Concordance de l'arrangement quantique, de base, des électrons planétaires des atomes, avec la classification scalariforme, hélicö̈dale, des élements chimiques (Bauvais Imprimerie Départementale de l'Oise, Bauvais, 1930).

36. Sommerfeld, A. Electronic structure of the atom and the quantum theory. Memoirs and Proc. of the Manchester Lit. and Phil. Soc. 70(14), 141-151 (1925). See p. 144 for a 'Madelung rule'.

37. Allen, L. C. \& Knight, E. T. The löwdin challenge: Origin of the $n+1, n$ (Madelung) rule for filling the orbital configurations of the periodic table. Int. J. Quantum Chem. 90, 
80-88 (2002). URL https://onlinelibrary.wiley.com/doi/abs/10.1002/qua. 965. https://onlinelibrary.wiley.com/doi/pdf/10.1002/qua. 965.

38. Nefedov, V. I., Trzhaskovskaya, M. B. \& Yarzhemskii, V. G. Electronic configurations and the periodic table for superheavy elements. Doklady Physical Chemistry 408, 149151 (2006). URL https://doi.org/10.1134/S0012501606060029.

39. Laing, M. A revised periodic table: With the lanthanides repositioned. Foundations of Chemistry 7, 203 (2005). URL https://doi.org/10.1007/s10698-004-5959-9.

40. Scerri, E. R. \& Parsons, W. What elements belong in group 3 of the periodic table. In Mendeleev to Oganesson: A multidiciplinary perspective on the periodic table, 140-151 (Oxford University Press, Oxford, 2018).

41. Xu, W.-H. \& Pyykkö, P. Is the chemistry of lawrencium peculiar? Phys. Chem. Chem. Phys. 18, 17351-17355 (2016). Doi: 10.1039/C6CP02706G.

42. Steinhauser, G. Wohin mit dem f-block? Nachr. Chemie 67, 8-11 (2019). URL https://onlinelibrary.wiley.com/doi/abs/10.1002/nadc.20194086536. https://onlinelibrary.wiley.com/doi/pdf/10.1002/nadc. 20194086536.

43. Eichler, R. The periodic table - an experimenter's guide to transactinide chemistry. Radiochim. Acta 107, 865-877 (2019).

44. Cao, C.-S., Hu, H.-S., Li, J. \& Schwarz, W. H. E. Physical origin of chemical periodicities in the system of elements. Pure. Appl. Chem. 91, 1969-1999 (2019). URL https://doi.org/10.1515/pac-2019-0901. 
45. Grupen, C. Big Bang Nucleosynthesis, 339-355 (Springer International Publishing, Cham, 2020).

46. Moreno, D. et al. Re-examination of the $\mathrm{C}_{6} \mathrm{Li}_{6}$ structure: To be, or not to be symmetric. Chem. Europ. J. 19, 12668-12672 (2013). URL https://onlinelibrary.wiley.com/doi/abs/10.1002/chem. 201301460. https://onlinelibrary.wiley.com/doi/pdf/10.1002/chem. 201301460.

47. Drozdov, A., Eremets, M., Troyan, I., Ksenofontov, V. \& Shylin, S. Conventional superconductivity at 203 Kelvin at high pressures in the sulfur hydride system. Nature 525, $73(2015)$.

48. Somayazulu, M. et al. Evidence for superconductivity above $260 \mathrm{~K}$ in lanthanum superhydride at megabar pressures. Phys. Rev. Lett. 122, 027001 (2019). URL https://link.aps.org/doi/10.1103/PhysRevLett.122.027001.

49. Drozdov, A. et al. Superconductivity at $250 \mathrm{~K}$ in lanthanum hydride under high pressures. Nature 569, 528-531 (2019).

50. Loubeyre, P., Occelli, F. \& Dumas, P. Synchrotron infrared spectroscopic evidence of the probable transition to metal hydrogen. Nature 577, 631-635 (2020).

51. Drake, G. \& Martin, W. C. Ionization energies and quantum electrodynamic effects in the lower 1sns and 1snp levels of neutral helium (4he i). Canadian journal of physics 76, 679 (1998). 
52. Hotokka, M., Kindstedt, T., Pyykkö, P. \& Roos, B. O. On bonding in transition-metal helide ions. Mol. Phys. 52, 23-32 (1984).

53. Wesendrup, R., Pernpointner, M. \& Schwerdtfeger, P. Coulomb-stable triply charged diatomic: HeY ${ }^{3+}$. Phys. Rev. A 60, R3347-R3349 (1999). URL https://link.aps.org/doi/10.1103/PhysRevA.60.R3347.

54. Wright, T. G., Lee, E. P. F., Hotokka, M. \& Pyykkö, P. Al ${ }^{3+}-$ He: stability and spectroscopy. Chem. Phys. Lett. 392, 281-283 (2004).

55. Dong, X. et al. A stable compound of helium and sodium at high pressure. Nature Chem. 9, 440-445 (2017).

56. Pyykkö, P. Dirac-Fock one-centre calculations part 8. The $1 \sigma$ states of $\mathrm{ScH}$, YH, LaH, AcH, TmH, LuH and LrH. Phys. Scr. 20, 647-651 (1979). URL https : //doi.org/10.1088\%2F0031-8949\%2F20\%2F5-6\%2F016.

57. Shchukarev, S. Periodicheskaya sistema di mendeleeva i sovremennaya khimiya. Periodicheskii Zakon i Stroenie Atoma," Atomisdat, Moscow (1971).

58. Imyanitov, N. S. Does the period table appear doubled? two variants of division of elements into two subsets. internal and secondary periodicity. Foundations of Chemistry 21, 255-284 (2019).

59. Trinquier, G., Malrieu, J.-P. \& Daudey, J.-P. Ab initio study of the regular polyhedral molecules $\mathrm{N}_{4}, \mathrm{P}_{4}, \mathrm{As}_{4}, \mathrm{~N}_{8}, \mathrm{P}_{8}$ and $\mathrm{As}_{8}$. Chem. Phys. Lett. 80, 552-557 (1981). 
60. Kutzelnigg, W. Chemical bonding in higher main group elements. Angew. Chem. Int. Ed. 23, 272-295 (1984). URL https://onlinelibrary.wiley.com/doi/abs/10.1002/anie.198402721. https://onlinelibrary.wiley.com/doi/pdf/10.1002/anie.198402721.

61. Düllmann, C. E. et al. Chemical investigation of hassium (element 108). Nature 418, 859 (2002).

62. Lu, J.-B. et al. Experimental and theoretical identification of the fe(vii) oxidation state in $\mathrm{FeO}_{4}^{-}$. Phys. Chem. Chem. Phys. 18, 31125-31131 (2016). URL http://dx.doi.org/10.1039/C6CP06753K.

63. Autschbach, J., Siekierski, S., Seth, M., Schwerdtfeger, P. \& Schwarz, W. H. E. Dependence of relativistic effects on electronic configuration in the neutral atoms of $d$ - and f-block elements. J. Comput. Chem. 23, 804-813 (2002). URL https://onlinelibrary.wiley.com/doi/abs/10.1002/jcc. 10060.

64. Mann, J. B., Meek, T. L., Knight, E. T., Capitani, J. F. \& Allen, L. C. Configuration energies of the d-block elements. J. Am. Chem. Soc. 122, 5132-5137 (2000).

65. Allen, L. C. Extension and completion of the periodic table. J. Am. Chem. Soc. 114, 1510-1511 (1992).

66. Nyholm, R. Electron configuration and structure of transition-metal complexes. Tilden lecture. Proce. Chem. Soc. 1961, 273-298 (1961). 
67. Kaupp, M. The role of radial nodes of atomic orbitals for chemical bonding and the periodic table. Journal of Computational Chemistry 28, 320-325 (2007). URL https://onlinelibrary.wiley.com/doi/abs/10.1002/jcc. 20522. https://onlinelibrary.wiley.com/doi/pdf/10.1002/jcc. 20522.

68. Newell, D. B. et al. The codata 2017 values of $\mathrm{h}, \mathrm{e}, \mathrm{k}$, and na for the revision of the si. Metrologia 55, L13 (2018).

69. Pašteka, L. F., Eliav, E., Borschevsky, A., Kaldor, U. \& Schwerdtfeger, P. Relativistic coupled cluster calculations with variational quantum electrodynamics resolve the discrepancy between experiment and theory concerning the electron affinity and ionization potential of gold. Phys. Rev. Lett. 118, 023002 (2017). URL https://link.aps.org/doi/10.1103/PhysRevLett.118.023002.

70. Rose, S. J., Grant, I. P. \& Pyper, N. C. The direct and indirect effects in the relativistic modification of atomic valence orbitals. J. Phys. B 11, 1171-1176 (1978).

71. Pyykkö, P. \& Desclaux, J. P. Relativity and the periodic system of elements. Acc. Chem. Res. 12, 276-281 (1979). URL http://dx.doi.org/10.1021/ar50140a002.

72. Pyykkö, P. Relativistic effects in structural chemistry. Chem. Rev. 88, 563-594 (1988). URL http://pubs.acs.org/doi/abs/10.1021/cr00085a006.

73. Pyykkö, P. Relativistic effects in chemistry: more common than you thought. Annual Rev. Phys. Chem. 63, 45-64 (2012). 
74. Schwarz, W. H. E., van Wezenbeek, E. M., Baerends, E. J. \& Snijders, J. G. The origin of relativistic effects of atomic orbitals. J. Phys. B 22, 1515-1530 (1989). URL http://stacks .iop.org/0953-4075/22/i=10/a=008.

75. Dehmer, J. L. Phase-amplitude method in atomic physics. II. Z dependence of spinorbit coupling. Phys. Rev. A 7, 4-9 (1973).

76. Mayers, D. F. Relativistic self-consistent field calculation for mercury. Proc. Roy. Soc. (London) A 241, 93-109 (1957).

77. Jerabek, P., Schuetrumpf, B., Schwerdtfeger, P. \& Nazarewicz, W. Electron and nucleon localization functions of oganesson: Approaching the Thomas-Fermi limit. Phys. Rev. Lett. 120, 053001 (2018). URL https://link.aps.org/doi/10.1103/PhysRevLett.120.053001.

78. Desclaux, J. Relativistic Dirac-Fock expectation values for atoms with $Z=$ 1 to $Z=120 . \quad$ At. Data Nucl. Data Tabl. 12, $311-406$ (1973). URL http://www.sciencedirect.com/science/article/pii/0092640X7390020X.

79. Schwerdtfeger, P. \& Lein, M. Theoretical chemistry of gold - from atoms to molecules, clusters, surfaces and the solid state. In Mohr, F. (ed.) Gold Chemistry: Applications and Future Directions in the Life Sciences, 183-247 (Wiley-VCH, Weinheim, 2009). URL http://dx.doi.org/10.1002/9783527626724.ch4. 
80. Dyall, K., Grant, I., Johnson, C., Parpia, F. \& Plummer, E. Grasp: A general-purpose relativistic atomic structure program. Comput. Phys. Commun. 55, 425-456 (1989). URL http://www.sciencedirect.com/science/article/pii/0010465589901367.

81. Türler, A. \& Pershina, V. Advances in the production and chemistry of the heaviest elements. Chem. Rev. 113, 1237-1312 (2013). URL http://pubs.acs .org/doi/abs/10.1021/cr3002438.

82. Eliav, E., Kaldor, U., Schwerdtfeger, P., Hess, B. A. \& Ishikawa, Y. Ground state electron configuration of element 111. Phys. Rev. Lett. 73, 3203-3206 (1994). URL http://link.aps.org/doi/10.1103/PhysRevLett.73.3203.

83. Seth, M., Schwerdtfeger, P. \& Dolg, M. The chemistry of the superheavy elements. I. Pseudopotentials for 111 and 112 and relativistic coupled cluster calculations for (112) $\mathrm{H}^{+},(112) \mathrm{F}_{2}$, and $(112) \mathrm{F}_{4}$. J. Chem. Phys. 106, 3623-3632 (1997). URL https://doi.org/10.1063/1.473437.

84. Seth, M., Cooke, F., Schwerdtfeger, P., Heully, J.-L. \& Pelissier, M. The chemistry of the superheavy elements. ii. the stability of high oxidation states in group 11 elements: Relativistic coupled cluster calculations for the di-, tetra- and hexafluoro metallates of $\mathrm{Cu}, \mathrm{Ag}, \mathrm{Au}$, and element 111. J. Chem. Phys. 109, 3935-3943 (1998). URL https://doi.org/10.1063/1.476993.

85. Schwerdtfeger, P., Dolg, M., Schwarz, W. H. E., Bowmaker, G. A. \& Boyd, P. D. W. Relativistic effects in gold chemistry. I. diatomic gold compounds. J. 
Chem. Phys. 91, 1762-1774 (1989). URL https://doi.org/10.1063/1.457082. https://doi.org/10.1063/1.457082.

86. Söhnel, T., Hermann, H. \& Schwerdtfeger, P. Towards the understanding of solid-state structures: From cubic to chainlike arrangements in group 11 halides. Angew. Chem. Int. Ed. 40, 4381-4385 (2001).

87. Pahl, E. \& Schwerdtfeger, P. Mercury: From atoms to solids. In Sattler, K. D. (ed.) Handbook of nanophysics: clusters and fullerenes, chap. 3, 1-13 (CRC press, Boca Raton, 2010).

88. Calvo, F., Pahl, E., Wormit, M. \& Schwerdtfeger, P. Evidence for low-temperature melting of mercury owing to relativity. Angew. Chem. Int. Ed. 52, 7583-7585 (2013). URL http://dx.doi.org/10.1002/anie.201302742.

89. Steenbergen, K. G., Pahl, E. \& Schwerdtfeger, P. Accurate, large-scale density functional melting of $\mathrm{Hg}$ : Relativistic effects decrease melting temperature by 160 K. J. Phys. Chem. Lett. 8, 1407-1412 (2017). URL https://doi.org/10.1021/acs.jpclett.7b00354.

90. Mewes, J.-M., Smits, O. R., Kresse, G. \& Schwerdtfeger, P. Copernicium: A relativistic noble liquid. Angew. Chem. Int. Ed. 58, 17964-17968 (2019). URL https://onlinelibrary.wiley.com/doi/abs/10.1002/anie. 201906966. https://onlinelibrary.wiley.com/doi/pdf/10.1002/anie.201906966. 
91. Pitzer, K. S. Are elements 112, 114, and 118 relatively inert gases? J. Chem. Phys. 63, 1032-1033 (1975). URL http://scitation.aip.org/content/aip/journal/jcp/63/2/10.1063/1.431398.

92. Gaston, N., Opahle, I., Gäggeler, H. W. \& Schwerdtfeger, P. Is eka-mercury (element 112) a group 12 metal? Angew. Chem. Int. Ed. 46, 1663-1666 (2007). URL http://dx.doi.org/10.1002/anie.200604262.

93. Deng, S., Simon, A. \& Köhler, J. Superconductivity and chemical bonding in mercury. Angew. Chem. Int. Ed. 37, 640-643 (1998).

94. Kaupp, M. \& von Schnering, H. G. Gaseous mercury (IV) fluoride, $\mathrm{HgF}_{4}$ : an ab initio study. Angew. Chem. Int. Ed. 32, 861-863 (1993).

95. Wang, X., Andrews, L., Riedel, S. \& Kaupp, M. Mercury is a transition metal: the first experimental evidence for $\mathrm{HgF}_{4}$. Angew. Chem. Int. Ed. 46, 8371-8375 (2007).

96. Eichler, R. et al. Chemical characterization of element 112. Nature 447, 72 (2007).

97. Trombach, L., Ehlert, S., Grimme, S., Schwerdtfeger, P. \& Mewes, J.-M. Exploring the chemical nature of super-heavy main-group elements by means of efficient plane-wave density-functional theory. Phys. Chem. Chem. Phys. 21, 18048-18058 (2019). URL http://dx.doi.org/10.1039/C9CP02455G.

98. Schwarz, W. H. E. An introduction to relativistic quantum chemistry. In Barysz, M. \& Ishikawa, M. (eds.) Relativistic methods for chemists, 1-62 (Springer, Dordrecht, 2010). 
99. Froben, F., Schulze, W. \& Kloss, U. Raman spectra of matrix-isolated group iiia dimers: $\mathrm{Ga}_{2}, \mathrm{In}_{2}, \mathrm{Tl}_{2}$. Chem. Phys. Lett. 99, 500 - 502 (1983). URL http://www.sciencedirect.com/science/article/pii/0009261483801833.

100. Liu, W., van Wüllen, C., Wang, F. \& Li, L. Spectroscopic constants of and $\left.\mathrm{M}_{2} \mathrm{M}=\mathrm{Tl}, \mathrm{E} 113, \mathrm{Bi}, \mathrm{E} 115\right)$ : Direct comparisons of four- and twocomponent approaches in the framework of relativistic density functional theory. $J$. Chem. Phys. 116, 3626-3634 (2002). URL https://doi.org/10.1063/1.1446026. https://doi.org/10.1063/1.1446026.

101. Pershina, V. Relativity in the electronic structure of the heaviest elements and its influence on periodicities in properties. Radiochim. Acta 107, 833-863 (2019).

102. Hermann, A., Furthmüller, J., Gäggeler, H. W. \& Schwerdtfeger, P. Spin-orbit effects in structural and electronic properties for the solid state of the group-14 elements from carbon to superheavy element 114. Phys. Rev. B 82, 155116 (2010). URL https://link.aps.org/doi/10.1103/PhysRevB.82.155116.

103. Eichler, R. et al. Indication for a volatile element 114. Radiochim. Acta 98, 133-139 (2010).

104. Yakushev, A. et al. Superheavy element flerovium (element 114) is a volatile metal. Inorg. Chem. 53, 1624-1629 (2014). URL https://doi.org/10.1021/ic4026766. PMID: 24456007, https://doi.org/10.1021/ic4026766. 
105. Egdell, R. G., Hotokka, M., Laaksonen, L., Pyykkö, P. \& Snijders, J. G. Photoelectron spectra and their relativistic interpretation for gaseous bismuth trihalides. Chem. Phys. $72,237-247(1982)$

106. Walsh, J. P. S. \& Freedman, D. E. High-pressure synthesis: A new frontier in the search for next-generation intermetallic compounds. Acc. Chem. Res. 51, 1315-1323 (2018).

107. Nash, C. S. Atomic and molecular properties of elements 112, 114, and 118. J. Phys. Chem. A 109, 3493-3500 (2005).

108. Nash, C. S. \& Bursten, B. E. Spin-orbit coupling versus the VSEPR method: On the possibility of a nonplanar structure for the super-heavy noble gas tetrafluoride (118) $\mathrm{F}_{4}$. Angew. Chem. Int. Ed. 38, 151-153 (1999).

109. Jerabek, P., Smits, O. R., Mewes, J.-M., Peterson, K. A. \& Schwerdtfeger, P. Solid oganesson via a many-body interaction expansion based on relativistic coupled-cluster theory and from plane-wave relativistic density functional theory. J. Phys. Chem. A 123, 4201-4211 (2019). URL https://doi .org/10.1021/acs.jpca.9b01947. PMID: 31017443, https://doi.org/10.1021/acs.jpca.9b01947.

110. Mewes, J.-M., Jerabek, P., Smits, O. R. \& Schwerdtfeger, P. Oganesson is a semiconductor: On the relativistic band-gap narrowing in the heaviest noble-gas solids. Angew. Chem. Int. Ed. 58, 14260-14264 (2019). URL https://onlinelibrary.wiley.com/doi/abs/10.1002/anie.201908327. https://onlinelibrary.wiley.com/doi/pdf/10.1002/anie. 201908327. 
111. Eliav, E., Kaldor, U., Ishikawa, Y. \& Pyykkö, P. Element 118: The first rare gas with an electron affinity. Phys. Rev. Lett. 77, 5350-5352 (1996). URL https://link.aps.org/doi/10.1103/PhysRevLett.77.5350.

112. Sidgwick, N. V. The covalent link in chemistry. Cornell Univ. Press, Ithaca, NY (1933).

113. Sidgwick, N. \& Powell, H. Bakerian lecture. stereochemical types and valency groups. Proc. Royal Soc. A 176, 153-180 (1940).

114. Schwerdtfeger, P., Heath, G. A., Dolg, M. \& Bennett, M. A. Low valencies and periodic trends in heavy element chemistry. a theoretical study of relativistic effects and electron correlation effects in group 13 and period 6 hydrides and halides. J. Am. Chem. Soc. 114, 7518-7527 (1992). URL https://doi.org/10.1021/ja00045a027.

115. Seth, M., Faegri, K. \& Schwerdtfeger, P. The stability of the oxidation state +4 in group 14 compounds from carbon to element 114. Angew. Chem. Int. Ed. 37, 24932496 (1998).

116. Schwerdtfeger, P. \& Seth, M. Relativistic quantum chemistry of the superheavy elements. closed-shell element 114 as a case study. J. Nucl. Radiochem. Sci. 3, 133-136 (2002).

117. Vest, B., Klinkhammer, K., Thierfelder, C., Lein, M. \& Schwerdtfeger, P. Kinetic and thermodynamic stability of the group 13 trihydrides. Inorg. Chem. 48, 7953-7961 (2009). URL https://doi.org/10.1021/ic900997p. PMID: 19601590, https://doi.org/10.1021/ic900997p. 
118. Ahuja, R., Blomqvist, A., Larsson, P., Pyykkö, P. \& Zaleski-Ejgierd, P. Relativity and the lead-acid battery. Phys. Rev. Lett. 106, 018301 (2011).

119. Roos, B. O. \& Pyykkö, P. Bonding trends in molecular compounds of lanthanides: The double-bonded carbene cations $\mathrm{LnCH}_{2}^{+}, \mathrm{Ln}=\mathrm{Sc}, \mathrm{Y}, \mathrm{La}-\mathrm{Lu}$. Chem. Eur. J. 16, $270-275(2010)$.

120. Xu, W.-H. et al. Rare-earth monocarbonyls MCO: comprehensive infrared observations and a transparent theoretical interpretation for $\mathrm{M}=\mathrm{Sc}$; Y; La-Lu. Chem. Sci. 3, 15481554 (2012).

121. Goldschmidt, V. M., Barth, T. \& Lunde, G. Geochemische Verteilungsgesetze der Elemente. V. Isomorphie und Polymorphie der Sesquioxyde. Die Lanthaniden-Kontraktion und Ihre Konsequenzen. Skrifter Norske Videnskaps-Akad. Oslo, I: Mat. Naturv. Kl. 7, 1-59 (1925).

122. Gao, C. et al. Observation of the asphericity of 4 f-electron density and its relation to the magnetic anisotropy axis in single-molecule magnets. Nature Chemistry 1-7 (2019).

123. Ryan, A. J. et al. Synthesis, structure, and magnetism of tris(amide) $\left[\operatorname{lnN}\left(\mathrm{SiMe}_{3}\right)_{23}\right]^{-1}$ complexes of the non-traditional +2 lanthanide ions. Chem. Eur. J. 24, 7702-7709 (2018).

124. Kaltsoyannis, N., Hay, P. J., Li, J., Blaudeau, J.-P. \& Bursten, B. E. Theoretical studies of the electronic structure of compounds of the actinide elements. In Morss, L. R., 
Edelstein, N. M. \& Fuger, J. (eds.) The Chemistry of the Actinide and Transactinide Elements, Third Edition, vol. 3, 1893-2012 (Springer, Dordrecht, 2005).

125. Galley, S. S. et al. Synthesis and characterization of tris-chelate complexes for understanding f-orbital bonding in later actinides. J. Am. Chem. Soc. 141, 2356-2366 (2019).

126. White, F. D., Dan, D. \& Albrecht-Schmitt, T. E. Contemporary chemistry of berkelium and californium. Chem. Eur. J. 25, 10251-10261 (2019).

127. Vitova, T. et al. The role of the $5 \mathrm{f}$ valence orbitals of early actinides in chemical bonding. Nature Commun. 8, 16053 (2017).

128. Seth, M., Dolg, M., Fulde, P. \& Schwerdtfeger, P. Lanthanide and actinide contractions: relativistic and shell structure effects. J. Am. Chem. Soc. 117, 6597-6598 (1995).

129. Küchle, W., Dolg, M. \& Stoll, H. Ab initio study of the lanthanide and actinide contraction. J. Phys. Chem. A 101, 7128-7133 (1997). URL https://doi.org/10.1021/jp970920c. https://doi.org/10.1021/jp970920c.

130. Chemey, A. T. \& Albrecht-Schmitt, T. E. Evolution of the periodic table through the synthesis of new elements. Radiochim. Acta 107, 771-801 (2019).

131. Pyykkö, P. Is the periodic table all right ("pt ok")? In EPJ Web of Conferences, vol. 131, 01001 (EDP sciences, 2016). 
132. Münzenberg, G. From bohrium to copernicium and beyond SHE research at SHIP. Nucl. Phys. A 944, 5 - 29 (2015). URL http://www.sciencedirect.com/science/article/pii/S0375947415001372.

133. Itkis, M., Vardaci, E., Itkis, I., Knyazheva, G. \& Kozulin, E. Fusion and fission of heavy and superheavy nuclei (experiment). Nucl. Phys. A 944, $204-237$ (2015). URL http://www.sciencedirect.com/science/article/pii/S0375947415002067.

134. Morita, K. SHE research at RIKEN/GARIS. Nucl. Phys. A 944, $30-61$ (2015). URL http://www.sciencedirect.com/science/article/pii/S0375947415002304.

135. Dmitriev, S., Itkis, M. \& Oganessian, Y. Status and perspectives of the Dubna superheavy element factory. EPJ Web Conf. 131, 08001 (2016). URL 10.1051/epjconf/201613108001.

136. Ball, P. Extreme chemistry: experiments at the edge of the periodic table. Nature $\mathbf{5 6 5}$, 552 (2019).

137. Lim, I. S. et al. Relativistic coupled-cluster static dipole polarizabilities of the alkali metals from Li to element 119. Phys. Rev. A 60, 2822-2828 (1999). URL https://link.aps.org/doi/10.1103/PhysRevA.60.2822.

138. Schwerdtfeger, P. Relativistic effects in molecular structure of s-and p-block elements. In Domenicano, A. \& Hargittai, I. (eds.) Strength from Weakness: Structural Consequences of Weak Interactions in Molecules, Supermolecules, and Crystals, 169-190 (NATO Science Series, Kluwer, Dordrecht, 2002). 
139. Borschevsky, A., Pershina, V., Eliav, E. \& Kaldor, U. Ab initio studies of atomic properties and experimental behavior of element 119 and its lighter homologs. $J$. Chem. Phys. 138, 124302 (2013). URL https://doi.org/10.1063/1.4795433. https://doi.org/10.1063/1.4795433.

140. Demidov, Y. A. \& Zaitsevskii, A. V. A comparative study of the chemical properties of element 120 and its homologs. Radiochem. 55, 461-465 (2013). URL https://doi.org/10.1134/S1066362213050019.

141. Seaborg, G. T. Prospects for further considerable extension of the periodic table. J. Chem. Ed. 46, 626-634 (1969).

142. Fricke, B., Greiner, W. \& Waber, J. T. The continuation of the periodic table up to $Z=172$. the chemistry of superheavy elements. Theoret. Chim. Acta 21, 235-260 (1971). URL 10.1007/BF01172015.

143. Indelicato, P., Bieroń, J. \& Jönsson, P. Are mcdf calculations $101 \%$ correct in the super-heavy elements range? Theoret. Chem. Acc. 129, 495-505 (2011).

144. Dognon, J.-P. \& Pyykkö, P. Chemistry of the $5 \mathrm{~g}$ elements: Relativistic calculations on hexafluorides. Angew. Chem. Int. Ed. 56, 10132-10134 (2017). URL https://onlinelibrary.wiley.com/doi/abs/10.1002/anie.201701609.

145. Schwerdtfeger, P., Pašteka, L. F., Punnett, A. \& Bowman, P. O. Relativistic and quantum electrodynamic effects in superheavy elements. Nucl. Phys. A 944, 551-577 (2015). 
146. Grant, I. P. Formulation of the relativistic N-electron problem. In Malli, G. L. (ed.) Relativistic Effects in Atoms, Molecules, and Solids, 73-88 (Plenum Press, New York, 1983).

147. Thaller, B. The Dirac equation (Springer, Berlin, 1992).

148. Pomeranchuk, I. Y. \& Smorodinsky, Y. A. On the energy levels of systems with $Z>137$. J. Fiz. USSR 9, 97-100 (1945).

149. Zeldovich, Y. B. \& Popov, V. S. Electronic structure of superheavy atoms. Soviet Physics Uspekhi 14, 673-694 (1972). URL https://doi .org/10.1070\%2Fpu1972v014n06abeh004735.

150. Reinhardt, J. \& Greiner, W. Quantum electrodynamics of strong fields. Rep. Prog. Phys. 40, 219-295 (1977). URL https://doi.org/10.1088\%2F0034-4885\%2F40\%2F3\%2F001.

151. Maltsev, I. A. et al. How to observe the vacuum decay in lowenergy heavy-ion collisions. Phys. Rev. Lett. 123, 113401 (2019). URL https://link.aps.org/doi/10.1103/PhysRevLett.123.113401.

152. Oberhummer, H., Csoto, A. \& Schlattl, H. Stellar production rates of carbon and its abundance in the universe. Science 289, 88-90 (2000).

153. Oberhummer, H., Csótó, A. \& Schlattl, H. Fine-tuning carbon-based life in the universe by the triple-alpha process in red giants. In The future of the universe and the future of our civilization, 197-205 (World Scientific, 2000). 
154. Borsanyi, S. et al. Ab initio calculation of the neutronproton mass difference. Science $\mathbf{3 4 7}, \quad 1452-1455 \quad$ (2015). URL https://science.sciencemag.org/content/347/6229/1452. https://science.sciencemag. org/content/347/6229/1452.full.pdf.

155. Barrow, J. D. Impossibility: The limits of science and the science of limits (Oxford University Press on Demand, 1999).

156. Uzan, J.-P. The fundamental constants and their variation: observational and theoretical status. Rev. Mod. Phys. 75, 403-455 (2003). URL https://link.aps.org/doi/10.1103/RevModPhys.75.403.

157. Pašteka, L. c. v. F., Hao, Y., Borschevsky, A., Flambaum, V. V. \& Schwerdtfeger, P. Material size dependence on fundamental constants. Phys. Rev. Lett. 122, 160801 (2019). URL https://link.aps.org/doi/10.1103/PhysRevLett.122.160801.

158. Guggenheimer, K. Remarques sur la constitution des noyaux - II. J. Phys. Radium 5, 475-485 (1934). URL https://doi.org/10.1051/jphysrad:0193400509047500.

159. Guggenheimer, K. Remarques sur la constitution des noyaux atomiques - I. J. Phys. Radium 5, 253-256 (1934). URL https://doi.org/10.1051/jphysrad:0193400506025300.

160. Fea, G. Tabelle Riassuntive e Bibliografia Delle Trasmutazioni Artificiali. Il Nuovo Cimento (1924-1942) 12, 368-406 (1935). URL https://doi.org/10.1007/BF02958685. 
161. Segre, E. Nuclei and particles: an introduction to nuclear and subnuclear physics (Benjamin, New York, 1964).

162. Hollander, J. M., Perlman, I. \& Seaborg, G. T. Table of isotopes. Rev. Mod. Phys. 25, 469-651 (1953). URL https://link.aps.org/doi/10.1103/RevModPhys.25.469.

163. Strominger, D., Hollander, J. M. \& Seaborg, G. T. Table of isotopes. Rev. Mod. Phys. 30, 585-904 (1958). URL https://link.aps.org/doi/10.1103/RevModPhys .30 . 585.

164. Burbidge, E. M., Burbidge, G. R., Fowler, W. A. \& Hoyle, F. Synthesis of the elements in stars. Rev. Mod. Phys. 29, 547-650 (1957). URL https://link.aps.org/doi/10.1103/RevModPhys.29.547.

165. Schatz, H. et al. End point of the rp process on accreting neutron stars. Phys. Rev. Lett. 86, 3471-3474 (2001). URL https://link.aps.org/doi/10.1103/PhysRevLett.86.3471.

166. Pignatari, M., Goebel, K., Reifarth, R. \& Travaglio, C. The production of proton-rich isotopes beyond iron: The $\gamma$-process in stars. Int. J. Mod. Phys. E 25, 1630003 (2016).

167. Gamow, G. Expanding universe and the origin of elements. Phys. Rev. 70, 572 (1946).

168. Alpher, R. A. \& Herman, R. C. Theory of the origin and relative abundance distribution of the elements. Rev. Mod. Phys. 22, 153 (1950).

169. Cirgiliano, V. et al. Precision beta decay as a probe of new physics (2019). 1907.02164. 
170. Yue, A. T. et al. Improved determination of the neutron lifetime. Phys. Rev. Lett. 111, $222501 \quad$ (2013). URL https://link.aps.org/doi/10.1103/PhysRevLett.111.222501.

171. Ezhov, V. F. et al. Measurement of the neutron lifetime with ultracold neutrons stored in a magneto-gravitational trap. JETP Letters 107, 671-675 (2018). URL https://doi.org/10.1134/S0021364018110024.

172. Thielemann, F.-K., Eichler, M., Panov, I. \& Wehmeyer, B. Neutron star mergers and nucleosynthesis of heavy elements. Annual Review of Nuclear and Particle Science 67, $253-274$ (2017).

173. Frebel, A. From nuclei to the cosmos: Tracing heavy-element production with the oldest stars. Annual Review of Nuclear and Particle Science 68, 237-269 (2018).

174. Horowitz, C. J. et al. r-process nucleosynthesis: connecting rare-isotope beam facilities with the cosmos. J. Phys. G: Nucl. Part. Phys. 46, 083001 (2019). URL https://doi.org/10.1088\%2F1361-6471\%2Fab0849.

175. Heger, A., Hoffman, R. D., Rauscher, T. \& Woosley, S. E. Nucleosynthesis in massive stars - including all stable isotopes (2000). astro-ph/0006350.

176. Hampel, M., Stancliffe, R. J., Lugaro, M. \& Meyer, B. S. The intermediate neutroncapture process and carbon-enhanced metal-poor stars. Astrophys. J. 831, 171 (2016). URL https://doi.org/10.3847\%2F0004-637x\%2F831\%2F2\%2F171. 
177. Clarkson, O., Herwig, F. \& Pignatari, M. Pop III i-process nucleosynthesis and the elemental abundances of SMSS J0313-6708 and the most iron-poor stars. Monthly Not. Royal Astr. Soc.: Lett. 474, L37-L41 (2017). URL https://doi.org/10.1093/mnrasl/slx190. http://oup.prod.sis.lan/mnrasl/article-pdf/474/1/L37/24841490/slx190.pdf.

178. Busso, M., Gallino, R. \& Wasserburg, G. J. Nucleosynthesis in asymptotic giant branch stars: Relevance for galactic enrichment and solar system formation. Ann. Rev. Astron. Astrophys. 37, 239-309 (1999). URL https://doi.org/10.1146/annurev.astro.37.1.239. https://doi.org/10.1146/annurev. astro.37.1.239.

179. Cameron, A. G. Abundances of the elements in the solar system. Space Sci. Rev. 15, 121-146 (1973).

180. Ratzel, U. et al. Nucleosynthesis at the termination point of the $s$ process. Phys. Rev. C 70, 065803 (2004). URL https://link.aps.org/doi/10.1103/PhysRevC.70.065803.

181. Roederer, I. U. et al. New hubble space telescope observations of heavy elements in four metal-poor stars. Astrophys. J. Suppl. Ser. 203, 27 (2012).

182. Clayton, D. D. Principles of stellar evolution and nucleosynthesis (University of Chicago press, 1983).

183. Clayton, D. D., Fowler, W. A., Hull, T. \& Zimmerman, B. Neutron capture chains in heavy element synthesis. Annals Phys. 12, 331-408 (1961). 
184. Seeger, P. A., Fowler, W. A. \& Clayton, D. D. Nucleosynthesis of heavy elements by neutron capture. Astrophys. J. 121-166 (1965).

185. Arlandini, C. et al. Neutron capture in low-mass asymptotic giant branch stars: cross sections and abundance signatures. Astrophys. J. 525, 886 (1999).

186. Straniero, O., Gallino, R. \& Cristallo, S. s process in low-mass asymptotic giant branch stars. Nuclear Physics A 777, 311-339 (2006).

187. Cristallo, S. et al. Asymptotic-giant-branch models at very low metallicity. Publ. Astronom. Soc. Australia 26, 139-144 (2009).

188. Ulrich, R. Explosive nucleosynthesis, ed. DN Schramm, WD Arnett (Austin University of Texas Press 1973) p. 139 (1973).

189. Käppeler, F., Gallino, R., Bisterzo, S. \& Aoki, W. The s process: Nuclear physics, stellar models, and observations. Rev. Mod. Phys. 83, 157 (2011).

190. Schwarzschild, M. \& Härm, R. Hydrogen mixing by helium-shell flashes. Astrophys. J. 150, 961 (1967).

191. Gallino, R. et al. Evolution and nucleosynthesis in low-mass asymptotic giant branch stars. ii. neutron capture and the s-process. Astrophys. J. 497, 388 (1998).

192. Peters, J. G. Nucleosynthesis by the s-process in stars of 9 and 15 solar masses. Astrophys. J. 154, 225 (1968). 
193. Travaglio, C. et al. Galactic chemical evolution of heavy elements: From barium to europium. Astrophys. J. 521, 691 (1999).

194. Travaglio, C. et al. Galactic evolution of Sr, Y, and Zr: a multiplicity of nucleosynthetic processes. Astrophys. J. 601, 864 (2004).

195. Siegel, D. M., Barnes, J. \& Metzger, B. D. Collapsars as a major source of r-process elements. Nature 569, 241-244 (2019). URL https://doi.org/10.1038/s41586-019-1136-0.

196. Argast, D., Samland, M., Thielemann, F.-K. \& Qian, Y.-Z. Neutron star mergers versus core-collapse supernovae as dominant r-process sites in the early galaxy. Astron. Astrophys. 416, 997-1011 (2004).

197. Abbott, B. P. et al. Multi-messenger observations of a binary neutron star merger. Astrophys. J. Lett 848, L12 (2017).

198. Pian, E. et al. Spectroscopic identification of r-process nucleosynthesis in a double neutron-star merger. Nature 551, 67 (2017).

199. Bartos, I. \& Marka, S. A nearby neutron-star merger explains the actinide abundances in the early solar system. Nature 569, 85 (2019).

200. Abbott, B. P. et al. GW170817: observation of gravitational waves from a binary neutron star inspiral. Phys. Rev. Lett. 119, 161101 (2017). 
201. Cowperthwaite, P. et al. The electromagnetic counterpart of the binary neutron star merger ligo/virgo gw170817. ii. uv, optical, and near-infrared light curves and comparison to kilonova models. Astrophys. J. Lett. 848, L17 (2017).

202. Holmbeck, E. M. et al. Actinide production in the neutron-rich ejecta of a neutron star merger. Astrophys. J. 870, 23 (2018). URL https://doi.org/10.3847\%2F1538-4357\%2Faaefef.

203. Watson, D. et al. Identification of strontium in the merger of two neutron stars. Nature 574, 497-500 (2019). URL https://doi.org/10.1038/s41586-019-1676-3.

204. Truran, J. W. Nucleosynthesis. Ann. Rev. Nucl. Part. Sci. 34, 53-97 (1984).

205. Wallerstein, G. et al. Synthesis of the elements in stars: forty years of progress. Rev. Mod. Phys. 69, 995 (1997).

206. Cheifetz, E., Jared, R. C., Giusti, E. R. \& Thompson, S. G. Search for superheavy elements in nature. Phys. Rev. C 6, 1348-1361 (1972). URL https://link.aps.org/doi/10.1103/PhysRevC.6.1348.

207. Schramm, D. N. Implied superheavy element decay lifetime from meteorites. Nature 233, 258 (1971).

208. Köber, E. \& Langrock, E. J. Search for superheavy elements in the nature. Isotopenpraxis Isotopes in Environmental and Health Studies 26, 576-583 (1990). URL https://doi.org/10.1080/10256019008622438. https://doi.org/10.1080/10256019008622438. 
209. Ter-Akopian, G. M. \& Dmitriev, S. N. Searches for superheavy elements in nature: Cosmic-ray nuclei; spontaneous fission. Nucl. Phys. A 944, 177 - 189 (2015). URL http://www.sciencedirect.com/science/article/pii/S0375947415002031.

210. Petermann, I. et al. Have superheavy elements been produced in nature? Eur. Phys. J. A 48, 122 (2012). URL http://link.springer.com/10.1140/epja/i2012-12122-6. 1207.3432.

211. Goriely, S. \& Pinedo, G. M. The production of transuranium elements by the r-process nucleosynthesis. Nucl. Phys. A 944, 158 - 176 (2015). URL http://www.sciencedirect.com/science/article/pii/S0375947415001827.

212. Wallner, A. et al. Abundance of live $244 \mathrm{pu}$ in deep-sea reservoirs on earth points to rarity of actinide nucleosynthesis. Nature Communications 6, 1-9 (2015).

213. Perlman, I. \& Seaborg, G. T. The synthetic elements. Scientific American 182, 38-47 (1950).

214. Thompson, S. G., Ghiorso, A. \& Seaborg, G. T. The new element berkelium (atomic number 97). Phys. Rev. 80, 781-789 (1950). URL https://link.aps.org/doi/10.1103/PhysRev.80.781.

215. Seaborg, G. T. \& Bloom, J. L. The synthetic elements: IV. Scientific American 220, 56-69 (1969). URL http://www.jstor .org/stable/24926334.

216. Bohr, N. \& Wheeler, J. A. The mechanism of nuclear fission. Phys. Rev. 56, 426-450 (1939). URL https://link.aps.org/doi/10.1103/PhysRev.56.426. 
217. Reed, B. C. Simple derivation of the Bohr-Wheeler spontaneous fission limit. American Journal of Physics 71, 258-260 (2003). URL https://doi.org/10.1119/1.1526133. https://doi.org/10.1119/1.1526133.

218. Möller, Peter. The limits of the nuclear chart set by fission and alpha decay. EPJ Web Conf. 131, 03002 (2016). URL https://doi.org/10.1051/epjconf/201613103002.

219. Block, M. et al. Direct mass measurements above uranium bridge the gap to the island of stability. Nature 463, 785-788 (2010). URL http://dx.doi.org/10.1038/nature08774.

220. Ramirez, E. M. et al. Direct mapping of nuclear shell effects in the heaviest elements. Science 337, 1207-1210 (2012). URL https://science.sciencemag.org/content/337/6099/1207. https://science.sciencemag.org/content/337/6099/1207.full.pdf.

221. Ito, Y. et al. First direct mass measurements of nuclides around $Z=100$ with a multireflection time-of-flight mass spectrograph. Phys. Rev. Lett. 120, 152501 (2018). URL https://link.aps.org/doi/10.1103/PhysRevLett.120.152501.

222. Block, M. Direct mass measurements and ionization potential measurements of the actinides. Radiochim. Acta 107, 821-831 (2019).

223. Fischer, C. F. Average-energy-of-configuration Hartree-Fock results for the atoms helium to radon. At. Data Nucl. Data Tabl. 12, 87 - 99 (1973). URL http://www.sciencedirect.com/science/article/pii/0092640X73900144. 
224. Flambaum, V. V. \& Ginges, J. S. M. Radiative potential and calculations of qed radiative corrections to energy levels and electromagnetic amplitudes in many-electron atoms. Phys. Rev. A 72, 052115 (2005). URL https://link.aps.org/doi/10.1103/PhysRevA.72.052115.

225. Shabaev, V., Tupitsyn, I. \& Yerokhin, V. Qedmod: Fortran program for calculating the model lamb-shift operator. Comput. Phys. Commun. 189, $175-181$ (2015). URL http://www.sciencedirect.com/science/article/pii/S0010465514004081.

226. Lindgren, I. Relativistic many-body theory: a new field-theoretical approach, vol. 63 (Springer, 2016).

227. Grochala, W., Hoffmann, R., Feng, J. \& Ashcroft, N. W. The chemical imagination at work in very tight places. Angew. Chem. Int. Ed. 46, 3620-3642 (2007).

228. Rahm, M., Cammi, R., Ashcroft, N. W. \& Hoffmann, R. Squeezing all elements in the periodic table: Electron configuration and electronegativity of the atoms under compression. J. Am. Chem. Soc. 141, 1025310271 (2019). URL https://doi.org/10.1021/jacs.9b02634. PMID: 31144505, https://doi.org/10.1021/jacs.9b02634.

229. Schwerdtfeger, P. The pseudopotential approximation in electronic structure theory. ChemPhysChem 12, 3143-3155 (2011). URL https://onlinelibrary.wiley.com/doi/abs/10.1002/cphc.201100387. 
230. Myers, W. D. \& Swiatecki, W. Average nuclear proper$\begin{array}{llllllll}\text { ties. } & \text { Annals of Physics 55, } 395 \quad-505 \text { (1969). }\end{array}$ http://www.sciencedirect.com/science/article/pii/0003491669902024.

231. Nazarewicz, W. Challenges in nuclear structure theory. Journal of Physics G: Nuclear and Particle Physics 43, 044002 (2016). URL https://doi .org/10.1088\%2F0954-3899\%2F43\%2F4\%2F044002.

232. Weizsäcker, C. F. v. Zur Theorie der Kernmassen. Zeit. Physik 96, 431-458 (1935). URL https://doi.org/10.1007/BF01337700.

233. Bethe, H. A. \& Bacher, R. F. Nuclear physics a. station$\begin{array}{lllllllll}\text { ary states of nuclei. Rev. Mod. Phys. } & \mathbf{8}, & 82-229 & \text { (1936). } & \text { URL }\end{array}$ https://link.aps.org/doi/10.1103/RevModPhys.8.82.

234. Kaiser, N., Fritsch, S. \& Weise, W. Nuclear mean field from chiral pion-nucleon dynamics. Nucl. Phys. A 700, 343 - 358 (2002). URL http://www.sciencedirect.com/science/article/pii/S0375947401013148.

235. Yamazaki, T., Kuramashi, Y. \& Ukawa, A. Helium nuclei in quenched lattice QCD. Phys. Rev. D 81, 111504 (2010). URL https://link.aps.org/doi/10.1103/PhysRevD.81.111504.

236. Wiebke, J., Pahl, E. \& Schwerdtfeger, P. Melting at high pressure: Can first-principles computational chemistry challenge diamond-anvil cell experiments? Angew. Chem. Int. Ed. 52, 13202-13205 (2013). URL http://dx.doi.org/10.1002/anie. 201308039. 
237. Schwerdtfeger, P., Tonner, R., Moyano, G. E. \& Pahl, E. Towards j/mol accuracy for the cohesive energy of solid argon. Angew. Chem. Int. Ed. 55, 12200-12205 (2016). URL https://onlinelibrary.wiley.com/doi/abs/10.1002/anie.201605875. https://onlinelibrary.wiley.com/doi/pdf/10.1002/anie.201605875.

238. Bartlett, R. J. \& Musial, M. Coupled-cluster theory in quan$\begin{array}{lllllll}\text { tum chemistry. } \quad \text { Rev. Mod. Phys. } & \mathbf{7 9}, \quad 291-352 \quad \text { (2007). } & \text { URL }\end{array}$ https://link.aps.org/doi/10.1103/RevModPhys.79.291.

239. Coester, F. \& Kümmel, H. Short-range correlations in nuclear wave functions. Nucl. Phys. 17, $477-485$ (1960). $\quad$ URL http://www.sciencedirect.com/science/article/pii/0029558260901401.

240. Čižek, J. \& Paldus, J. Correlation problems in atomic and molecular systems iii. rederivation of the coupled-pair many-electron theory using the traditional quantum chemical methodst. Int. J. Quantum Chem. 5, 359-379 (1971). URL https://onlinelibrary.wiley.com/doi/abs/10.1002/qua.560050402. https://onlinelibrary.wiley.com/doi/pdf/10.1002/qua.560050402.

241. Kümmel, H. G. A biography of the coupled cluster method. Int. J. Mod. Phys. $B$ 17, 5311-5325 (2003). URL https://doi.org/10.1142/S0217979203020442. https://doi.org/10.1142/S0217979203020442.

242. Kowalski, K., Dean, D. J., Hjorth-Jensen, M., Papenbrock, T. \& Piecuch, P. Coupled cluster calculations of ground and excited states of nuclei. Phys. Rev. Lett. 92, 132501 
(2004). URL https://link.aps.org/doi/10.1103/PhysRevLett.92.132501.

243. Hagen, G. et al. Coupled-cluster theory for three-body hamiltonians. Phys. Rev. C 76, 034302 (2007). URL https://link.aps.org/doi/10.1103/PhysRevC.76.034302.

244. Van Dyck, R. S., Zafonte, S. L., Van Liew, S., Pinegar, D. B. \& Schwinberg, P. B. Ultraprecise atomic mass measurement of the $\alpha$ particle and ${ }^{4} \mathrm{He}$. Phys. Rev. Lett. 92, 220802 (2004). URL https://link.aps.org/doi/10.1103/PhysRevLett.92.220802.

245. Piecuch, P. \& Bartlett, R. J. Eomxcc: A new coupled-cluster method for electronic excited states. Adv. Quantum Chem. 34, 295 - 380 (1999). URL http://www.sciencedirect.com/science/article/pii/S0065327608605341.

246. Kane, J. V., Pixley, R. E., Schwartz, R. B. \& Schwarzschild, A. Lifetimes of the first excited states of $\mathrm{F}^{17}$ and $\mathrm{O}^{17}$. Phys. Rev. 120, 162-168 (1960). URL https://link.aps.org/doi/10.1103/PhysRev.120.162.

247. Gour, J. R., Piecuch, P., Hjorth-Jensen, M., Włoch, M. \& Dean, D. J. Coupled-cluster calculations for valence systems around ${ }^{16}$ O. Phys. Rev. C 74, 024310 (2006). URL https://link.aps.org/doi/10.1103/PhysRevC.74.024310.

248. Möller, P., Nix, J., Myers, W. \& Swiatecki, W. Nuclear ground-state masses and deformations. At. Data Nucl. Data Tables 59, 185 - 381 (1995). URL http://www.sciencedirect.com/science/article/pii/S0092640X85710029. 
249. Moller, P. \& Nix, J. R. Stability of heavy and superheavy elements. J. Phys. Nucl. Part. Phys. 20, 1681-1747 (1994). URL https://doi.org/10.1088\%2F0954-3899\%2F20\%2F 11\%2F003.

250. Sadhukhan, J., Dobaczewski, J., Nazarewicz, W., Sheikh, J. A. \& Baran, A. Pairinginduced speedup of nuclear spontaneous fission. Phys. Rev. C 90, 061304 (2014). URL https://link.aps.org/doi/10.1103/PhysRevC.90.061304.

251. Bender, M., Heenen, P.-H. \& Reinhard, P.-G. Self-consistent mean-field models for nuclear structure. Rev. Mod. Phys. 75, 121 (2003).

252. Cook, N. D. Models of the atomic nucleus (Springer-Verlag Berlin Heidelberg, 2006).

253. Oganessian, Y. T., Utyonkov, V. K. \& Moody, K. J. Voyage to superheavy island. Scientific American 282, 63-67 (2000). URL http://www.jstor.org/stable/26058565.

254. Myers, W. D. \& Swiatecki, W. J. Nuclear masses and $\begin{array}{lllllllll}\text { deformations. } & \text { Nucl. } & \text { Phys. } & \mathbf{8 1}, & 1 & - & 60 & \text { (1966). }\end{array}$ http://www.sciencedirect.com/science/article/pii/0029558266906390.

255. Ćwiok, S., Heenen, P. H. \& Nazarewicz, W. Shape coexistence and triaxiality in the superheavy nuclei. Nature 433, 705-709 (2005). URL http://dx.doi.org/10.1038/nature03336.

256. Matheson, Z., Giuliani, S. A., Nazarewicz, W., Sadhukhan, J. \& Schunck, N. Cluster radioactivity of ${ }_{118}^{294} \mathrm{Og}_{176}$. Phys. Rev. C 99, 041304 (2019). URL https://link.aps.org/doi/10.1103/PhysRevC.99.041304. 
257. Ćwiok, S., Dobaczewski, J., Heenen, P. H., Magierski, P. \& Nazarewicz, W. Shell structure of the superheavy elements. Nucl. Phys. A 611, 211-246 (1996). URL http://linkinghub.elsevier.com/retrieve/pii/S0375947496003375.

258. Kruppa, A. T. et al. Shell corrections of superheavy nuclei: Green's function Hartree-Fock method. Phys. Rev C 61, 034313 (2000). URL http://link.aps.org/doi/10.1103/PhysRevC.61.034313.

259. Morita, K. et al. Experiment on the synthesis of element 113 in the reaction ${ }^{209} \operatorname{Bi}\left({ }^{70} \mathrm{Zn}, n\right){ }^{278} 113$. J. Phys. Soc. Japan 73, 2593-2596 (2004).

260. Münzenberg, G. \& Morita, K. Synthesis of the heaviest nuclei in cold fusion reactions. Nucl. Phys. A 944, 3 - 4 (2015). URL http://www.sciencedirect.com/science/article/pii/S0375947415001360.

261. Oganessian, Y. T. et al. Synthesis of the isotopes of elements 118 and 116 in the ${ }^{249} \mathrm{Cf}$ and ${ }^{245} \mathrm{Cm}+{ }^{48} \mathrm{Ca}$ fusion reactions. Phys. Rev. C 74, 044602 (2006). URL https://link.aps.org/doi/10.1103/PhysRevC.74.044602.

262. Oganessian, Y. Heaviest nuclei from 48Ca-induced reactions. J. Phys. G: Nucl. Part. Phys. 34, R165-R242 (2007). URL https ://doi .org/10.1088\%2F0954-3899\%2F34\%2F4\%2Fr01.

263. Hofmann, S. Super-heavy nuclei. J. Phys. G 42, 114001 (2015). URL http://stacks . iop. org/0954-3899/42/i=11/a=114001. 
264. Kragh, H. The search for superheavy elements: Historical and philosophical perspectives. arXiv preprint arXiv:1708.04064 (2017).

265. Restrepo, G. Challenges for the Periodic Systems of Elements: Chemical, Historical and Mathematical Perspectives. Chemistry - A European Journal 25, 15430-15440 (2019). URL https://onlinelibrary.wiley.com/doi/abs/10.1002/chem.201902802. https://onlinelibrary.wiley.com/doi/pdf/10.1002/chem.201902802.

266. Scerri, E. Can quantum ideas explain chemistry's greatest icon? Nature 565, 557-559 (2019).

267. Scerri, E. \& Restrepo, G. Mendeleev to Oganesson: a multidisciplinary perspective on the periodic table (Oxford University Press, 2018).

268. Scerri, E. Cracks in the periodic table. Scientific American 308, 68-73 (2013).

269. Scerri, E. R. The periodic table. In Woody, A. I., Hendry, R. F. \& Needham, P. (eds.) Philosophy of Chemistry, vol. 6 of Handbook of the Philosophy of Science, 329 - 338 (North-Holland, Amsterdam, 2012). URL http://www.sciencedirect.com/science/article/pii/B9780444516756500244.

270. Kutzelnigg, W. The periodic table. its story and its significance. Int. J. Quantum Chem. 110, 1443-1444 (2010). URL https://onlinelibrary.wiley.com/doi/abs/10.1002/qua. 22276. https://onlinelibrary.wiley.com/doi/pdf/10.1002/qua. 22276. 
271. Schädel, M. Chemistry of the superheavy elements. Phil. Trans. Royal Soc. Lond. A 373, 20140191 (2015).

272. Kirsebom, O. S. et al. Discovery of an exceptionally strong $\beta$-decay transition of ${ }^{20} \mathrm{~F}$ and implications for the fate of intermediate-mass stars. Phys. Rev. Lett. 123, 262701 (2019). URL https://link.aps.org/doi/10.1103/PhysRevLett.123.262701.

273. Meyer, L. Die Natur der chemischen Elemente als Funktion ihrer Atomgewichte. $A n$ nalen Chem. Pharm.: Supplementband 7, 354-364 (1870).

274. van Spronsen, J. W. The priority conflict between mendeleev and meyer. J. Chem. Ed. 46, 136 (1969).

275. Kuhn, N. \& Zeller, K.-P. Lothar meyer - eine wiederentdeckung. Nachrichten aus der Chemie 67, 19-25 (2019). URL https://onlinelibrary.wiley.com/doi/abs/10.1002/nadc. 20194090181. https://onlinelibrary.wiley.com/doi/pdf/10.1002/nadc. 20194090181.

276. Boeck, G. Das Periodensystem der Elemente und Lothar Meyer. Chemie in unserer Zeit $\mathbf{0}$ (2019). URL https://onlinelibrary.wiley.com/doi/abs/10.1002/ciuz. 201900001. https://onlinelibrary.wiley.com/doi/pdf/10.1002/ciuz. 201900001.

277. Schwerdtfeger, P. Relativistic and electron-correlation contributions in atomic and molecular properties: benchmark calculations on 
$\mathrm{Au}$ and $\mathrm{Au}_{2}$. Chem. Phys. Lett. 183, 457 - 463 (1991). URL http://www.sciencedirect.com/science/article/pii/0009261491904093.

278. Schwerdtfeger, P. Relativistic effects in properties of gold. Heteroat. Chem. 13, 578-584 (2002). URL http://dx.doi.org/10.1002/hc.10093.

279. Pyykkö, P. Theoretical chemistry of gold. Angew. Chem. Int. Ed. 43, 4412-4456 (2004). URL http://dx.doi.org/10.1002/anie.200300624.

280. Glantschnig, K. \& Ambrosch-Draxl, C. Relativistic effects on the linear optical properties of $\mathrm{u}, \mathrm{Pt}, \mathrm{Pb}$ and W. New J. Phys. 12, 103048 (2010). URL https://doi.org/10.1088\%2F $1367-2630 \% 2 \mathrm{~F} 12 \% 2 \mathrm{~F} 10 \% 2 \mathrm{~F} 103048$.

281. Schwerdtfeger, P. Gold goes nano - from small clusters to low-dimensional assemblies. Angew. Chem. Int. Ed. 42, 1892-1895 (2003). URL https://onlinelibrary.wiley.com/doi/abs/10.1002/anie.200201610. https://onlinelibrary.wiley.com/doi/pdf/10.1002/anie. 200201610.

282. Theilacker, K., Schlegel, H. B., Kaupp, M. \& Schwerdtfeger, P. Relativistic and solvation effects on the stability of gold(III) halides in aqueous solution. Inorg. Chem. 54, 9869-9875 (2015). URL https ://doi .org/10.1021/acs . inorgchem.5b01632. PMID: 26421633, https://doi.org/10.1021/acs. inorgchem.5b01632.

283. Hashmi, A. S. K. The catalysis gold rush: New claims. Angew. Chem. Int. Ed. 44, 6990-6993 (2005). URL 
https://onlinelibrary.wiley.com/doi/abs/10.1002/anie. 200502735.

https://onlinelibrary.wiley.com/doi/pdf/10.1002/anie. 200502735.

284. Gorin, D. J. \& Toste, F. D. Relativistic effects in homogeneous gold catalysis. Nature 446, 395 (2007).

285. St. Andrews' find may be oldest surviving wall chart of periodic table, The Guardian, January 17, see: https://www.theguardian.com/science/2019/jan/17/standrews-mldest-surviving-wall-chart-of-periodic-table-university.

286. Seaborg, G. T. The periodic table tortuous path to man-made elements. Chemical and Engineering News 57, 46-52 (1979).

287. Haba, H. A new period in superheavy-element hunting. Nature Chem. 11, 10-13 (2019). Doi.org/10.1038/s41557-0180191-8.

288. Johnson, J. A. Populating the periodic table: Nucleosynthesis of the elements. Science $\mathbf{3 6 3}, \quad 474-478 \quad$ (2019). URL https://science.sciencemag.org/content/363/6426/474. https://science.sciencemag.org/content/363/6426/474.full.pdf.

289. Aker, M. et al. An improved upper limit on the neutrino mass from a direct kinematic method by katrin. arXiv preprint arXiv:1909.06048 (2019).

290. Lackenby, B. G. C., Dzuba, V. A. \& Flambaum, V. V. Theoretical calculation of atomic properties of superheavy elements $Z=110-112$ and their ions. arXiv preprint

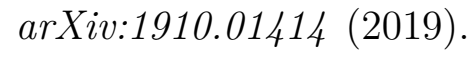


291. Schwerdtfeger, P. \& Seth, M. Relativistic effects of the superheavy elements. In Schleyer, P. v. R. et al. (eds.) Encyclopedia of Computational Chemistry, vol. 4, 2480-2499 (John Wiley \& Sons, Ltd, 1998). URL http://dx.doi.org/10.1002/0470845015.cra007.

292. Kramida, A., Yu. Ralchenko, Reader, J. \& and NIST ASD Team. NIST Atomic Spectra Database (ver. 5.6.1), [Online]. Available: https://physics.nist.gov/asd [2019, September 26]. National Institute of Standards and Technology, Gaithersburg, MD. (2018).

293. Eliav, E., Kaldor, U., Ishikawa, Y., Seth, M. \& Pyykkö, P. Calculated energy levels of thallium and eka-thallium (element 113). Phys. Rev. A 53, 3926-3933 (1996). URL https://link.aps.org/doi/10.1103/PhysRevA.53.3926.

294. Eliav, E. \& Kaldor, U. Four-component electronic structure methods. In Relativistic methods for chemists, 279-349 (Springer, 2010).

295. Rolfs, C. E., Rodney, W. S. \& Rodney, W. S. Cauldrons in the cosmos: Nuclear astrophysics (University of Chicago press, Chicago, 1988).

296. Zagrebaev, V. I. \& Greiner, W. Cross sections for the production of superheavy nuclei. Nucl. Phys. A 944, $257-307$ (2015). URL http://www.sciencedirect.com/science/article/pii/S037594741500041X.

297. Oganessian, Y. T. Synthesis of the heaviest elements in 48Ca-induced reactions. $R a-$ diochim. Acta 99, 429-439 (2011). 
298. Roederer, I. U. et al. New detections of Arsenic, Selenium, and other heavy elements in two metal-poor stars. Astrophys. J. 791, 32 (2014). URL https://doi.org/10.1088\%2F0004-637x\%2F791\%2F1\%2F32.

\section{Box 1. A bit of history: Dmitri Ivanovich Mendeleev and Lothar Meyer}

The International Year of the Periodic Table (IYPT) in 2019 commemorated the 1869 papers of Dmitri Ivanovich Mendeleev (picture top right). Five years earlier, Lothar Meyer (picture bottom right) had introduced in his 1864 book Die Modernen Theorien der Chemie (1st ed., p.137), a 28-element table with six columns labelled by valence, and five rows with increasing atomic weight, correcting the $\mathrm{Te} / \mathrm{I}$ anomaly. Meyer's columns correspond to the Groups 1-2 and 14-17. He did not claim new elements, did not have groups 3-13, nor explicitly mention periodicity. He also attributed valencies to certain transition metals from modern groups 4-12. Meyer later commented (translated from German): "Recently, Mendeleyeff has shown that such an arrangement can already be obtained by simply arranging atomic weights of all elements without random selection into a single row according to the size of their numerical values, decomposing such a row into sections and putting them together in the unmodified sequence. The table shown below is essentially identical to that given by Mendeleyeff". ${ }^{273-275}$ We refer to an excellent historical discourse into Lothar Meyer's life and work and comments on this issue by Gisela Boeck. ${ }^{276}$ (Both pictures are wikipedia with no license attached.) 


\section{Box 2. The many-electron Dirac-Fock-Breit Hamiltonian}

The many-electron Dirac-Fock-Breit Hamiltonian can be considered a chemist's 'Theory of Everything'.

The many-electron Hamiltonian can be expressed as the sum of one-electron $\left(h_{i}\right)$ and two-electron $\left(h_{i j}\right)$ Hamiltonians:

$$
H=\sum_{i} h_{i}+\sum_{i<j} h_{i j}
$$

The non-relativistic one-particle Hamiltonian would be (in atomic units):

$$
h=T+V_{n}, \quad T=-\frac{1}{2} \nabla^{2}
$$

with $T$ being the kinetic energy, $V_{n}$ the potential energy and $\nabla^{2}$ the Laplace operator. And the non-relativistic two-particle Hamiltonian would be:

$$
h_{i j}=1 / r_{i j}
$$

where, $r_{i j}$ is the distance between the two particles.

The one-particle Dirac Hamiltonian $\left(h_{\mathrm{D}}\right)$ takes into account relativistic effects and it reads as:

$$
h_{\mathrm{D}}=c \vec{\alpha} \cdot \vec{p}+\beta c^{2}+V_{n}, \quad \vec{p}=-i \vec{\nabla}
$$

where $\alpha_{i}$ and $\beta$ are Dirac matrices, $\vec{p}$ is the momentum and $c=137.035999$ au is the speed of light. The relativistic two-particle Hamiltonian $\left(h_{\mathrm{B}}\right)$ can be obtained by including in Eq. 3 the Breit interaction (here chosen as frequency-independent) that describes the magnetic electron-electron interactions and retardation effects.

$$
h_{\mathrm{B}}=-\frac{1}{2 r_{i j}}\left[\vec{\alpha}_{i} \cdot \vec{\alpha}_{j}+\left(\vec{\alpha}_{i} \cdot \vec{r}_{i j}\right)\left(\vec{\alpha}_{j} \cdot \vec{r}_{i j}\right) / r_{i j}^{2}\right]
$$

In correlated calculations, electron-like projection operators, $P$, should be added:

$$
h_{i j}^{e f f}=P h_{i j} P \text {. }
$$

Quantum electrodynamics (QED) corrections can also be added if we want to achieve higher accuracy. For more details see Ref.30. 


\section{Box 3. A head-on comparison of theory and experiment for the Au atom}

The ionization potential (IP) and electron affinity (EA) of the Au atom have been accurately measured by laser experiments. Pašteka et al. ${ }^{69}$ reached theoretical millielectronvolt accuracy by performing highly correlated calculations with a coupledcluster method having up to pentuple excitations (DC-CCSDTQP) in a large basis $\underline{\text { (values are in } \mathrm{eV}) .} .^{69}$

\begin{tabular}{lcccc}
\hline Method & \multicolumn{2}{c}{ IP } & \multicolumn{2}{c}{ EA } \\
\hline DC-HF & Value & Error & Value & Error \\
DC-CCSDTQP & 9.6892 & 1.5364 & 0.6690 & 1.6396 \\
+ Breit & 9.2701 & 0.0446 & 2.3278 & 0.0192 \\
+ QED & 9.22846 & 0.0290 & 2.3188 & 0.0102 \\
Exp. & 9.2256 & 0.0032 & 2.3072 & -0.0014 \\
\hline
\end{tabular}




\section{Box 4. Au is inherently relativistic.}

High-level calculations for the Au ionization potential $E_{\mathrm{IP}}$ and electron affinity $E_{\mathrm{EA}}{ }^{69}$ show that electron correlation contributes $1.565 \mathrm{eV}$ (17.0\% of the experimental value) to $E_{\mathrm{IP}}$ and $1.650 \mathrm{eV}(71.5 \%)$ to $E_{\mathrm{EA}}$ (at the Dirac-Coulomb-Breit level of theory). The same calculations show that relativistic effects contribute $2.140 \mathrm{eV}(23.2 \%)$ to $E_{\mathrm{IP}}$ and $1.012 \mathrm{eV}(43.8 \%)$ to $E_{\mathrm{EA}}$ (at the coupled cluster level of theory). According to Mulliken's definition of the electronegativity, $\chi$ of gold, the value $\chi=0.187\left(E_{\mathrm{IP}}+E_{\mathrm{EA}}\right)$ $\left(E_{\mathrm{IP}}\right.$ and $E_{\mathrm{EA}}$ taken in units of $\mathrm{eV}$ ) translates into a relativistic change ${ }^{277}$ of $\Delta_{R} \chi=$ 0.59. As a result, $\chi_{\mathrm{Au}}$ is substantially increased, by $\sim 2.4$, and Au can be regarded both as a metal and as a pseudo-halide. Therefore, relativistic effects influence the whole chemistry and physics of Au leading to well known anomalies in properties compared to those of $\mathrm{Cu}$ and $\mathrm{Ag}$, as shown in the Table below ${ }^{79,278,279}$. The most striking features to be highlighted are the yellow colour of bulk $\mathrm{Au}^{280}$, the catalytic activity and unusual structures of $\mathrm{Au}$ nanoclusters ${ }^{281}$ and the high stability of high oxidation states, such as for the Au halides ${ }^{282}$, and its associated role in homogeneous catalysis $^{283,284}$.

\begin{tabular}{l|lll} 
Property & Copper & Silver & Gold \\
\hline Colour & bronze & silver & yellow \\
Specific resistivity $\left(10^{-8} \Omega \mathrm{m}\right)$ & 1.72 & 1.62 & 2.4 \\
Thermal conductivity $\left(\mathrm{W} \mathrm{cm}{ }^{-1} \mathrm{~K}^{-1}\right)$ & 3.85 & 4.18 & 3.1 \\
Electronic heat capacity $\left(10^{-4} \mathrm{~J} \mathrm{~K}^{-1} \mathrm{~mol}^{-1}\right)$ & 6.926 & 6.411 & 6.918 \\
Melting point $\left({ }^{\circ} \mathrm{C}\right)$ & 1083 & 961 & 1064 \\
Boiling point $\left({ }^{\circ} \mathrm{C}\right)$ & 2567 & 2212 & 3080 \\
Atomic volume $\left(\mathrm{cm}^{3} \mathrm{~mol}^{-1}\right)$ & 7.12 & 10.28 & 10.21 \\
Electronegativity & 1.9 & 1.9 & 2.4 \\
Polarizability $(\mathrm{au})$ & 46.5 & 55 & 36 \\
Cohesive energy $(\mathrm{kJ}$ mol & \\
Desorption temperature CO on metal surface $(\mathrm{K})$ & $190-210$ & $40-80$ & $170-180$ \\
Common oxidation states & I,II & I & I,III \\
\hline
\end{tabular}


Box 5. The astrophysical $s$ - and $r$-neutron capture process

Neutron capture and $\beta$ decay produce the heaviest elements in the periodic table. During the rapid neutron capture process ( $r$-process), which occurs in supernovae and neutron star mergers, there are many possible reaction paths and the unstable nucleus captures another neutron before decaying. The $r$-process operates under conditions far from stability, closer to the neutron drip line. During the slow neutron capture process ( $s$-process), which occurs in stars (particularly asymptotic giant branch (AGB) stars), the unstable nucleus decays before capturing another neutron and the path is close to the valley of $\beta$-stability, as shown in the figure with half-lives included (Data from Ref.164). 


\section{Box 6. Nuclear structure calculations}

Nuclear structure theory is far more complicated than electronic structure theory owing to the strong and electromagnetic interactions of the neutrons and protons. The nuclear interaction is quite complex, involving manybody forcess. However, nuclear structure theory has developed rapidly over the past few decades due to improved theoretical methods and parametrizations, algorithms and computer power. In a very similar fashion to quantum chemistry, nuclear structure theory ranges from more semi-empirical to more accurate methods (alternatively wavefunction or density functional based). An overview over the different levels of approximations in use is given on the right (adopted from Ref. 231; (C)IOPscience, Journal of Physics G).

Figure 1: Periodic Tables. (a) Earliest example (1885) of a wall-hanging PTE (purchased in 1888 and recently restored by the University of St. Andrews.) Figure adapted with permission from Ref. 285. (b) A 1942 PTE by Glenn T. Seaborg (Figure adapted with permission from the internal Lawrence Berkeley Laboratory document with reference number XBL 798-2509, Ref.286). In this version the $5 f$ elements have not been introduced yet; Th is shown in the group below Hf, Pa below Ta, and U below W. The actinide series got its proper place in the PTE in 1944. (c) PTE assigning a single electron configuration to each atom. ${ }^{10}$ The yellow cases are now experimentally known. Note the suggested location of a putative $5 \mathrm{~g}$ series. The number of valence electrons is given by the Group number, as $G$ (Groups 1-12), or $G-10$ (Groups 13-18). The length and the location of the rows reflect both the chemistry of the elements and the shell-structure of their atoms. Figure adapted with permission from Ref. 287, NPG. (d) PTE showing the predicted origin of elements in the Solar System. Elements beyond plutonium are not included. Figure adapted with permission from Ref.288, AAAS. 
Figure 2: The Standard Model of Fundamental Particles ("The physicist's periodic table"). The electron neutrino mass with the new upper limit at $1.1 \mathrm{eV}$ is used. ${ }^{289}$ Blue lines indicate with which fermions the gauge bosons interact. (Note that in this review we use energy units of $\mathrm{eV}$ for spectroscopic and nuclear properties, i.e. $1 \mathrm{eV}=96.4853075 \mathrm{~kJ} / \mathrm{mol}$. Masses are given in energy units through $E=m c^{2}$ and $1 \mathrm{amu}=931.49410242 \mathrm{MeV}$. For bond energies we use $\mathrm{kJ} / \mathrm{mol}$.)

Figure 3: Electronic States and Configurations. (a) NIST energy levels for the dominant configurations of the Group 10 elements. Different colors are used to distinguish between the three different configurations: green $\left[(n-1) d^{8} n s^{2}\right]$, orange $\left[(n-1) d^{9} n s^{1}\right]$ and maroon $\left[3 d^{10}\right]$. Note that for Pd we already have intruder states (not shown here) arising from the $[(n-$ 1) $\left.d^{9} n p^{1}\right]$ configuration (for Pt from the $\left[(n-1) d^{9} n p^{1}\right]$ and $\left[(n-1) d^{8} n s^{1} p^{1}\right]$ configurations), which mix with several of the low energy states shown here. Thus, some configuration assignments (especially for the ${ }^{3} P_{0}$ level) are approximate at best. For Ds a dense spectrum arising from the $\left[6 d_{5 / 2}^{7} 7 s^{2} 7 p_{1 / 2}^{1}\right]$ configuration intrudes into the normal spectrum and only few predicted lines of even parity by Lackenby et al. are given here. Data adapted with permission from Ref.290. (b) Successive shell filling according to Fig.1c, up to the heaviest element with $Z=172$ according to the Madelung-Janet rule. We give here the anomalies in expected shell filling, highlighted in blue. They result from strong relativistic stabilization effects of the $s$ and $p_{1 / 2}$ shells at high nuclear charge. Figure reproduced with permission from Ref. 131, EDP. (c) Orbital energies and (d) configuration energies for the $3 d, 4 d$ and $5 d$ transition metal atoms (in eV). Data was adapted with permission from Ref. 64, ACS, labelled as "theoretical" in their paper. 
Figure 4: Relativistic Effects. (a) Ratio of the relativistic (R) to non-relativistic (NR) expectation values of average orbital radii, $\langle r\rangle$, for different Dirac-Hartree-Fock $(n l j)$-orbitals in $\mathrm{Og}(Z=118)$. (b) NR and $\mathrm{R}$ radial densities for the valence $7 \mathrm{~s}$ and $7 \mathrm{p}$ orbitals of $\mathrm{Og}$. Corresponding orbital energies are given in the right upper panel of the figure. Figure adapted with permission from Ref. 109, ACS. (c) Relativistic stabilization of the $n s$ shell for the elements $\mathrm{K}$ to $\mathrm{Kr}(n=4)$, Rb to Xe $(n=5)$, Cs to $\mathrm{Rn}(n=6)$, and Fr to $\mathrm{Og}(n=7)$. Redrawn from the data and configurations given by Desclaux ${ }^{78}$ and from numerical HartreeFock calculations (for $\mathrm{Pd}$ the $4 d^{9} 5 s^{1}$ and for Tc the $4 d^{5} 5 s^{2}$ configurations were chosen). See Ref. 71, 291 for details. (d) Spin-orbit splitting (in eV) for the group $13\left({ }^{2} \mathrm{P}\right)$, group $14\left({ }^{3} \mathrm{P}\right)$, group $16\left({ }^{3} \mathrm{P}\right)$ and group $17\left({ }^{2} \mathrm{P}\right)$ elements of the PTE. Data taken from National institute of standards and technology (NIST), ${ }^{292}$ for At and the superheavy elements from Refs. 293, 294 and from Dirac-Hartree-Fock-Breit+QED calculations. (e) R and NR free reaction energies (in $\mathrm{kJ} / \mathrm{mol}$ ) for the decomposition $\mathrm{ECl}_{4} \rightarrow \mathrm{Cl}_{2}+\mathrm{ECl}_{2}(\mathrm{E}=\mathrm{C}-\mathrm{Pb}$ ) at the density functional level of theory. Data obtained from Refs.19,116. (f) Diving of the $1 s$ and $2 s$ hydrogenic states into the negative-energy continuum for different levels of theory: NR Schrödinger equation with a point nuclear charge (PNC); Dirac-Coulomb equation (Dirac) with a point nuclear charge ending abruptly at $Z_{\text {crit }}^{\mathrm{PNC}}$; Dirac-Coulomb equation with a finite nuclear charge distribution (FNC) using a two-parameter Fermi model. ${ }^{80}$ Energies are in units of $m_{e} c^{2}$ and onset of positive energy continuum is set to zero. 
Figure 5: Localization functions. (a) Electron localization functions from non-relativistic (NR, left) and Dirac-Hartree- Fock calculations ( $\mathrm{R}$, right) for the heavy rare gas atoms Xe (top), Rn (middle), and Og (bottom). Adapted with permission from Ref. 77, APS. (b) Nucleon localization function for a highly deformed configuration of ${ }_{118}^{294} \mathrm{Og}$ for neutrons and protons. For comparison, localizations are shown for the prefragments ${ }_{82}^{208} \mathrm{~Pb}$ and ${ }_{36}^{86} \mathrm{Kr}$ on the left side of each subplot. Adapted with permission from Ref.256, APS. 
Figure 6: Nuclear Structure. (a) Chart of known and predicted (grey zone) nuclei colored by their half-lives. The grey zone ends at the proton and neutron drip lines. Original graph with permission from Brad Sherrill using half-lives extracted from National Nuclear Data Center, NuDat2 database, www.nndc.bnl.gov. The $r$-process (near the neutron drip line) and the $s$-process (near the region of stability) trajectories shown here are one of many possible pathways (data adopted from Ref. 295). (b) Chart of nuclides (isotopes) by binding energy per nucleon depicting the valley of stability. Data obtained from the NuDat2 database of the National Nuclear Data Center (www.nndc.bnl.gov). (c) Adiabatic potential energy of the ${ }_{20}^{48} \mathrm{Ca}+{ }_{96}^{248} \mathrm{Cm}$ collision from a two-center shell model. Different reaction channels (deep inelastic scattering, quasi-fission and fusion) are shown schematically by white arrows.) Picture adapted with permission from Ref. 296, Elsevier. (d) Predicted ground-state mass quadrupole deformation $\beta_{2}$ in the $(N, Z)$-plane for even-even superheavy nuclei obtained from DFT calculations with a SLy4 nuclear energy density functional. The centre of the shell stability is predicted around $N=184, Z=126$. Prolate shapes $\left(\beta_{2}>0\right)$ are coloured redorange, oblate shapes $\left(\beta_{2}<0\right)$ blue-green, and spherical shapes $\left(\beta_{2}=0\right)$ light yellow. Picture adapted with permission from Ref.255, NR. (e) Areas of stability and two examples of cold and hot fusion reactions and their $\alpha$-decay products in the superheavy element (SHE) region. The red center in the SHE region around the magic number $Z=114$ and $N=184$ indicates enhanced shell stability. With courtesy of Yuri Oganessian; greyscale version of published in Ref. 297. (f) Timeline of discovery of the actinide and trans-actinide elements including the original discovery of uranium by Klaproth in 1789 . 
Figure 7: Abundancies of Elements. (a) Heavy-element abundance patterns in the metalpoor star HD108317 shown in the right upper corner (constellation Virgo at 720 light years distance). Black squares indicate detections from the Space Telescope Imaging Spectrograph on board the Hubble Space Telescope, and open downward-pointing triangles indicate upper limits from non-detections. The solid red lines represent the $r$-process pattern, normalized to $\mathrm{Eu}$, and the blue lines represent the solar system $s$-process pattern, normalized to Ba. The bottom panels show the abundance residuals with respect to the $r$-process pattern. Figure adapted with permission from Ref.298, IOP. (b) Simulation under cold $r$-process conditions utilizing the Thomas-Fermi Model with Strutinski Integral method for fission barrier and mass predictions. The color coded abundances (right had side of the plot) are given at the point of neutron freeze-out, i.e. when the ratio of neutrons to heavy nuclei has dropped down to 1 after $t=0.7876$ s. Figure adapted with permission from Ref.210, EDP. 
Figure 1

a

Periodische Gesetzmässigkeit der Elemente nach Mendelejeff.

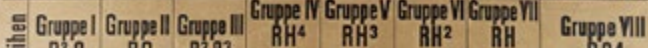

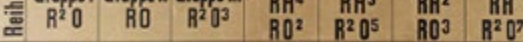
$2 \mathrm{Li}_{2}=7_{\mathrm{H}=\mathrm{I}}^{\mathrm{Be}=9.4 \text { B. }}$

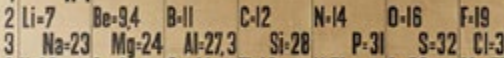

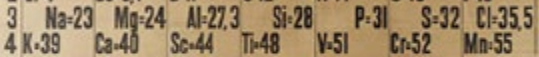

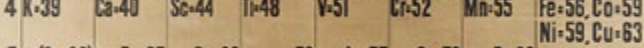

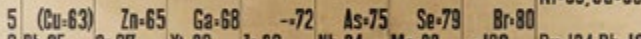

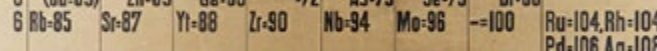

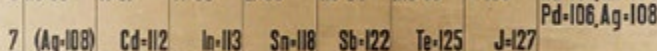
8 Csil33 Ba.437 Ce. 137 la.139

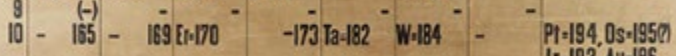

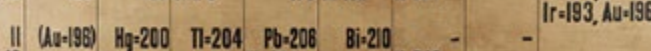

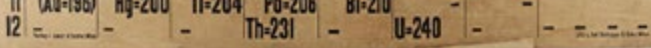

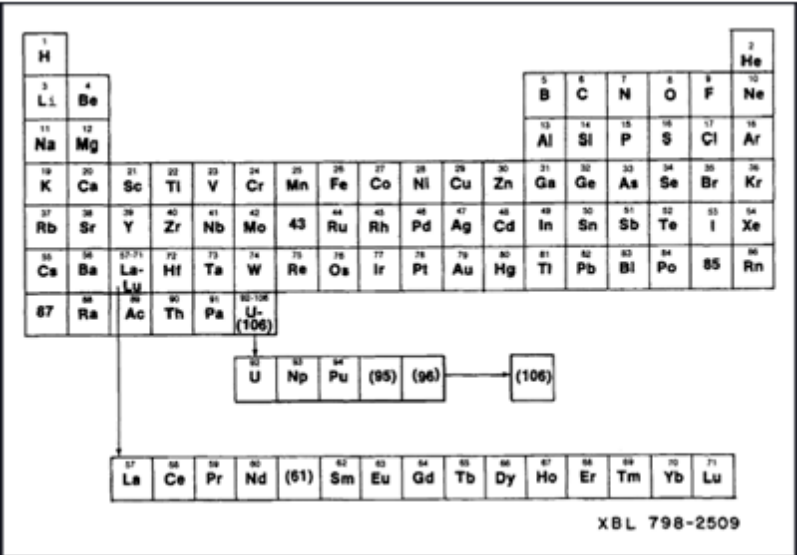

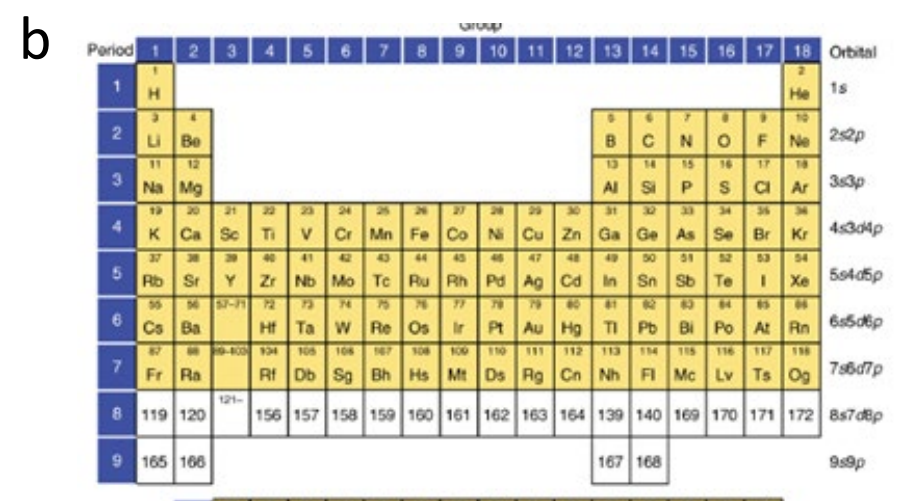

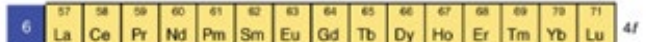

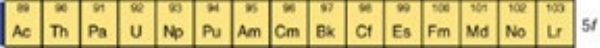

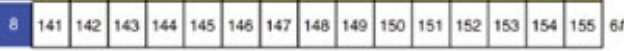

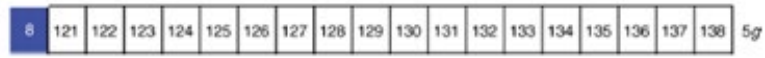


Figure 2

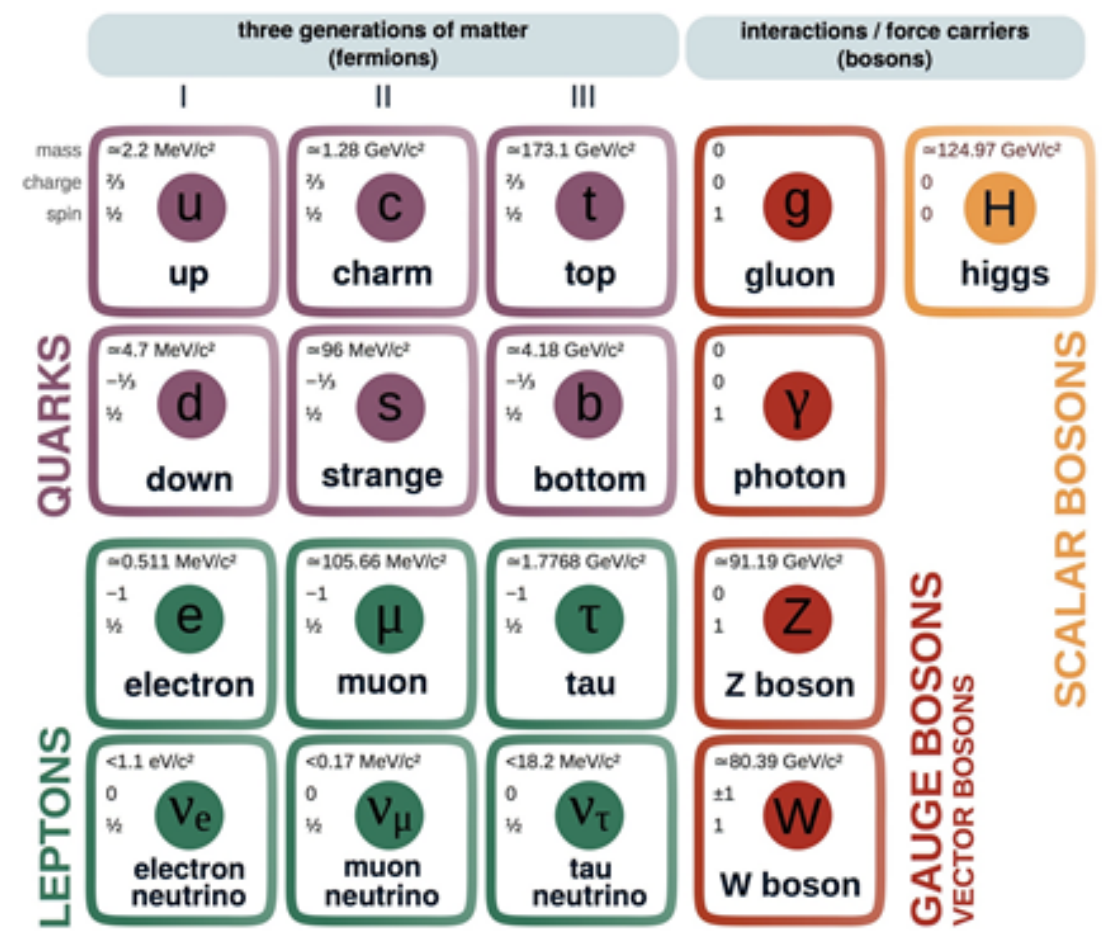




\section{Figure 3}

(a)

(c)
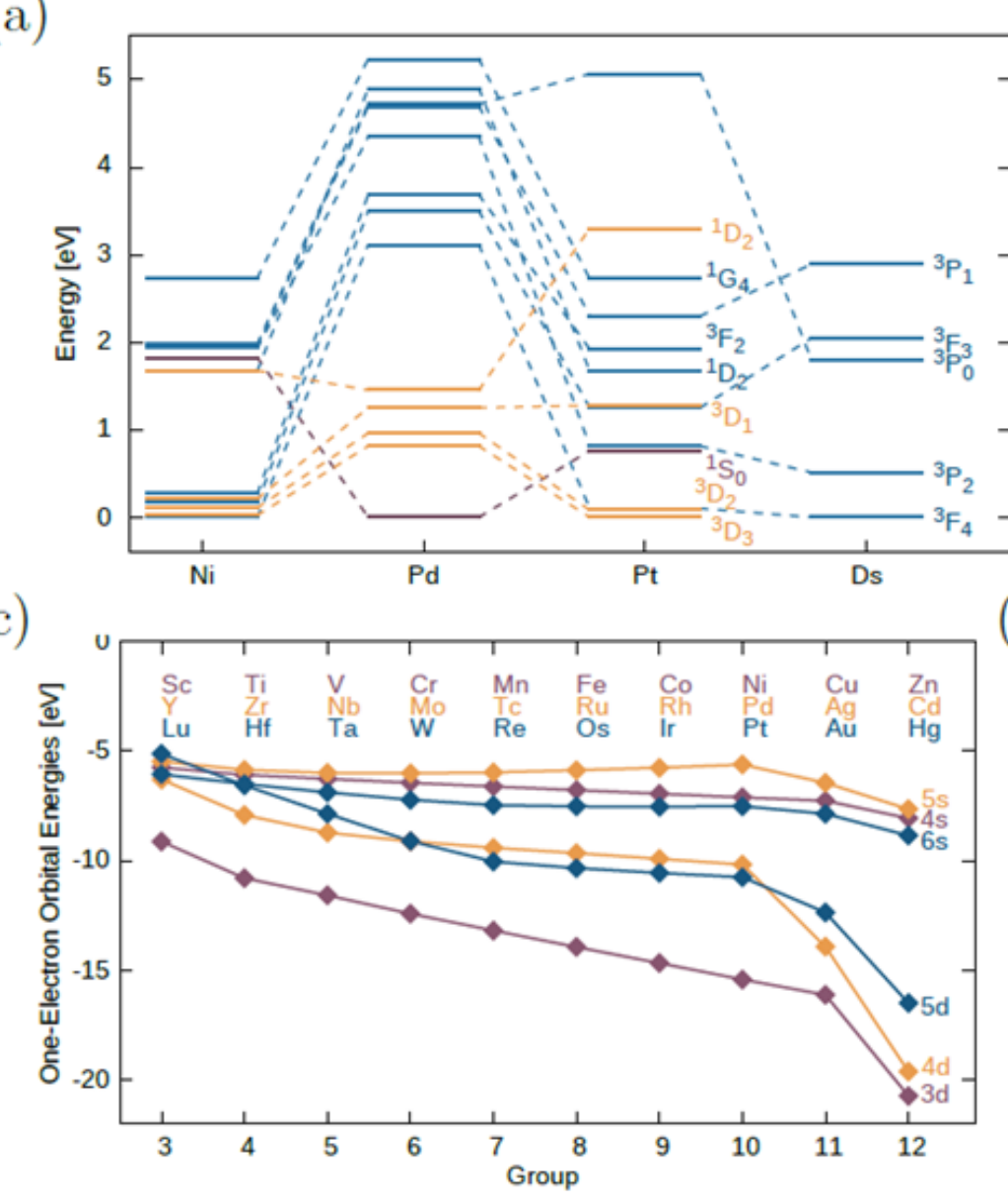

(b)

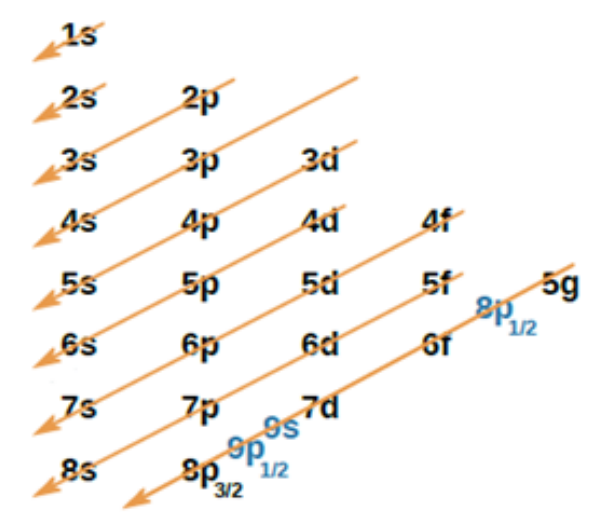

(d)

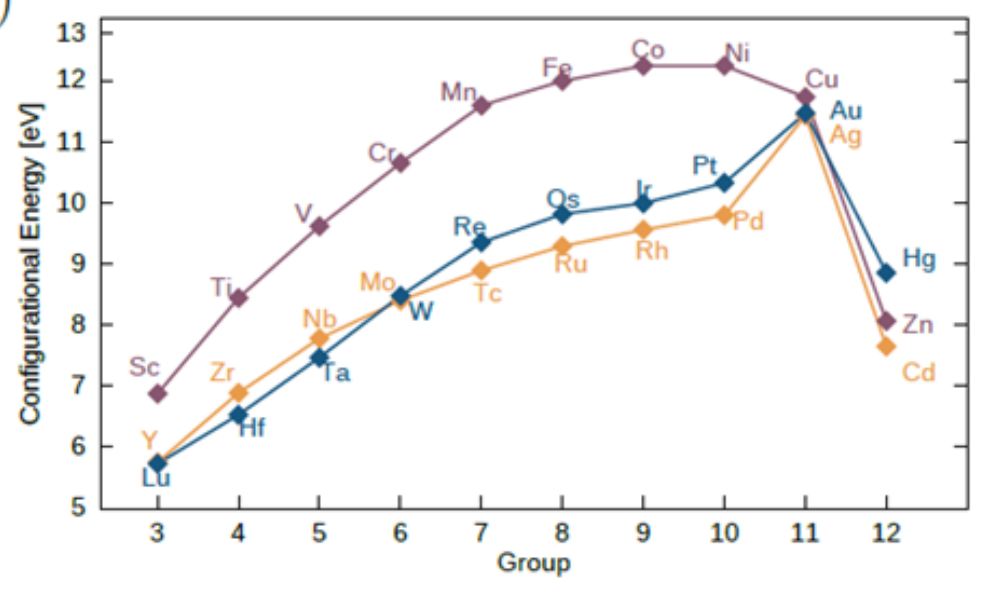


Figure 4

(c)
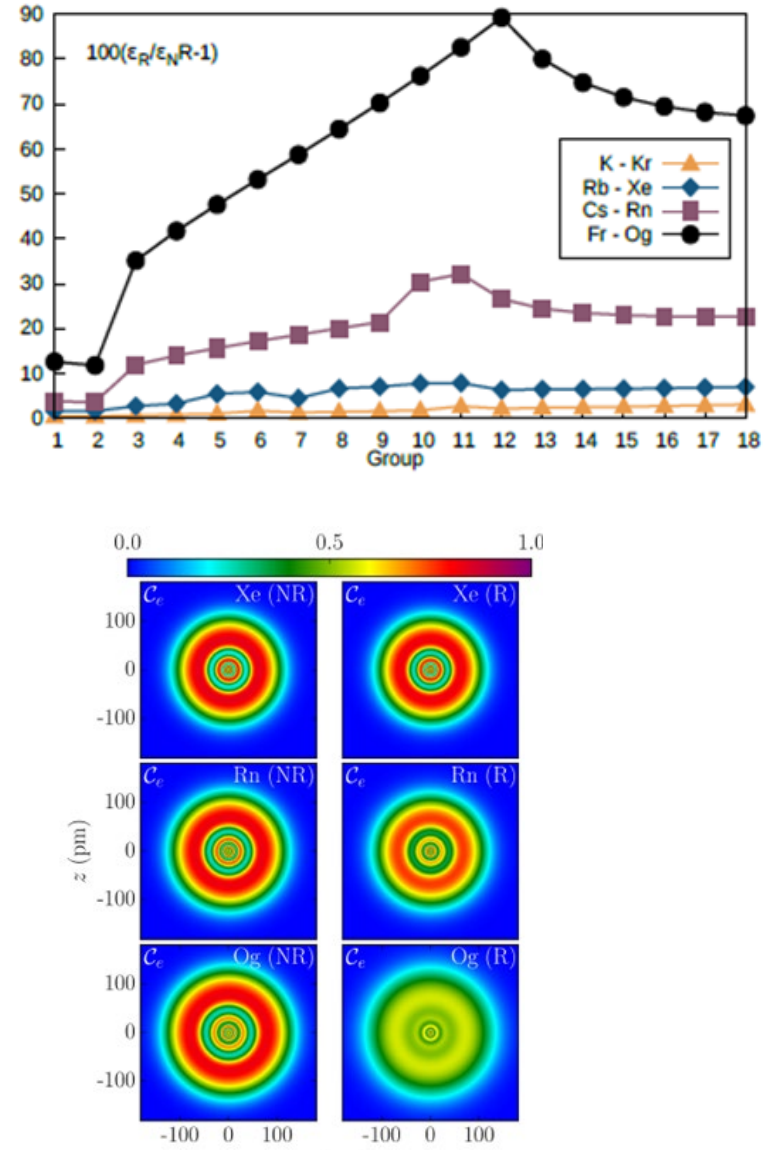

(e)

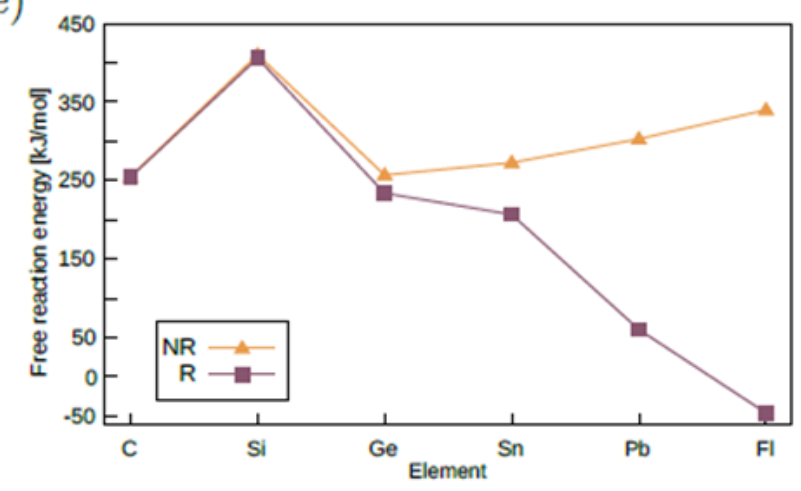

(d)

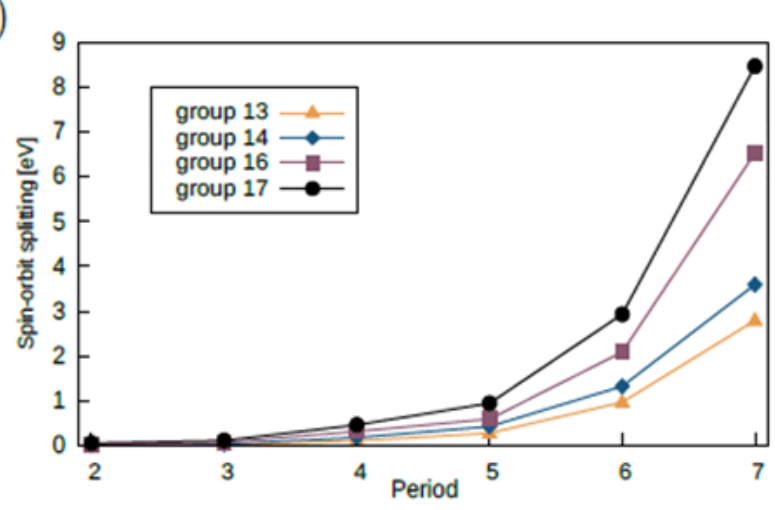

(b)

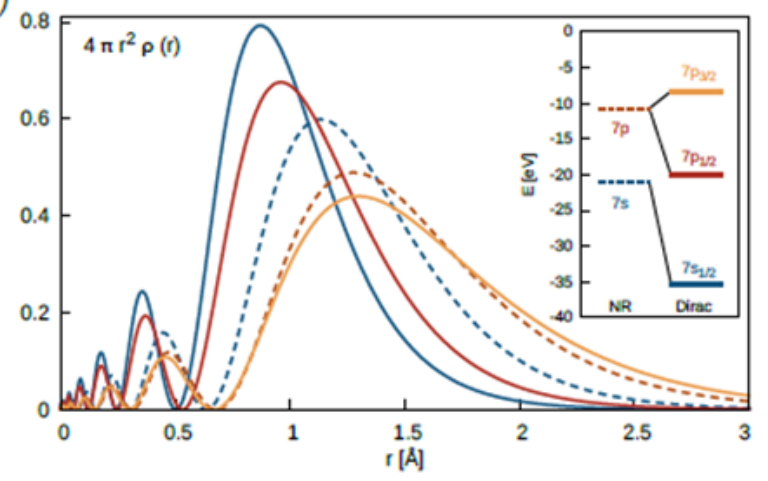

(f)

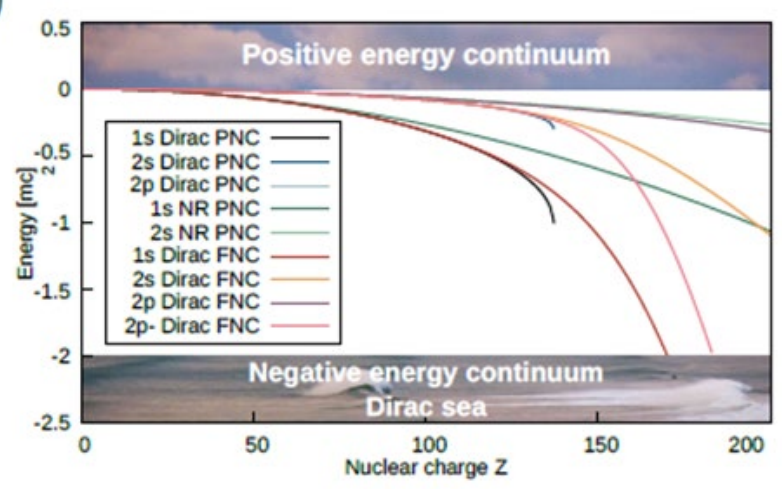


Figure 5

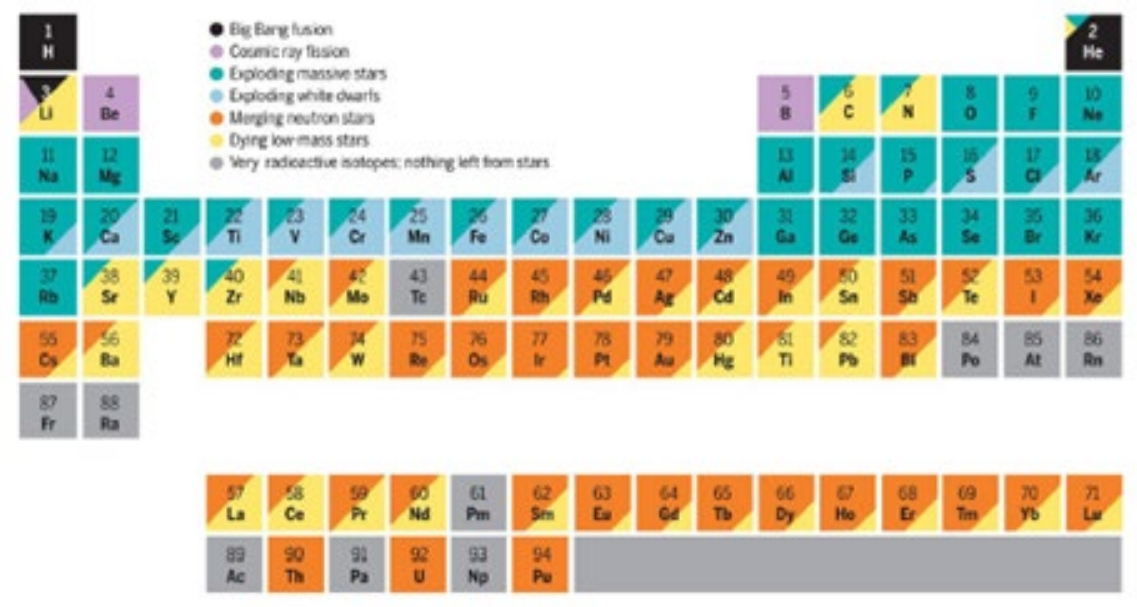




\section{Figure 6}
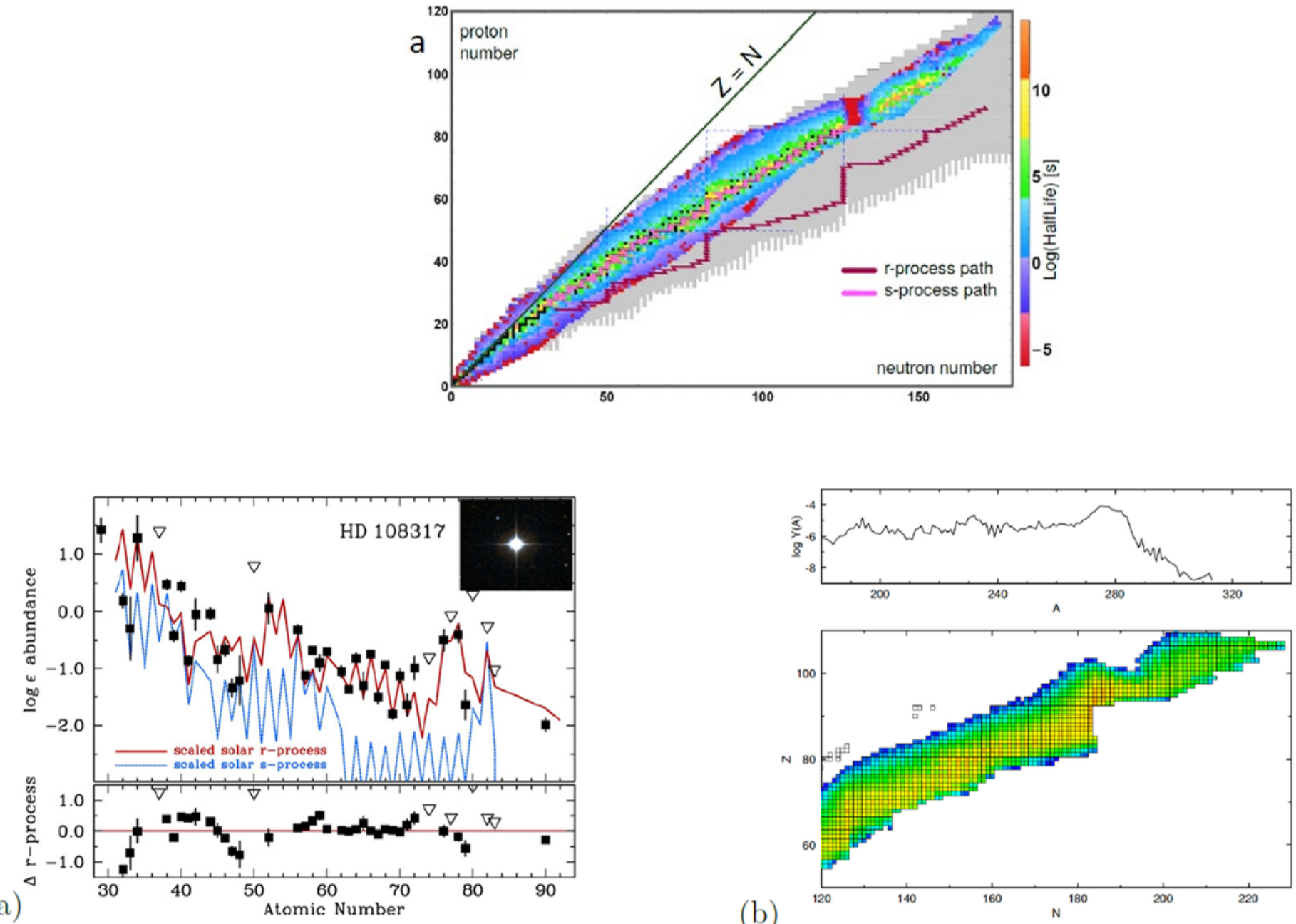

(b)

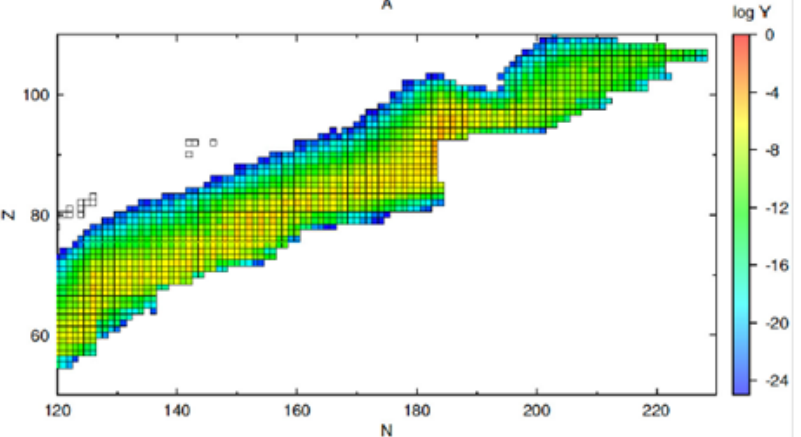




\section{Figure 7}
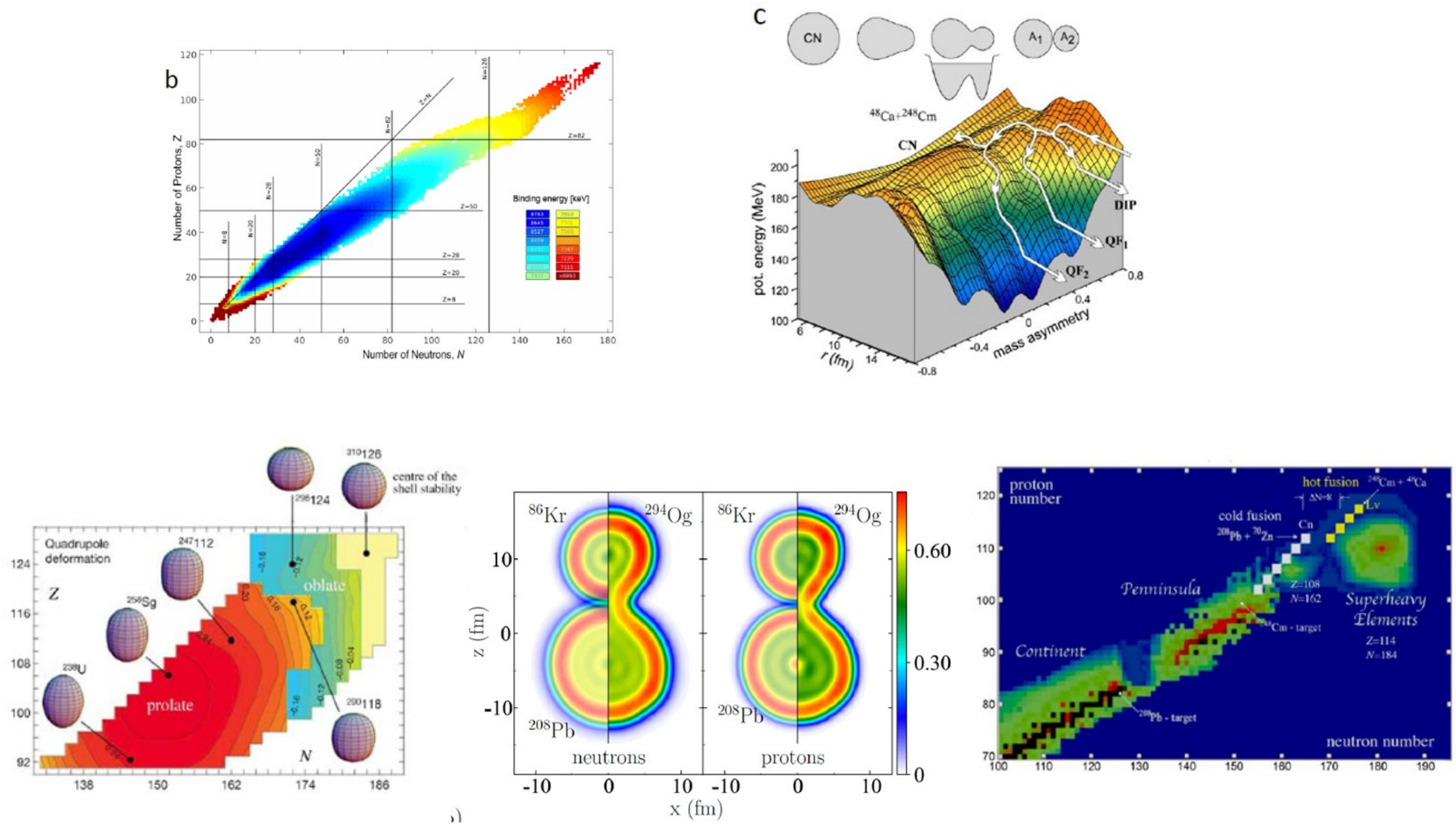


\section{Figure 8}

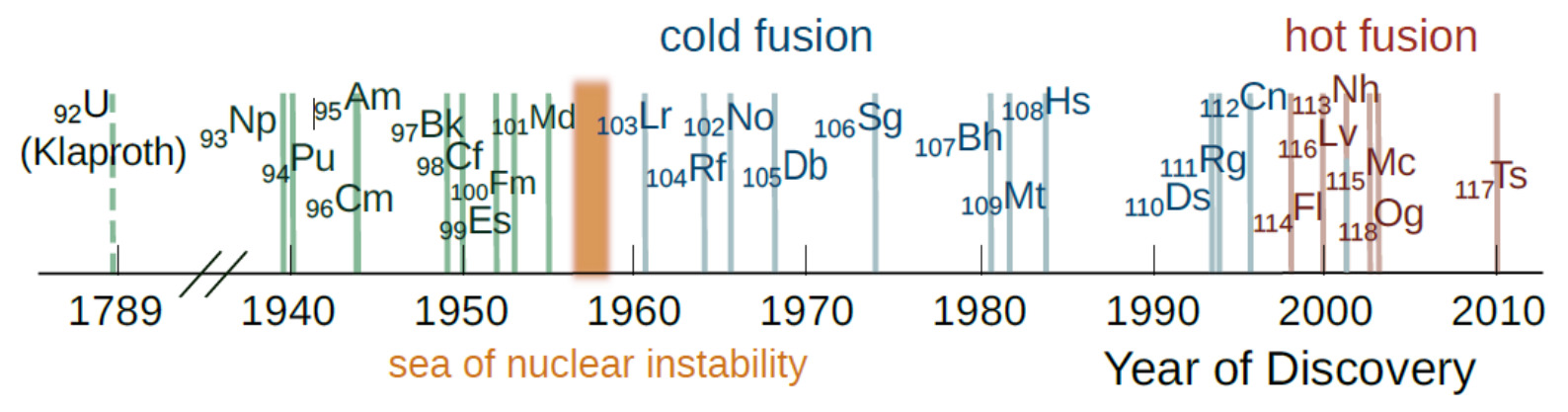

